\title{
Power Consumption Characteristics Research on Mobile System of Electrically Driven Large-Load- Ratio Six-Legged Robot
}

Hong-Chao Zhuang ( $\sim$ zhuanghongchao_hit@163.com )

Tianjin University of Technology and Education https://orcid.org/0000-0003-4091-6171

Ning Wang

Tianjin University of Technology and Education

Hai-Bo Gao

Harbin Institute of Technology

Zong-Quan Deng

Harbin Institute of Technology

Original Article

Keywords: Electrically driven , Large-load-ratio six-legged robot , Power consumption , Mobile system

Posted Date: July 28th, 2021

DOI: https://doi.org/10.21203/rs.3.rs-739083/v1

License: (c) (i) This work is licensed under a Creative Commons Attribution 4.0 International License. Read Full License 


\section{Title page}

\section{Power Consumption Characteristics Research on Mobile System of Electrically Driven Large-Load-Ratio Six-Legged Robot}

Hong-Chao Zhuang, born in 1982, is currently an associate professor and a supervisor for master candidates at School of Mechanical Engineering, Tianjin University of Technology and Education, China. He received his $\mathrm{PhD}$ degree from Harbin Institute of Technology, China, in 2015. His research interests include special robot systems and intelligent robotics.

Tel: +86-22-88181083;

E-mail: zhuanghongchao_hit@163.com

Ning Wang, born in 1981, is currently a lecturer at School of Information Technology Engineering, Tianjin University of Technology and Education, China. She received her PhD degree from Yanshan University, China, in 2015. Her research interests include special robot systems and control of legged robots.

Tel: +86-22-88181059;

E-mail: wangning811108@163.com

Hai-Bo Gao, born in 1970, is currently a professor and a supervisor for PhD candidates at State Key Laboratory of Robotics and System, Harbin Institute of Technology, China. He received his PhD degree from Harbin Institute of Technology, China, in 2004. His research interests include special robot systems and aerospace mechanisms and control.

Tel: +86-451-86402037-801;

E-mail: gaohaibo@hit.edu.cn

Zong-Quan Deng, born in 1956, is currently an Academician of Chinese Academy of Engineering, a professor, and a supervisor for PhD candidates at Harbin Institute of Technology, China. He received his master's degree from Harbin Institute of Technology, China, in 1984. His research interests include planetary rover technology and aerospace mechanisms and control.

Tel: +86-451-86413857;

E-mail: dengzq@hit.edu.cn

Corresponding author: Hong-Chao Zhuang E-mail: zhuanghongchao_hit@163.com 


\title{
Power Consumption Characteristics Research on Mobile System of Electrically Driven Large-Load-Ratio Six-Legged Robot
}

\author{
Hong-Chao Zhuang ${ }^{1}$ Ning Wang ${ }^{2} \cdot$ Hai-Bo Gao ${ }^{3}$ Zong-Quan Deng ${ }^{3}$
}

Received July 18, 2021; revised xx xx, 2021; accepted xx xx, 2021

(C) Chinese Mechanical Engineering Society and Springer-Verlag Berlin Heidelberg 2017

\begin{abstract}
To research the power consumption characteristics of mobile system of an electrically driven large-load-ratio six-legged robot with engineering capability is beneficial to speed up it toward practicability. Based on the configuration and walking modes of robot, the mathematical model of the power consumption of mobile system is set up. In view of the tripod gait is often selected for the six-legged robots, the simplified power consumption model of mobile system under the tripod gait is established by means of reducing the dimension of the robot's statically indeterminate problem and constructing the equal force distribution. Then, the power consumption of robot mobile system is solved under different working conditions. The variable tendencies of the power consumption of robot mobile system are respectively obtained with changes in the rotational angles of hip joint and knee joint, body height, and span. The articulated rotational zones and the ranges of body height and span are determined under the lowest power consumption. According to the walking experiments of prototype, the variable tendencies of the average power consumption of robot mobile system are respectively acquired with changes in the duty ratio, body height, and span. Then, the feasibility and correctness of theory analysis are verified in the power consumption of robot mobile system. The proposed analysis method in this paper can provide a reference on the lower power research of the large-load-ratio multi-legged robots.
\end{abstract}

Keywords: Electrically driven - Large-load-ratio six-legged

\footnotetext{
Hong-Chao Zhuang

zhuanghongchao_hit@163.com
}

1 School of Mechanical Engineering, Tianjin University of Technology and Education, Tianjin 300222, China

2 School of Information Technology Engineering, Tianjin University of Technology and Education, Tianjin 300222, China

3 State Key Laboratory of Robotics and System, Harbin Institute of Technology, Harbin 150001, China robot $\bullet$ Power consumption $\bullet$ Mobile system

\section{Introduction}

With the development of robotics, a large number of autonomous mobile robots have been widely applied [1]. Intelligentization [2], high terrain trafficability [3], and large load ratio [4] are generally regarded as the most important developments for the ground mobile robots. According to the characteristics of mobile mechanisms, most ground mobile robots can be are classified into five categories: wheeled robots, tracked robots, snake robots, legged robots, and wheel-legged robots. Generally, although the wheeled and tracked robots have high mobile speed, their trafficability is low to traverse great rugged terrain. The snake robots have high terrain adaptability, but they usually lack certain bearing capacities relative to other types of robots. The legged robots can flexibly walk on the rugged terrain due to a series of discrete footholds, but their mobile systems often have high power consumption because of many driving joints. Although the wheel-legged robots integrate many capacities from the wheeled robots and the legged robots, they usually have large size, high mass and high power consumption. In view of the excellent terrain trafficability and load capacity, the legged robots usually attract the interest of many researchers.

Load ratio is the ratio of the material load carried by the legged robot to the weight of robot. The high load ratio indicates that the legged robot has the high capacity to carry the goods and materials on its body. According to the load capacity of robot, the legged robots can be classified into two categories: small (non heavy-duty) legged robots and large-load-ratio (heavy-duty) legged robots. Based on the technical parameters of BigDog [5], Ambler [6], 
ATHLETE [7], Dante II [8], and so on, Zhuang et al. [9-11] concluded that the main characteristics of the large-load-ratio legged robots roughly included the larger body mass, bigger body size and large load ratio than the conventional legged robots. The independent energy supply units necessarily need to be carried when the working environment of large-load-ratio legged robots is changed from the laboratory to the outdoor. However, the excessive power consumption of robot system is inconsistent with the insufficient storage capacity of energy devices. Hence, to reduce the power consumption of legged robots is an important means to improve their outdoor survivability, especially for large-load-ratio legged robots. Many scholars, such as Silva [12-14], Sanz-Merodio [15], Roy [16], and so on, all have pointed out that optimizing power consumption is an important problem for the legged robots.

The legged robots can walk through the periodic alternation between the support phase and the transfer phase [17-19]. No matter what kinds of gaits are employed by the legged robots, the swinging legs are mainly used to change the landing points and do not make direct contributions for the walking of legged robots. Although the foot trajectories of swinging legs can be any curves, for example rectangular curve [20] and arc curve [21], there is a requirement that the impact phenomenon do not exist between the feet of legged robots and the ground. Otherwise, the foot velocity is not zero at the moment of landing. And the impact force will consume the system energy of robot. Therefore, both the kinematic and dynamic boundary conditions of the swinging legs should be satisfied when the swinging legs are lifted and landed. To reduce the impact phenomenon between feet and ground, Lei et al. [22] used the combined cycloid function to plan the foot trajectories of a quadruped robot. In aspect of reducing the energy loss of robot system, Zhuang et al. [23-26] developed a single leg with three active degrees of freedom and four passive degrees of freedom for a largeload-ratio six-legged robot. The prototype experiments were used to validate the effectiveness of proposed gait planning and the calculation methods of the articulated torque and rotational speed. Liu et al. [27] carried out the static force analysis of foot for an electrically driven heavy-duty six-legged robot under the tripod gait. Vanderborght et al. [28] selected the controllable flexible actuators to achieve the desired trajectories of a biped robot Lucy, which was propitious to cut down the control quantity and power consumption of robot.

Some researchers studied the internal relations between gait parameters and power consumption for the legged robots. Kaneko et al. [29] analyzed the variable tendencies of energy dissipation rate of the mobile system with changes in the step pitch and body height for an articulated quadruped robot. Estremera and Waldron [30] calculated the energy efficiency of an electro-pneumatic driven quadruped robot called KOLT. The energy loss assessment was executed by the experiments of robot. Zielinska [31, 32] discussed the poses of support legs and the relation between link proportions and power consumption when the legged robots linearly walked along the horizontal/ inclined plane. Lin and Song [33] analyzed the relation between walking speeds and power consumption of system for a pantograph quadruped robot. Silva et al. [34] and Jin et al. [35] discussed the connections between gait parameters of a mammal like six-legged robot and its power consumption. Marhefka and Orin [36] measured the energy dissipation rate with changes in the velocity and duty factor for a pantograph six-legged robot. Nishii [37] studied the energy dissipation rate of an articulated six-legged robot when the walking speed and duty factor were changed. Santos et al. [38] not only discussed the articulated power consumption under the uniform walking in the tripod gait, but also analyzed the power consumption of system with change in body height for an imitation insect type six-legged robot. Many researchers mainly study the virtual prototypes or prototypes of small legged robots in the aspects of gait parameters and power consumption. In addition, it is not easy to found related reports about the research of power consumption for the large-load-ratio legged robots.

When the articulated driving devices and actuating devices are determined, some important parameters can be obtained for calculating the power consumption of robot mobile system, for example articulated transmission ratios, transmission efficiencies, torque constants of servo motors, and so on. Actually, the power consumption of robot mainly comes from the mobile system and control system. The power consumption of control system is generally considered as a constant when the control devices have been selected, and it is usually far less than the power consumption of mobile system. Then, the power consumption of robot mobile system is mainly studied in this paper, and it can be divided into two parts: the power consumption of the support phase and the power consumption of the transfer phase. Meanwhile, the power consumption of the support phase is often greater than that of the transfer phase because that the support phase bears the weights of the robot and materials. The power consumption of the transfer phase is mainly caused by the swing of swinging legs. 
In view of the above problems, an electrically driven large-load-ratio six-legged robot is considered as an example in this paper to present a research on the power consumption characteristics of robot mobile system. This paper is divided into nine sections. In section 2, the configuration of robot and walking modes are designed. In section 3, the mathematical expressions of articulated torques in the support phase and transfer phase are derived by the dynamics analysis of robot. In section 4, the mathematical models of the power consumption are established for the single leg and robot mobile system. In section 5, the power consumption of mobile system under the tripod gait is studied in view of the tripod gait as the common gait and fastest gait of the six-legged robots. The dimension reduction is implemented for the statically indeterminate problem of robot. In section 6 , the constraint conditions of stable walking are discussed. In section 7, the simplified mathematical model of power consumption is built under the tripod gait. The numerical solution is performed for the power consumption of robot mobile system. The articulated rotational zones and the ranges of body height and span are respectively determined under the lowest power consumption. In section 8 , the walking experiments of prototype are carried out. The variable tendencies of the average power consumption of robot mobile system are respectively acquired with changes in the duty ratio, body height and span. In the final section, the conclusions and further work are presented.

(a) Abductor joint

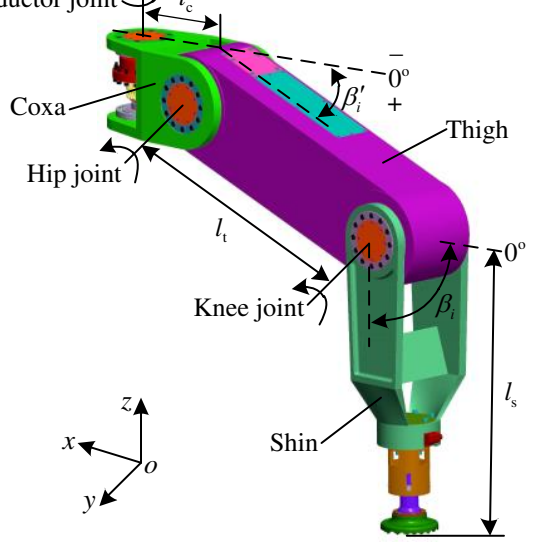

\section{Configuration and Walking Modes of Robot}

\subsection{Configuration of Robot}

In order to facilitate appellation, the electrically driven large-load-ratio six-legged robot is called the large-loadratio six-legged robot for short in this paper. Based on the structure of ambulacra of the hexapod, the single leg of robot is designed, and it includes three joints: an abductor joint, hip joint, and knee joint. Meanwhile, the axis of the abductor joint follows the direction of $z$. The axes of the hip joint and knee joint run parallel to the $y$-axis. $l_{\mathrm{c}}, l_{\mathrm{t}}$, and $l_{\mathrm{s}}$ are respectively regarded as the lengths of coxa, thigh, and shin. The abductor joint, hip joint, and knee joint of leg $i$ are respectively set as $A_{i}, H_{i}$, and $K_{i}$. $\beta_{i}^{\prime}(i=1,2,3,4,5$, $6)$ is defined as the angle between the coxa of leg $i$ and the thigh of leg $i$. The angle $\beta_{i}(i=1,2,3,4,5,6)$ is between the coxa of leg $i$ and the shin of leg $i$.

In order to keep the universal walking characteristics, the configuration of the large-load-six-legged robot is designed as a regular polygon. $s_{u}$ and $t_{r}$ are respectively defined as the legs in the support phase and transfer phase. The sets of the support phase and transfer phase are set as $s=\left(s_{1}, \cdots, s_{k}, \cdots, s_{u}\right)$ and $t=\left(t_{1}, \cdots, t_{e}, \cdots, t_{r}\right)$, respectively. The constraint conditions are $3 \leq u \leq 6$ and $u+r=6$. The structure of single leg and the mechanism of the large-load-ratio six-legged robot are shown in Figure 1 and Figure 2.

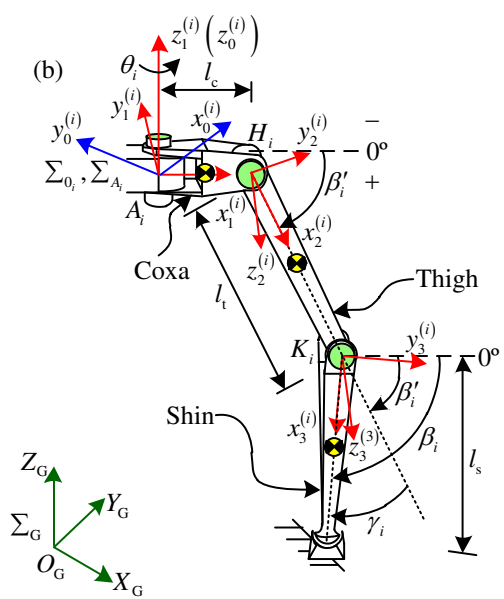

Figure 1 Structure of single leg of large-load-ratio six-legged robot [10, 26, 27] ((a) three-dimensional mod of single leg; (b) coordinate systems of joints in single leg)

In Figure 1, The abductor joint coordinate system of leg $i$ contains $x_{1}^{(i)}, y_{1}^{(i)}$, and $z_{1}^{(i)}$. The hip joint coordinate system of leg $i$ includes $x_{2}^{(i)}, y_{2}^{(i)}$, and $z_{2}^{(i)}$. The knee joint coordinate system of leg $i$ comprises $x_{3}^{(i)}, y_{3}^{(i)}$, and $z_{3}^{(i)}$. In Figure 2, the body coordinate system is set as $\sum_{\mathrm{B}}$, and it is located at the center of body. $\sum_{\mathrm{G}}$ is regarded as the ground coordinate system. The principal vectors and the principal moment are defined by $\boldsymbol{F}_{\mathrm{B}}=\left(F_{\mathrm{B} x}, F_{\mathrm{B} y}, F_{\mathrm{B} z}\right)^{\mathrm{T}}$ 
and $\boldsymbol{M}_{\mathrm{B}}=\left(M_{\mathrm{B} x}, M_{\mathrm{B} y}, M_{\mathrm{B} z}\right)^{\mathrm{T}}$. The $z_{1}^{(i)}$-axis is parallel to the $Z_{\mathrm{B}}$-axis of the body coordinate system. The positive direction of $x_{1}^{(i)}$-axis deviates from the coxa of leg $i$. The leg $i$ can be regarded as lying on the plan $z_{1}^{(i)} x_{1}^{(i)}$. The abductor joint coordinate system $\sum_{A_{i}}$ and foot coordinate system $\sum_{f_{i}}$ of the leg $i$ are in the same positive direction. The positive directions of foot coordinate system $\sum_{o_{i}}$ are parallel to the body coordinate system $\Sigma_{B}$. The foot force of leg $s_{k}$ in the support phase can be divided into ${ }^{\mathrm{B}} F_{x}^{\left(s_{k}\right)}$,

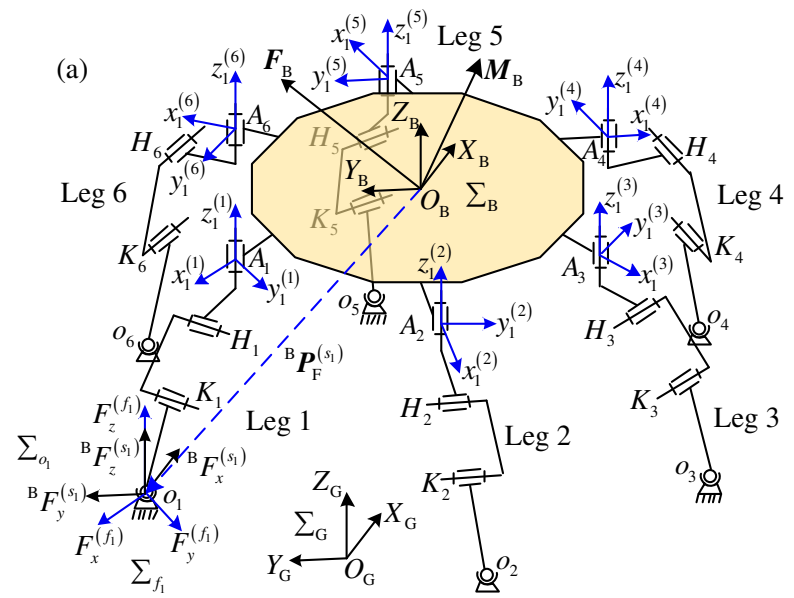

${ }^{\mathrm{B}} F_{y}^{\left(s_{k}\right)}$, and ${ }^{\mathrm{B}} F_{z}^{\left(s_{k}\right)}$ in the coordinate system $\sum_{o_{k}}$, and it can be also divided to $F_{x}^{\left(f_{k}\right)}, F_{y}^{\left(f_{k}\right)}$, and $F_{z}^{\left(f_{k}\right)}$ in the coordinate system $\sum_{f_{k}}$. The position vector of foot end of the leg $s_{k}$ is set as ${ }^{\mathrm{B}} \boldsymbol{P}_{\mathrm{F}}^{\left(s_{k}\right)}$ in the body coordinate system $\Sigma_{\mathrm{B}}$. A coordinate system connecting the leg $i$ and the body is defined as $\Sigma_{0_{i}}$. The coordinate system $\sum_{0_{i}}$ and the abductor joint coordinate system $\sum_{A_{i}}$ will coincide with each other when the rotational angle $\theta_{i}$ of abductor joint is zero degrees for the leg $i$.

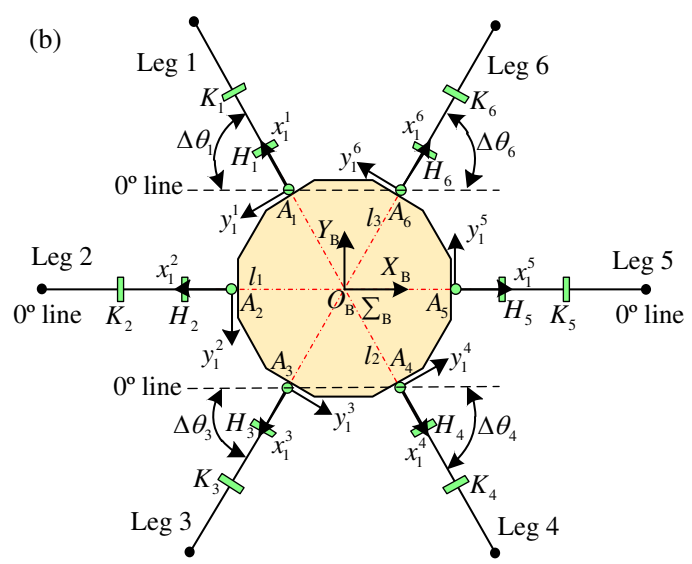

Figure 2 Mechanism and top view of large-load-ratio six-legged robot [10, 26, 27] ((a) mechanism of robot; (b) top view of robot)

In Figure 2, the $0^{\circ}$ line is parallel to the $X_{\mathrm{B}}$ axis of the $\Sigma_{\mathrm{B}}$, and it passes through the corresponding original points of the abductor joint coordinate systems. $\triangle \theta_{i}$ is defined as the initial angle of abductor joint between the $0^{\circ}$ line and the leg $i$, and it is used to set the initial position of abductor joint when the robot begins to walk. The range of $\triangle \theta_{i}$ is from $0^{\circ}$ to $60^{\circ} . \theta_{i}$ is regarded as the rotation angle of abductor joint based on the initial angle $\triangle \theta_{i}$ of leg $i$. The initial angles $\triangle \theta_{2}$ of and $\triangle \theta_{5}$ are always zero for the leg 2 and leg 5 in this paper. The initial angles $\triangle \theta_{1}, \triangle \theta_{3}, \triangle$ $\theta_{4}$, and $\triangle \theta_{6}$ are from $0^{\circ}$ to $60^{\circ}$ for the legs $1,3,4$, and 6 , and they are same when the robot begins to walk. Then, the interference is taken as the evaluation condition of joint rotation amplitude. Based on the range of $\triangle \theta_{i}$, the effective intervals of $\theta_{i}, \beta_{i}{ }^{\prime}$, and $\beta_{i}$ are respectively obtained in the abductor joint coordinate system $\Sigma_{A_{i}}$ of leg $i$ by plotting analysis; they are from $-30^{\circ}$ to $30^{\circ}$ for $\theta_{i}$, from $-90^{\circ}$ to $90^{\circ}$ for $\beta_{i}{ }^{\prime}$, and from $0^{\circ}$ to $150^{\circ}$ for $\beta_{i}$.

As shown in Figure 2, $\Phi_{i}$ is defined as the rotation angle between the $\Sigma_{0_{i}}$ and the $\Sigma_{\mathrm{B}}$ for the leg $i$. The values of the rotation angle $\Phi_{i}$ can be viewed as $120^{\circ}, 180^{\circ}, 240^{\circ}$, $300^{\circ}, 360^{\circ}$, and $60^{\circ}$ for the legs $1,2,3,4,5$, and 6 , respectively. Then, the following equations can be obtained. Then

$$
\begin{aligned}
& { }_{0}^{\mathrm{B}} \boldsymbol{R}^{(i)}=\operatorname{Rot}\left(z, \Phi_{i}\right)=\left(\begin{array}{ccc}
\cos \Phi_{i} & -\sin \Phi_{i} & 0 \\
\sin \Phi_{i} & \cos \Phi_{i} & 0 \\
0 & 0 & 1
\end{array}\right), \\
& { }_{\mathrm{o}}^{\mathrm{f}} \boldsymbol{R}^{(i)}=\left({ }_{0}^{\mathrm{B}} \boldsymbol{R}^{(i)}{ }_{\mathrm{A}}^{0} \boldsymbol{R}^{(i)}\right)^{-1}=\left(\operatorname{Rot}\left(z, \Phi_{i}\right) \operatorname{Rot}\left(z, \theta_{i}\right)\right)^{\mathrm{T}} .
\end{aligned}
$$

where ${ }_{0}^{\mathrm{B}} \boldsymbol{R}^{(i)}$ is the rotation matrix from the $\Sigma_{0_{i}}$ to the $\sum_{\mathrm{B}}$ for the leg $i,{ }_{0}^{\mathrm{B}} \boldsymbol{R}^{(i)} \in \mathbf{R}^{3 \times 3} \cdot{ }_{\mathrm{A}}^{\mathrm{B}} \boldsymbol{R}^{(i)}$ is the rotation matrix from the $\sum_{A_{i}}$ to the $\sum_{\mathrm{B}}$ for the leg $i$, ${ }_{\mathrm{A}}^{\mathrm{B}} \boldsymbol{R}^{(i)} \in \mathbf{R}^{3 \times 3} .{ }_{\mathrm{o}}^{\mathrm{f}} \boldsymbol{R}^{(i)}$ is the rotation matrix from the $\Sigma_{o_{i}}$ to the $\sum_{f_{i}},{ }_{\mathrm{o}}^{\mathrm{f}} \boldsymbol{R}^{(i)} \in \mathbf{R}^{3 \times 3} .{ }_{\mathrm{A}}^{0} \boldsymbol{R}^{(i)}$ is the rotation matrix from the $\sum_{A_{i}}$ to the $\sum_{0_{i}},{ }_{\mathrm{A}}^{0} \boldsymbol{R}^{(i)} \in \mathbf{R}^{3 \times 3}$.

\subsection{Walking Modes of Robot}

Based on the configuration of large-load-ratio six-legged robot, the typical walking modes of robot can be divided 
into the crab type, ant type, mixture type I, and mixture
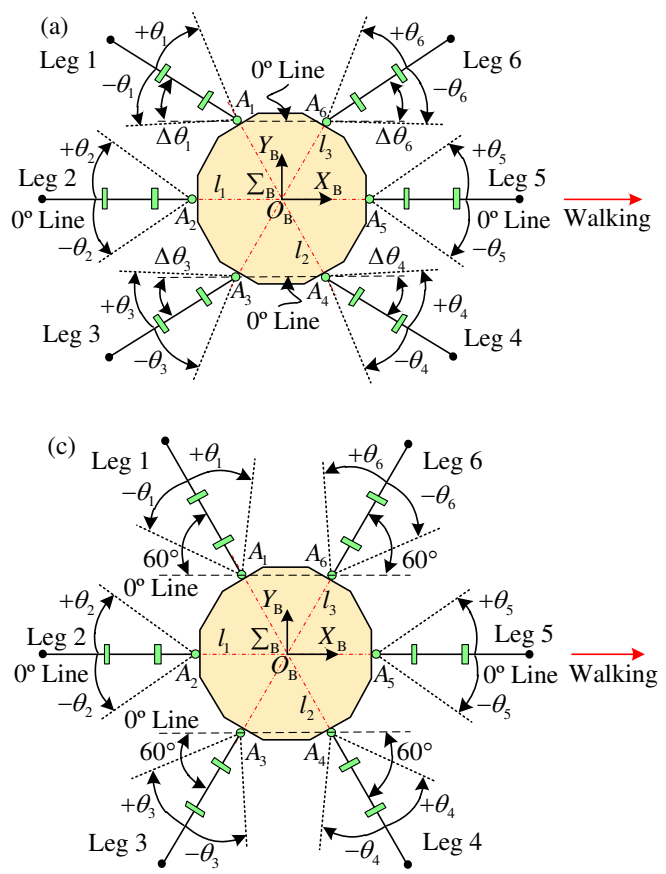

type II, as shown in Figure 3.
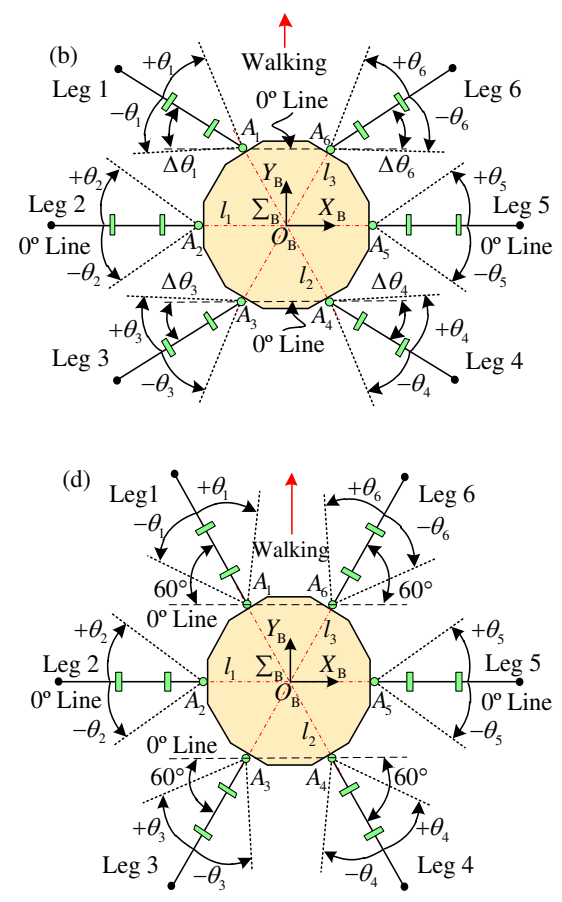

Figure 3 Typical walking modes of large-load-ratio six-legged robot [26] ((a) crab type; (b) ant type; (c) mixed type I; and (d) mixed type II)

The initial attitudes of robot under the crab type and ant type can be arbitrarily set though the initial angles $\Delta \theta_{1}, \Delta \theta_{3}$, $\Delta \theta_{4}$, and $\Delta \theta_{6}$. The crab type and ant type can be changed into the mixed type I and mixed type II, respectively, when the condition is $\Delta \theta_{1}=\Delta \theta_{3}=\Delta \theta_{4}=\Delta \theta_{6}=60^{\circ}$. In Figure $3,+\theta_{i}$ and $-\theta_{i}$ are defined as the front swing angle and rear swing angle based on the $\Delta \theta_{i}$. Hence, $+\theta_{i}$ and $-\theta_{i}$ represent the positive and negative intervals of $\theta_{i}$ respectively. The straight lines $l_{1}, l_{2}$, and $l_{3}$ are respectively orthogonal to the relevant axes of abductor joints.

\section{Dynamics Analysis of Robot}

The walking of large-load-ratio six-legged robot is achieved by periodic alternating between the support phase and the transfer phase. The swinging legs do not refer to the foot forces, and their articulated torques can be obtained by the dynamic equations. The support legs not only support the body of robot and materials, but also need to participate in the walking of robot. Hence, the articulated torques of leg $i$ in the support phase are caused by the movement and foot force of the leg $i$. Meanwhile, the articulated torques from the movement of leg in the support phase can be gained based on the dynamic equations. The articulated torques from the foot forces can be calculated according to the Jacobian matrix and characteristics of series manipulator.

The expression of articulated torque can be written for the leg $i$ of large-load-ratio six-legged robot.

Then

$$
\tilde{M}_{i}=\hat{M}_{i}+\hat{M}_{\mathrm{F} i},
$$

where $\tilde{\boldsymbol{M}}_{i}$ is the vector of articulated torque of the leg $i$, $\tilde{\boldsymbol{M}}_{i} \in \mathbf{R}^{3 \times 1} . \hat{\boldsymbol{M}}_{i}$ is the vector of articulated torque from the dynamics item of leg $i, \hat{\boldsymbol{M}}_{i} \in \mathbf{R}^{3 \times 1}$. $\hat{\boldsymbol{M}}_{\mathrm{F} i}$ is the vector of articulated torque from the foot force of leg $i$, $\hat{\boldsymbol{M}}_{\mathrm{F} i}=\left(\boldsymbol{J}_{i}(\boldsymbol{q})\right)^{\mathrm{T}}{ }^{\mathrm{B}} \boldsymbol{F}_{i} \in \mathbf{R}^{3 \times 1} \cdot\left(\boldsymbol{J}_{i}(\boldsymbol{q})\right)^{\mathrm{T}}$ is the transpose of velocity Jacobian matrix, $\left(\boldsymbol{J}_{i}(\boldsymbol{q})\right)^{\mathrm{T}} \in \mathbf{R}^{3 \times 3} \cdot{ }^{\mathrm{B}} \boldsymbol{F}_{i}$ is the foot force vector of leg $i$ in the coordinate system $\sum_{\mathrm{B}}$ or $\sum_{o_{i}},{ }^{\mathrm{B}} \boldsymbol{F}_{i} \in \mathbf{R}^{3 \times 1}$. The relations are ${ }^{\mathrm{B}} \boldsymbol{F}_{i}={ }^{\mathrm{B}} \boldsymbol{F}_{s_{k}}$ in the support phase of robot and ${ }^{\mathrm{B}} \boldsymbol{F}_{i}={ }^{\mathrm{B}} \boldsymbol{F}_{t_{e}}=0$ in the transfer phase of robot.

Based on the characteristics of Lagrange method, it is employed to perform the dynamic analysis of robot. The Lagrangian equation of articulated torque can be written from the dynamics item of leg $i$. Then 


$$
\hat{\boldsymbol{M}}_{i}=\frac{d}{d t}\left(\frac{\partial L^{(i)}}{\partial \dot{\boldsymbol{q}}}\right)-\frac{\partial L^{(i)}}{\partial \boldsymbol{q}},
$$

where $L^{(i)}$ is the Lagrange function, $L^{(i)}=E^{(i)}-P^{(i)}$. $E^{(i)}$ and $P^{(i)}$ are the kinetic energy and potential energy of leg $i$, respectively.

Due to no $\dot{\boldsymbol{q}}$ in the potential energy, the Equation (4) can be rewritten as follows. Then

$$
\hat{\boldsymbol{M}}_{i}=\frac{d}{d t}\left(\frac{\partial E^{(i)}}{\partial \dot{\boldsymbol{q}}}\right)-\frac{\partial E^{(i)}}{\partial \boldsymbol{q}}+\frac{\partial P^{(i)}}{\partial \boldsymbol{q}},
$$

Based on the solving of the kinetic energy $E^{(i)}$ and potential energy $P^{(i)}$, the matrix of articulated torque from the dynamics item of leg $i$ can be gained as follows. Then

$$
\hat{\boldsymbol{M}}_{i}=\boldsymbol{D}_{i}(\boldsymbol{q}) \ddot{\boldsymbol{q}}+\boldsymbol{h}_{i}(\boldsymbol{q}, \dot{\boldsymbol{q}})+\boldsymbol{g}_{i}(\boldsymbol{q})
$$

where $\boldsymbol{D}_{i}(\boldsymbol{q})$ is the inertial matrix of the joint of leg $i$, $\boldsymbol{D}_{i}(\boldsymbol{q}) \in \mathbf{R}^{3 \times 3} . \boldsymbol{h}_{i}(\boldsymbol{q}, \dot{\boldsymbol{q}})$ is the force vector of leg $i$ including the centrifugal inertial force and Coriolis inertial force, $\boldsymbol{h}_{i}(\boldsymbol{q}, \dot{\boldsymbol{q}}) \in \mathbf{R}^{3 \times 3} . \boldsymbol{g}_{i}(\boldsymbol{q})$ is the gravity vector of leg $i, \boldsymbol{g}_{i}(\boldsymbol{q}) \in \mathbf{R}^{3 \times 1}$. Meanwhile

$$
\begin{aligned}
\boldsymbol{D}_{i}(\boldsymbol{q}) & =\left(\begin{array}{ccc}
\tilde{w} & 0 & 0 \\
0 & m_{\mathrm{t}} l_{\mathrm{t}}^{2} / 4+I_{\mathrm{t}}+m_{\mathrm{s}} l_{\mathrm{t}}^{2} & m_{\mathrm{s}} l_{\mathrm{t}} l_{\mathrm{s}} \cos \gamma_{i} / 2 \\
0 & m_{\mathrm{s}} l_{\mathrm{t}} l_{\mathrm{s}} \cos \gamma_{i} / 2 & \left(m_{\mathrm{s}} l_{\mathrm{s}}^{2}+I_{\mathrm{s}}\right) / 4
\end{array}\right), \\
\boldsymbol{h}_{i}(\boldsymbol{q}, \dot{\boldsymbol{q}}) & =\left(\begin{array}{ccc}
0 & 0 & 0 \\
\hat{w} & 0 & -m_{\mathrm{s}} l_{\mathrm{t}} l_{\mathrm{s}} \dot{\gamma}_{i} \sin \gamma_{i} / 2 \\
\bar{w} & -m_{\mathrm{s}} l_{\mathrm{t}} l_{\mathrm{s}} \dot{\gamma}_{i} \sin \gamma_{i} / 2 & m_{\mathrm{s}} l_{\mathrm{t}} \dot{\beta}_{\mathrm{s}}^{\prime} \sin \gamma_{i} / 2
\end{array}\right),(8) \\
\boldsymbol{g}_{i}(\boldsymbol{q}) & =\left(\begin{array}{lll}
g_{i}(q)_{11} & g_{i}(q)_{21} & \left.g_{i}(q)_{31}\right)^{\mathrm{T}},
\end{array}\right.
\end{aligned}
$$

where

$$
\begin{aligned}
\tilde{w}= & m_{\mathrm{c}} l_{\mathrm{c}}^{2} / 4+I_{\mathrm{c}}+m_{\mathrm{t}}\left(l_{\mathrm{c}}+\frac{1}{2} l_{\mathrm{t}} \cos \beta_{i}^{\prime}\right)^{2} \\
& +m_{\mathrm{s}}\left(l_{\mathrm{c}}+l_{\mathrm{t}} \cos \beta_{i}^{\prime}+\frac{1}{2} l_{\mathrm{s}} \cos \left(\beta_{i}^{\prime}+\gamma_{i}\right)\right)^{2}, \\
\hat{w}= & \frac{1}{2} m_{\mathrm{t}} l_{\mathrm{t}} \dot{\theta}_{i}\left(l_{\mathrm{c}}+\frac{1}{2} l_{\mathrm{t}} \cos \beta_{i}^{\prime}\right) \sin \beta_{i}^{\prime} \\
& +m_{\mathrm{s}} \dot{\theta}_{i}\left(l_{\mathrm{c}}+l_{\mathrm{t}} \cos \beta_{i}^{\prime}+\frac{1}{2} l_{\mathrm{s}} \cos \left(\beta_{i}^{\prime}+\gamma_{i}\right)\right), \\
& \times\left(l_{\mathrm{t}} \sin \beta_{i}^{\prime}+\frac{1}{2} l_{\mathrm{s}} \sin \left(\beta_{i}^{\prime}+\gamma_{i}\right)\right)
\end{aligned}
$$

$\bar{w}=\frac{1}{2} m_{\mathrm{s}} l_{\mathrm{s}} \dot{\theta}_{i}\left(l_{\mathrm{c}}+l_{\mathrm{t}} \cos \beta_{i}^{\prime}+\frac{1}{2} l_{\mathrm{s}} \cos \left(\beta_{i}^{\prime}+\gamma_{i}\right)\right) \times \sin \left(\beta_{i}^{\prime}+\gamma_{i}\right)$,

$g_{i}(q)_{11}=0$,

$g_{i}(q)_{21}=-\frac{m_{\mathrm{t}} g l_{\mathrm{t}} \cos \beta_{i}^{\prime}}{2}-m_{\mathrm{s}} g l_{\mathrm{t}} \cos \beta_{i}^{\prime}-\frac{m_{\mathrm{s}} g l_{\mathrm{s}} \cos \left(\beta_{i}^{\prime}+\gamma_{i}\right)}{2}$,

$g_{i}(q)_{31}=-\frac{1}{2} m_{\mathrm{s}} g l_{\mathrm{s}} \cos \left(\beta_{i}^{\prime}+\gamma_{i}\right)$.

Then, the dynamic equations of the support leg and swinging leg can be acquired based on Equations (3) and (6), respectively. Generally, the maximum walking speed is not much heigh for the large-load-ratio six-legged robot. The moving speed of robot is often less than the maximum walking speed. Therefore, $\ddot{\boldsymbol{q}}$ and $\dot{\boldsymbol{q}}$ can be approximately ignored in the dynamic analysis. The gravity vector term is mainly considered.

To improve the stability of the large-load-ratio six-legged robot, the bottom of body keeps parallel to the ground when the robot walks. $h$ is defined as the body height of robot from the coordinate system $\sum_{A_{i}}$ to the ground. In view of the foot force in the support phase of robot, the articulated torques of the support leg $s_{k}$ can be respectively gained for the torque $M_{\mathrm{SA}}^{\left(s_{k}\right)}$ of abductor joint, torque $M_{\mathrm{SH}}^{\left(s_{k}\right)}$ of hip joint, and torque $M_{\mathrm{SK}}^{\left(s_{k}\right)}$ of knee joint. Then

$$
\left(\begin{array}{l}
M_{\mathrm{SA}}^{\left(s_{k}\right)} \\
M_{\mathrm{SH}}^{\left(s_{k}\right)} \\
M_{\mathrm{SK}}^{\left(s_{k}\right)}
\end{array}\right)=\left(\begin{array}{ccc}
0 & R_{12} & 0 \\
R_{21} & 0 & R_{23} \\
l_{\mathrm{s}} \sin \beta_{k} & 0 & l_{\mathrm{s}} \cos \beta_{k}
\end{array}\right)\left(\begin{array}{c}
F_{x}^{\left(f_{k}\right)} \\
F_{y}^{\left(f_{k}\right)} \\
F_{z}^{\left(f_{k}\right)}
\end{array}\right),
$$

where

$$
\begin{aligned}
& R_{12}=l_{\mathrm{c}}+l_{\mathrm{t}} \cos \beta_{k}^{\prime}+l_{\mathrm{s}} \cos \beta_{k}, \\
& R_{21}=l_{\mathrm{t}} \sin \beta_{k}^{\prime}+l_{\mathrm{s}} \sin \beta_{k}, \\
& R_{23}=l_{\mathrm{t}} \cos \beta_{k}^{\prime}+l_{\mathrm{s}} \cos \beta_{k},
\end{aligned}
$$

In view of the movement of robot in the support phase, the articulated torques of the support leg $s_{k}$ can be respectively obtained for the torque $M_{\mathrm{DA}}^{\left(s_{k}\right)}$ of abductor joint, torque $M_{\mathrm{DH}}^{\left(s_{k}\right)}$ of hip joint, and torque $M_{\mathrm{DK}}^{\left(s_{k}\right)}$ of knee joint. Then

$$
\left(\begin{array}{l}
M_{\mathrm{DA}}^{\left(s_{k}\right)} \\
M_{\mathrm{DH}}^{\left(s_{k}\right)} \\
M_{\mathrm{DK}}^{\left(s_{k}\right)}
\end{array}\right)=\left(\begin{array}{c}
0 \\
-\frac{1}{2} m_{\mathrm{t}} g l_{\mathrm{t}} \cos \beta_{k}^{\prime}-m_{\mathrm{s}} g l_{\mathrm{t}} \cos \beta_{k}^{\prime}-\frac{1}{2} m_{\mathrm{s}} g l_{\mathrm{s}} \cos \beta_{k} \\
-m_{\mathrm{s}} g l_{\mathrm{s}} \cos \beta_{k} / 2
\end{array}\right),
$$


Based on Equations (3), (10), and (11), the total articulated torques of the support leg $s_{k}$ can be respectively acquired for the torque $M_{\mathrm{A}}^{\left(s_{k}\right)}$ of abductor joint, torque $M_{\mathrm{H}}^{\left(s_{k}\right)}$ of hip joint, and torque $M_{\mathrm{K}}^{\left(s_{k}\right)}$ of knee joint.

Then

$$
\left\{\begin{aligned}
M_{\mathrm{A}}^{\left(s_{k}\right)}= & F_{y}^{\left(f_{k}\right)}\left(l_{\mathrm{c}}+l_{\mathrm{t}} \cos \beta_{k}^{\prime}+l_{\mathrm{s}} \cos \beta_{k}\right) \\
M_{\mathrm{H}}^{\left(s_{\mathrm{k}}\right)}= & F_{x}^{\left(f_{k}\right)}\left(l_{\mathrm{t}} \sin \beta_{k}^{\prime}+l_{\mathrm{s}} \sin \beta_{k}\right) \\
& +\left(F_{z}^{\left(f_{k}\right)}-m_{\mathrm{t}} g / 2-m_{\mathrm{s}} g\right) l_{\mathrm{t}} \cos \beta_{k}^{\prime} \\
& +\left(F_{z}^{\left(f_{k}\right)}-m_{\mathrm{s}} g / 2\right) l_{\mathrm{s}} \cos \beta_{k} \\
M_{\mathrm{K}}^{\left(s_{k}\right)}= & F_{x}^{\left(f_{k}\right)} l_{\mathrm{s}} \sin \beta_{k}+\left(F_{z}^{\left(f_{k}\right)}-m_{\mathrm{s}} g / 2\right) l_{\mathrm{s}} \cos \beta_{k}
\end{aligned}\right.
$$

Based on Equation (5), the articulated torques of the swinging leg $t_{e}$ can be respectively gained for the torque $M_{\mathrm{A}}^{\left(t_{e}\right)}$ of abductor joint, the torque $M_{\mathrm{H}}^{\left(t_{e}\right)}$ of hip joint, and the torque $M_{\mathrm{K}}^{\left(t_{e}\right)}$ of knee joint.

Then

$$
\begin{aligned}
& \left(\begin{array}{lll}
M_{\mathrm{A}}^{\left(t_{e}\right)} & M_{\mathrm{H}}^{\left(t_{e}\right)} & M_{\mathrm{K}}^{\left(t_{e}\right)}
\end{array}\right)^{\mathrm{T}}=\left(\begin{array}{ccc}
M_{\mathrm{DA}}^{\left(t_{e}\right)} & M_{\mathrm{DH}}^{\left(t_{e}\right)} & M_{\mathrm{DK}}^{\left(t_{e}\right)}
\end{array}\right)^{\mathrm{T}} \\
& 0 \\
& =\left(\begin{array}{c}
-m_{\mathrm{t}} g l_{\mathrm{t}} \cos \beta_{e}^{\prime} / 2-m_{\mathrm{s}} g l_{\mathrm{t}} \cos \beta_{e}^{\prime}-m_{\mathrm{s}} g l_{\mathrm{s}} \cos \beta_{e} / 2 \\
-m_{\mathrm{s}} g l_{\mathrm{s}} \cos \beta_{e} / 2
\end{array}\right) .
\end{aligned}
$$

\section{Power Consumption Model of Robot}

The power consumption of robot mobile system mainly comes from the electronic control devices of robot control system and the articulated driving and actuating devices of legs. Meanwhile, the latter occupies the main part of power consumption. The effective power consumption of robot mobile system is the basis for walking smoothly and accomplishing the assigned tasks. In this paper, the effective power consumption of robot mobile system is studied. Actually, the driven torques of joints directly determine the power consumption of robot mobile system.

\subsection{Power Consumption Model of Single Leg}

The driving devices of joints are the direct current servo motors for the large-load-ratio six-legged robot. The actuating devices of joints are composed of the worm gears and harmonic reducers. According to the characteristics of servo motor, the final output torques of leg $i$ can be calculated for the torque $M_{\mathrm{A}}^{(i)}$ of abductor joint, the torque $M_{\mathrm{H}}^{(i)}$ of hip joint, and the torque $M_{\mathrm{K}}^{(i)}$ of knee joint. Then

$$
\left(\begin{array}{c}
M_{\mathrm{A}}^{(i)} \\
M_{\mathrm{H}}^{(i)} \\
M_{\mathrm{K}}^{(i)}
\end{array}\right)=\left(\begin{array}{c}
K_{\mathrm{MA}}^{(i)} i_{\mathrm{CA}}^{(i)} i_{\mathrm{A}}^{(i)} \eta_{\mathrm{A}}^{(i)} \\
K_{\mathrm{MH}}^{(i)} i_{\mathrm{CH}}^{(i)} i_{\mathrm{H}}^{(i)} \eta_{\mathrm{H}}^{(i)} \\
K_{\mathrm{MK}}^{(i)} i_{\mathrm{CK}}^{(i)} i_{\mathrm{K}}^{(i)} \eta_{\mathrm{K}}^{(i)}
\end{array}\right),
$$

where $K_{\mathrm{MA}}^{(i)}, K_{\mathrm{MH}}^{(i)}$, and $K_{\mathrm{MK}}^{(i)}$ are the torque constants of servo motors for the abductor joint, hip joint, and knee joint of leg $i . \quad i_{\mathrm{CA}}^{(i)}, \quad i_{\mathrm{CH}}^{(i)}$, and $i_{\mathrm{CK}}^{(i)}$ are the currents of servo motors for the abductor joint, hip joint, and knee joint of leg i. $i_{\mathrm{A}}^{(i)}, i_{\mathrm{H}}^{(i)}$, and $i_{\mathrm{K}}^{(i)}$ are the transmission ratios for the abductor joint, hip joint, and knee joint of leg $i . \eta_{\mathrm{A}}^{(i)}, \eta_{\mathrm{H}}^{(i)}$, and $\eta_{\mathrm{K}}^{(i)}$ are the transmission efficiencies for the abductor joint, hip joint, and knee joint of leg $i$.

No matter whether the servo motors make the positive or negative work, the power consumption of every servo motor can be expressed by the loaded voltage and its own current. Then, the power consumption of servo motors can be respectively written for the abductor joint, hip joint, and knee joint of leg $i$. Then

$$
\left(\begin{array}{c}
P_{\mathrm{A}}^{(i)} \\
P_{\mathrm{H}}^{(i)} \\
P_{\mathrm{K}}^{(i)}
\end{array}\right)=\left(\begin{array}{l}
\left|U_{\mathrm{A}}^{(i)} i_{\mathrm{CA}}^{(i)}\right| \\
\left|U_{\mathrm{H}}^{(i)} i_{\mathrm{CH}}^{(i)}\right| \\
\left|U_{\mathrm{K}}^{(i)} i_{\mathrm{CK}}^{(i)}\right|
\end{array}\right),
$$

where $P_{\mathrm{A}}^{(i)}, P_{\mathrm{H}}^{(i)}$, and $P_{\mathrm{K}}^{(i)}$ are the power consumption of servo motors for the abductor joint, hip joint, and knee joint of leg i. $U_{\mathrm{A}}^{(i)}, U_{\mathrm{H}}^{(i)}$, and $U_{\mathrm{K}}^{(i)}$ are the voltages of servo motors for the abductor joint, hip joint, and knee joint of leg $i$.

Based on Equation (14), the currents of servo motors can be gained. The corresponding currents are brought into Equation (15). The Equation (15) can be rewritten as follows. Then

$$
\left(\begin{array}{c}
P_{\mathrm{A}}^{(i)} \\
P_{\mathrm{H}}^{(i)} \\
P_{\mathrm{K}}^{(i)}
\end{array}\right)=\left(\begin{array}{l}
\left|U_{\mathrm{A}}^{(i)} M_{\mathrm{A}}^{(i)}\right| / K_{\mathrm{MA}}^{(i)} i_{\mathrm{A}}^{(i)} \eta_{\mathrm{A}}^{(i)} \\
\left|U_{\mathrm{H}}^{(i)} M_{\mathrm{H}}^{(i)}\right| / K_{\mathrm{MH}}^{(i)} i_{\mathrm{H}}^{(i)} \eta_{\mathrm{H}}^{(i)} \\
\left|U_{\mathrm{K}}^{(i)} M_{\mathrm{K}}^{(i)}\right| / K_{\mathrm{MK}}^{(i)} i_{\mathrm{K}}^{(i)} \eta_{\mathrm{K}}^{(i)}
\end{array}\right),
$$

Actually, when the specific models of the driving devices and actuating devices of joints are determined, the torque constants, transmission ratios, and transmission efficiencies in Equation (16) can be also obtained for the servo motors. The homogeneous joint of large-load-ratio six-legged robot has the same driving device and actuating device in the abductor joints, hip joints, and knee joints. The performance parameters of joints of the leg $i$ are 
shown in Table 1.

Table 1 Performance parameters of driving devices and actuating devices of joints

\begin{tabular}{lllll}
\hline & Rated & Torque & Transmission & $\begin{array}{l}\text { Transmission } \\
\text { Joint }\end{array}$ \\
$\begin{array}{llll}\text { voltage } \\
U^{(i)} /(\mathrm{V})\end{array}$ & $\begin{array}{c}K_{\mathrm{M}}^{(i)} /(\mathrm{mN} \cdot \mathrm{m}) \\
i^{(i)} /(\mathrm{mm})\end{array}$ & $\eta^{(i)}$ \\
\hline $\begin{array}{l}\text { Abductor } \\
\text { joint }\end{array}$ & 48 & 40.6 & 2240 & 0.455 \\
Hip joint & 48 & 84.9 & 2220 & 0.455 \\
Knee joint & 48 & 40.6 & 4640 & 0.455 \\
\hline
\end{tabular}

Based on Equation (16), the mathematical model of power consumption $P_{\text {sum }}^{(i)}$ can be established for the leg $i$. Then

$$
P_{\mathrm{sum}}^{(i)}=P_{\mathrm{A}}^{(i)}+P_{\mathrm{H}}^{(i)}+P_{\mathrm{K}}^{(i)}
$$

\subsection{Power Consumption Model of Robot Mobile System}

To easily analyze the power consumption of robot mobile system, it is assumed that the robot does not have any instantaneous random perturbations in the process of walking, or the influences of instantaneous random perturbations can be approximately ignored on the mobile system of robot. Based on Equation (17), the mathematical model of the power consumption $P_{\text {sum }}$ of robot mobile system can be expressed as follows.

Then

$$
P_{\mathrm{sum}}=\sum_{i=1}^{6}\left(P_{\mathrm{A}}^{(i)}+P_{\mathrm{H}}^{(i)}+P_{\mathrm{K}}^{(i)}\right),
$$

According to the characteristics of servo motor, it can be obtained that the final output torque of servo motor directly depends on the load torque of joint. When the number of support legs and number of swinging legs are $u$ and $r$ respectively and the conditions are $u+r=6,3 \leq u \leq 6$, and $0 \leq r \leq 3$, Equation (18) can be rewritten as follows. Then

$$
\begin{aligned}
P_{\text {sum }}= & \sum_{k}^{u}\left(\frac{\left|U_{\mathrm{A}}^{\left(s_{k}\right)} M_{\mathrm{A}}^{\left(s_{k}\right)}\right|}{K_{\mathrm{MA}}^{\left(s_{k}\right)} i_{\mathrm{A}}^{\left(s_{k}\right)} \eta_{\mathrm{A}}^{\left(s_{k}\right)}}+\frac{\left|U_{\mathrm{H}}^{\left(s_{k}\right)} M_{\mathrm{H}}^{\left(s_{k}\right)}\right|}{K_{\mathrm{MH}}^{\left(s_{k}\right)} i_{\mathrm{H}}^{\left(s_{k}\right)} \eta_{\mathrm{H}}^{\left(s_{k}\right)}}+\frac{\left|U_{\mathrm{K}}^{\left(s_{k}\right)} M_{\mathrm{K}}^{\left(s_{k}\right)}\right|}{K_{\mathrm{MK}}^{\left(s_{k}\right)} i_{\mathrm{K}}^{\left(s_{k}\right)} \eta_{\mathrm{K}}^{\left(s_{k}\right)}}\right) \\
& +\sum_{e}^{r}\left(\frac{\left|U_{\mathrm{A}}^{\left(t_{e}\right)} M_{\mathrm{A}}^{\left(t_{e}\right)}\right|}{K_{\mathrm{MA}}^{\left(t_{e}\right)} i_{\mathrm{A}}^{\left(t_{e}\right)} \eta_{\mathrm{A}}^{\left(t_{e}\right)}}+\frac{\left|U_{\mathrm{H}}^{\left(t_{e}\right)} M_{\mathrm{H}}^{\left(t_{e}\right)}\right|}{K_{\mathrm{MH}}^{\left(t_{e}\right)} i_{\mathrm{H}}^{\left(t_{e}\right)} \eta_{\mathrm{H}}^{\left(t_{e}\right)}}+\frac{\left|U_{\mathrm{K}}^{\left(t_{e}\right)} M_{\mathrm{K}}^{\left(t_{e}\right)}\right|}{K_{\mathrm{MK}}^{\left(t_{e}\right)} i_{\mathrm{K}}^{\left(t_{e}\right)} \eta_{\mathrm{K}}^{\left(t_{e}\right)}}\right),
\end{aligned}
$$

Due to the consistent of started state or stopped state for the joint rotation, the same rated voltages $U^{(i)}$ of servo motors and low walking speed of robot, the supply voltage of servo motor can be approximated as a constant term of $P_{\text {sum }}$ based on Equation (19), although the articulated rotating speed affects the input voltage of servo motor. It is assumed that the servo motor operates near the rated voltage. Then, the final output torque of joint is variable in Equation (19). Equation (12), Equation (13) and the data in Table 1 are brought into Equation (19). The final expression of mathematical model can be obtained for the power consumption of robot mobile system. Then

$$
\begin{aligned}
P_{\text {sum }}= & \sum_{k}^{u}\left(1.16\left|M_{\mathrm{A}}^{\left(s_{k}\right)}\right|+0.56\left(\left|M_{\mathrm{H}}^{\left(s_{k}\right)}\right|+\left|M_{\mathrm{K}}^{\left(s_{k}\right)}\right|\right)\right)+\sum_{e}^{r}\left(1.16\left|M_{\mathrm{A}}^{\left(t_{e}\right)}\right|+0.56\left(\left|M_{\mathrm{H}}^{\left(t_{e}\right)}\right|+\left|M_{\mathrm{K}}^{\left(t_{e}\right)}\right|\right)\right) \\
= & \sum_{k}^{u}\left(\left|1.16 F_{y}^{\left(f_{k}\right)}\left(l_{\mathrm{c}}+l_{\mathrm{t}} \cos \beta_{k}^{\prime}+l_{\mathrm{s}} \cos \beta_{k}\right)\right|+\mid 0.56 F_{x}^{\left(f_{k}\right)}\left(l_{\mathrm{t}} \sin \beta_{k}^{\prime}+l_{\mathrm{s}} \sin \beta_{k}\right)+\left(0.56 F_{z}^{\left(f_{k}\right)}-0.28 m_{\mathrm{t}} g-0.56 m_{\mathrm{s}} g\right)\right. \\
& \left.\quad \times l_{\mathrm{t}} \cos \beta_{k}^{\prime}+\left(0.56 F_{z}^{\left(f_{k}\right)}-0.28 m_{\mathrm{s}} g\right) l_{\mathrm{s}} \cos \beta_{k}|+| 0.56 F_{x}^{\left(f_{k}\right)} l_{\mathrm{s}} \sin \beta_{k}+\left(0.56 F_{z}^{\left(f_{k}\right)}-0.28 m_{\mathrm{s}} g\right) \times l_{\mathrm{s}} \cos \beta_{k} \mid\right) \\
& +\sum_{e}^{r}\left(\left|0.28 m_{\mathrm{t}} g l_{\mathrm{t}} \cos \beta_{e}^{\prime}+0.56 m_{\mathrm{s}} g l_{\mathrm{t}} \cos \beta_{e}^{\prime}+0.28 m_{\mathrm{s}} g l_{\mathrm{s}} \cos \beta_{e}\right|+\left|0.28 m_{\mathrm{s}} g l_{\mathrm{s}} \cos \beta_{e}\right|\right)
\end{aligned}
$$

To effectively reduce the power consumption of robot mobile system is a guarantee on prolonging the working time of the large-load-ratio six-legged robot. The minimum power consumption of robot mobile system can be regarded as a target. Then

$$
\min _{F_{x}^{\left(f_{k}\right)}, F_{y}^{\left(f_{k}\right)}, F_{z}^{\left(f_{k}\right)}, \beta_{k}^{\prime}, \beta_{k}, \beta_{e}^{\prime}, \beta_{e}} P_{\text {sum }}
$$

Based on Equations (20) and (21), it can be obtained that to calculate the minimum power consumption of robot mobile system is translated to determine the minimum foot force under the poses of robot. Owing to the many variables in Equation (21), it is difficult to directly solve the minimum power consumption of robot mobile system. Therefore, the power consumption analysis of robot mobile system can be implemented through simplifying Equation (20). 
In Equation (20), the foot force of leg $i$ lies in the coordinate system $\sum_{f_{i}}$ of foot end. To facilitate the analysis of power consumption, the foot force $\boldsymbol{F}^{\left(f_{i}\right)}$ of $\operatorname{leg} i$ is converted to the coordinate system $\sum_{o_{i}}$ of foot end. The top view of foot coordinate system and pose of single leg $i$ are shown in Figure 4.
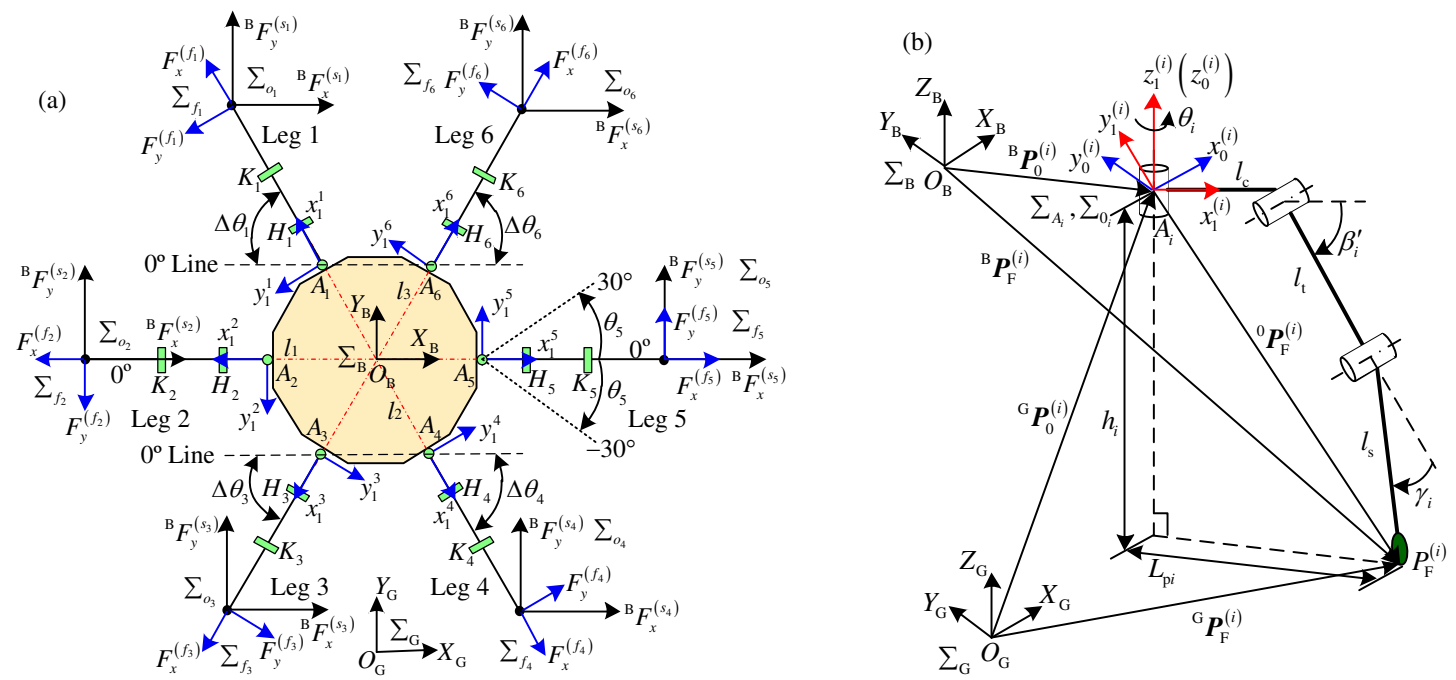

Figure 4 Top view of foot coordinate system and pose of single leg $i$ [26] ((a) foot coordinate system; (b) pose of single leg $i$ )

In Figure 4, $s$ is regarded as the step pitch of robot. The span $L_{\mathrm{p} i}$ is the projection length of leg $i$ in the coordinate system of abductor joint. $L_{\mathrm{p} i}^{\prime}$ represents the initial span of leg $i$ when the robot is about to walk. $h_{i}$ is defined as the distance from the coordinate origin of the $\sum_{A_{i}}$ to the ground. It can be obtained that $h_{i}$ is equal to the body height $h$ of robot. ${ }^{\mathrm{G}} \boldsymbol{P}_{\mathrm{F}}^{(i)}$ is the position matrix from the foot of leg $i$ to the ground coordinate system $\sum_{\mathrm{G}} \cdot{ }^{\mathrm{G}} \boldsymbol{P}_{0}^{(i)}$ represents the position matrix from the coordinate system $\sum_{0_{i}}$ to the ground coordinate system $\sum_{\mathrm{G}} \cdot{ }^{0} \boldsymbol{P}_{\mathrm{F}}^{(i)}$ is defined as the foot position matrix of leg $i$ in the coordinate system $\sum_{0_{i}} \cdot{ }^{\mathrm{B}} \boldsymbol{P}_{0}^{(i)}$ is regarded as the position matrix from the coordinate system $\Sigma_{0_{i}}$ to the body coordinate system $\sum_{\mathrm{B}}$ for the leg $i$.

Based on Equation (2) and Figure 4, the rotation matrix ${ }_{\mathrm{o}}^{\mathrm{f}} \boldsymbol{R}^{(i)}$ can be obtained for the leg 1 to leg 6 . Then

$$
{ }_{\mathrm{o}}^{\mathrm{f}} \boldsymbol{R}^{(1)}=\left(\begin{array}{ccc}
{ }_{\mathrm{o}}^{\mathrm{f}} R_{11}^{(1)} & { }_{\mathrm{o}}^{\mathrm{f}} R^{(1)} & 0 \\
{ }_{0}^{\mathrm{f}} R^{(1)}{ }_{21} & { }_{\mathrm{o}}^{\mathrm{f}} R^{(1)} & 0 \\
0 & 0 & 1
\end{array}\right),
$$

where

$$
\begin{aligned}
& { }_{\mathrm{o}}^{\mathrm{f}} R^{(1)}{ }_{11}=-0.5 \cos \theta_{1}-0.866 \sin \theta_{1}, \\
& { }_{\mathrm{o}}^{\mathrm{f}} R^{(1)}{ }_{12}=-0.5 \sin \theta_{1}+0.866 \cos \theta_{1}, \\
& { }_{\mathrm{o}}^{\mathrm{f}} R^{(1)}{ }_{21}=0.5 \sin \theta_{1}-0.866 \cos \theta_{1},
\end{aligned}
$$

$$
\begin{aligned}
&{ }_{\mathrm{o}}^{\mathrm{f}} R_{22}^{(1)}=-0.5 \cos \theta_{1}-0.866 \sin \theta_{1}, \\
&{ }_{\mathrm{o}}^{\mathrm{f}} \boldsymbol{R}^{(2)}=\left(\begin{array}{ccc}
-\cos \theta_{2} & -\sin \theta_{2} & 0 \\
\sin \theta_{2} & -\cos \theta_{2} & 0 \\
0 & 0 & 1
\end{array}\right), \\
&{ }_{\mathrm{o}}^{\mathrm{f}} \boldsymbol{R}^{(3)}=\left(\begin{array}{ccc}
{ }_{\mathrm{o}}^{\mathrm{f}} R^{(3)} & { }_{\mathrm{o}}^{\mathrm{f}} R^{(3)} & 0 \\
{ }_{0}^{\mathrm{f}} R^{(3)} & { }_{\mathrm{o}}^{\mathrm{f}} R^{(3)} & 0 \\
0 & 0 & 1
\end{array}\right),
\end{aligned}
$$

where

$$
\begin{gathered}
{ }_{\mathrm{o}}^{\mathrm{f}} R^{(3)}=-0.5 \cos \theta_{3}+0.866 \sin \theta_{3}, \\
{ }_{\mathrm{o}}^{\mathrm{f}} R^{(3)}{ }_{12}=-0.5 \sin \theta_{3}-0.866 \cos \theta_{3}, \\
{ }_{0}^{\mathrm{f}} R^{(3)}{ }_{21}=0.5 \sin \theta_{3}+0.866 \cos \theta_{3}, \\
{ }_{\mathrm{o}}^{\mathrm{f}} R^{(3)}{ }_{22}=-0.5 \cos \theta_{3}+0.866 \sin \theta_{3}, \\
{ }_{\mathrm{o}}^{\mathrm{f}} \boldsymbol{R}^{(4)}=\left(\begin{array}{ccc}
{ }_{0}^{\mathrm{f}} R^{(4)} & { }_{\mathrm{o}}^{\mathrm{f}} R_{12}^{(4)} & 0 \\
{ }_{\mathrm{o}}^{\mathrm{f}} R^{(4)} & { }_{\mathrm{o}}^{\mathrm{f}} R^{(4)} & 0 \\
0 & 0 & 1
\end{array}\right),
\end{gathered}
$$

where

$$
\begin{aligned}
& { }_{\mathrm{o}}^{\mathrm{f}} R_{11}^{(4)}=0.5 \cos \theta_{4}+0.866 \sin \theta_{4}, \\
& { }_{\mathrm{o}}^{\mathrm{f}} R_{12}^{(4)}=0.5 \sin \theta_{4}-0.866 \cos \theta_{4},
\end{aligned}
$$




$$
\begin{aligned}
&{ }_{\mathrm{o}}^{\mathrm{f}} R^{(4)}{ }_{21}=-0.5 \sin \theta_{4}+0.866 \cos \theta_{4}, \\
&{ }_{\mathrm{o}}^{\mathrm{f}} R^{(4)}{ }_{22}= 0.5 \cos \theta_{4}+0.866 \sin \theta_{4}, \\
&{ }_{\mathrm{o}}^{\mathrm{f}} \boldsymbol{R}^{(5)}=\left(\begin{array}{ccc}
\cos \theta_{5} & \sin \theta_{5} & 0 \\
-\sin \theta_{5} & \cos \theta_{5} & 0 \\
0 & 0 & 1
\end{array}\right), \\
&{ }_{\mathrm{f}}^{\mathrm{f}} \boldsymbol{R}^{(6)}=\left(\begin{array}{ccc}
{ }_{\mathrm{o}}^{\mathrm{f}} R^{(6)} & { }_{0}^{\mathrm{f}} R_{12}^{(6)} & 0 \\
{ }_{\mathrm{o}}^{\mathrm{f}} R^{(6)} & { }_{\mathrm{o}}^{\mathrm{f}} R_{22}^{(6)} & 0 \\
0 & 0 & 1
\end{array}\right),
\end{aligned}
$$

where

$$
\begin{aligned}
& { }_{0}^{\mathrm{f}} R_{11}^{(6)}=0.5 \cos \theta_{6}-0.866 \sin \theta_{6}, \\
& { }_{0}^{\mathrm{f}} R_{12}^{(6)}=0.5 \sin \theta_{6}+0.866 \cos \theta_{6}, \\
& { }_{0}^{\mathrm{f}} R^{(6)}{ }_{21}=-0.5 \sin \theta_{6}-0.866 \cos \theta_{6}, \\
& { }_{0}^{\mathrm{f}} R_{22}^{(6)}=0.5 \cos \theta_{6}-0.866 \sin \theta_{6} .
\end{aligned}
$$

According to Figure 1, the foot forces of the support leg $s_{k}$ can be respectively gained in the coordinate systems $\sum_{o_{k}}$ and $\sum_{f_{k}}$. The relationship is expressed as follows.

Then

$$
\left(\begin{array}{c}
F_{x}^{\left(f_{k}\right)} \\
F_{y}^{\left(f_{k}\right)} \\
F_{z}^{\left(f_{k}\right)}
\end{array}\right)={ }_{\mathrm{o}}^{\mathrm{f}} \boldsymbol{R}^{\left(s_{k}\right)}\left(\begin{array}{c}
{ }^{\mathrm{B}} F_{x}^{\left(s_{k}\right)} \\
{ }^{\mathrm{B}} F_{y}^{\left(s_{k}\right)} \\
{ }^{\mathrm{B}} F_{z}^{\left(s_{k}\right)}
\end{array}\right),
$$

When the equations from (22) to (27) are brought into Equation (28), the mathematical expressions of the foot forces of legs 1 to 6 can be respectively rewritten based on the coordinate system $\sum_{f_{k}}$.

Then

$$
\begin{aligned}
& \left\{\begin{aligned}
& F_{x}^{\left(f_{1}\right)}=\left(-0.5 \cos \theta_{1}-0.866 \sin \theta_{1}\right){ }^{\mathrm{B}} F_{x}^{\left(s_{1}\right)} \\
&+\left(-0.5 \sin \theta_{1}+0.866 \cos \theta_{1}\right)^{\mathrm{B}} F_{y}^{\left(s_{1}\right)} \\
& F_{y}^{\left(f_{1}\right)}=\left(0.5 \sin \theta_{1}-0.866 \cos \theta_{1}\right){ }^{\mathrm{B}} F_{x}^{\left(s_{1}\right)} \\
&+\left(-0.5 \cos \theta_{1}-0.866 \sin \theta_{1}\right)^{\mathrm{B}} F_{y}^{\left(s_{1}\right)} \\
& F_{z}^{\left(f_{1}\right)}={ }^{\mathrm{B}} F_{z}^{\left(s_{1}\right)}
\end{aligned}\right. \\
& \left\{\begin{array}{l}
F_{x}^{\left(f_{2}\right)}=-{ }^{\mathrm{B}} F_{x}^{\left(s_{2}\right)} \cos \theta_{2}-{ }^{\mathrm{B}} F_{y}^{\left(s_{2}\right)} \sin \theta_{2} \\
F_{y}^{\left(f_{2}\right)}={ }^{\mathrm{B}} F_{x}^{\left(s_{2}\right)} \sin \theta_{2}-{ }^{\mathrm{B}} F_{y}^{\left(s_{2}\right)} \cos \theta_{2} \\
F_{z}^{\left(f_{2}\right)}={ }^{\mathrm{B}} F_{z}^{\left(s_{2}\right)}
\end{array}\right.
\end{aligned}
$$

$$
\begin{aligned}
& \left\{\begin{aligned}
F_{x}^{\left(f_{3}\right)}= & \left(0.866 \sin \theta_{3}-0.5 \cos \theta_{3}\right)^{\mathrm{B}} F_{x}^{\left(s_{3}\right)} \\
& +\left(-0.5 \sin \theta_{3}-0.866 \cos \theta_{3}\right)^{\mathrm{B}} F_{y}^{\left(s_{3}\right)} \\
F_{y}^{\left(f_{3}\right)}= & \left(0.5 \sin \theta_{3}+0.866 \cos \theta_{3}\right)^{\mathrm{B}} F_{x}^{\left(s_{3}\right)} \\
& +\left(-0.5 \cos \theta_{3}+0.866 \sin \theta_{3}\right)^{\mathrm{B}} F_{y}^{\left(s_{3}\right)} \\
F_{z}^{\left(f_{3}\right)}= & { }^{\mathrm{B}} F_{z}^{\left(s_{3}\right)}
\end{aligned}\right. \\
& \left\{\begin{aligned}
F_{x}^{\left(f_{4}\right)}= & \left(0.5 \cos \theta_{4}+0.866 \sin \theta_{4}\right)^{\mathrm{B}} F_{x}^{\left(s_{4}\right)} \\
& +\left(0.5 \sin \theta_{4}-0.866 \cos \theta_{4}\right)^{\mathrm{B}} F_{y}^{\left(s_{4}\right)} \\
F_{y}^{\left(f_{4}\right)}= & \left(0.866 \cos \theta_{4}-0.5 \sin \theta_{4}\right)^{\mathrm{B}} F_{x}^{\left(s_{4}\right)}, \\
& +\left(0.5 \cos \theta_{4}+0.866 \sin \theta_{4}\right)^{\mathrm{B}} F_{y}^{\left(s_{4}\right)} \\
F_{z}^{\left(f_{4}\right)}= & { }^{\mathrm{B}} F_{z}^{\left(s_{4}\right)}
\end{aligned}\right. \\
& \left\{\begin{array}{l}
F_{x}^{\left(f_{5}\right)}={ }^{\mathrm{B}} F_{x}^{\left(s_{5}\right)} \cos \theta_{5}+{ }^{\mathrm{B}} F_{y}^{\left(s_{5}\right)} \sin \theta_{5} \\
F_{y}^{\left(f_{5}\right)}=-{ }^{\mathrm{B}} F_{x}^{\left(s_{5}\right)} \sin \theta_{5}+{ }^{\mathrm{B}} F_{y}^{\left(s_{5}\right)} \cos \theta_{5}, \\
F_{z}^{\left(f_{5}\right)}={ }^{\mathrm{B}} F_{z}^{\left(s_{5}\right)}
\end{array}\right. \\
& \left\{\begin{aligned}
F_{x}^{\left(f_{6}\right)}= & \left(0.5 \cos \theta_{6}-0.866 \sin \theta_{6}\right)^{\mathrm{B}} F_{x}^{\left(s_{6}\right)} \\
& +\left(0.5 \sin \theta_{6}+0.866 \cos \theta_{6}\right)^{\mathrm{B}} F_{y}^{\left(s_{6}\right)} \\
F_{y}^{\left(f_{6}\right)}= & \left(-0.5 \sin \theta_{6}-0.866 \cos \theta_{6}\right)^{\mathrm{B}} F_{x}^{\left(s_{6}\right)} . \\
& +\left(0.5 \cos \theta_{6}-0.866 \sin \theta_{6}\right)^{\mathrm{B}} F_{y}^{\left(s_{6}\right)} \\
F_{z}^{\left(f_{6}\right)}= & { }^{\mathrm{B}} F_{z}^{\left(s_{6}\right)}
\end{aligned}\right.
\end{aligned}
$$

The large-load-ratio six-legged robot can walk with the different gait. Then, the power consumption of robot mobile system under the body coordinate system can be acquired when the foot forces of the support phase among the equations from (29) to (34) are simultaneously brought into Equation (20).

\section{Simplified Power Consumption Model of Robot Mobile System under Tripod Gait}

The tripod gait is a common gait and the fastest gait for the six-legged robots. Although the tripod gait has relatively low stability relative to the quadruped gait and pentagon gait, but the tripod gait is often employed by the hexapod and six-legged robots. In this paper, the effective power consumption of robot mobile system is mainly studied under the tripod gait. When there is no special explanation, the power consumption of robot mobile system in this paper refers to the effective power consumption for the mobile system of robot. According to Equation (20), the 
mathematical model of power consumption under the tripod gait has 21 variables for the mobile system of robot. Then, it is very difficult to calculate the power consumption of robot mobile system. Hence, the experimental method and analysis means are selected to reduce the dimensionality of the power consumption model of robot mobile system. The simplified power consumption model of robot mobile system can be established under the tripod gait.

\subsection{Simplification of Force Component of Foot}

The static gait makes the six-legged robots in a statically indeterminate state. It is very difficult to calculate the foot force of each leg. The power consumption of robot mobile system is not conducive to being calculated. Then, the six-dimension force sensors are employed to collect the foot forces of legs in the three periods of gait, when the large-load-ratio six-legged robot walks on the hard flat ground. The curves are drawn based on the maximum foot force of each leg. The minimum force component of foot force is determined for each leg. The dimensionality reduction of statically indeterminate problem is realized though neglecting the minimum force components of foot forces. The negative sign in this paper only indicates that it is the opposite to the perspective positive direction.

\subsubsection{Walking Experiments of Ant-Type Tripod Gait}

The large-load-ratio six-legged robot respectively selects the ant-type tripod gait to walk along the $Y_{\mathrm{B}}$ direction of body coordinate system, when the condition of the initial angles is $\triangle \theta_{1}=\triangle \theta_{3}=\triangle \theta_{4}=\triangle \theta_{6}$, and their values are respectively set as $20^{\circ}, 30^{\circ}, 40^{\circ}, 50^{\circ}$, and $60^{\circ}$. Meanwhile, the walking parameters are respectively regarded as $0.5 \mathrm{~m}$, $0.68 \mathrm{~m}, 0.3 \mathrm{~m}, 0.3 \mathrm{~s}, 0.03 \mathrm{~m}$, and $0.1 \mathrm{~m}$ for the body height $h$, initial span $L_{\text {pi }}^{\prime}$, step pitch $s$, movement time (TM), initial lifting height $h_{\mathrm{TB}}$ of leg, and maximum swinging height $h_{\text {T-max }}$ of leg. The large-load-ratio six-legged robot does not undertake the load when the robot walks. Then, the foot forces of six legs are collected in the three gait periods. The maximum foot force of each leg is obtained. The curves of the maximum foot forces of six legs are drawn with the change in the initial angle $\triangle \theta_{i}$ under the ant-type tripod gait; they are shown in Figure 5.

Figure 5 shows that the maximum normal forces of feet gradually decrease for the legs 2 and 5 when the initial angles of abductor joints of the legs 1, 3, 4, and 6 vary from $20^{\circ}$ to $60^{\circ}$. The normal forces of feet are far greater than the tangential forces. The maximum normal forces of legs 1 to 6 are approximately the same when the initial angles $\triangle \theta_{i}$ are $60^{\circ}$. The tangential forces of legs 1 to 6 are about $0 \mathrm{~N}$ in the $y$ direction. The relation of foot force components can be obtained; it is $F_{z}^{\left(f_{k}\right)}>F_{x}^{\left(f_{k}\right)}>F_{y}^{\left(f_{k}\right)}$. Then, the foot force components in the $y$ direction can be ignored in the analysis of the power consumption of robot mobile system.

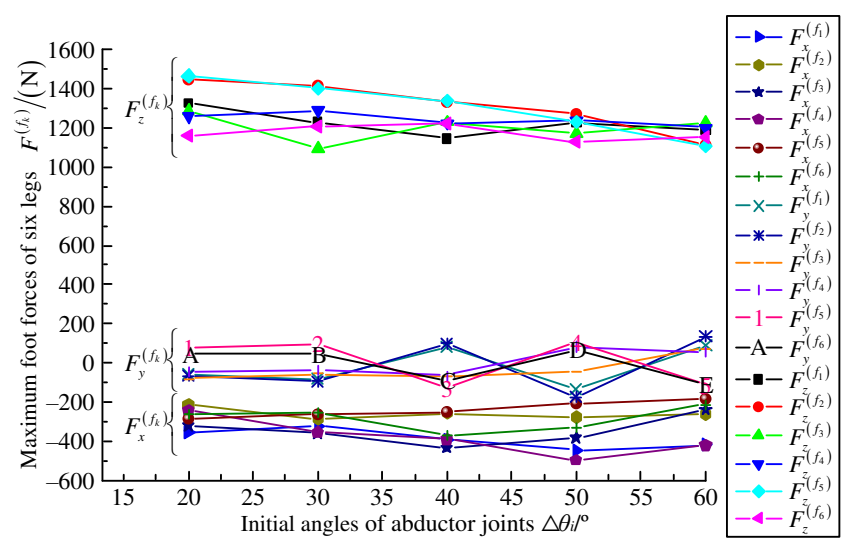

Figure 5 Curves of maximum foot forces with change in initial angle under ant-type tripod gait

\subsubsection{Walking Experiments of Crab-Type Tripod Gait}

The large-load-ratio six-legged robot respectively selects the crab-type tripod gait to walk along the $X_{\mathrm{B}}$ direction of body coordinate system, when the condition of the initial angles is $\triangle \theta_{1}=\triangle \theta_{3}=\triangle \theta_{4}=\triangle \theta_{6}$, and their values are respectively set as $0^{\circ}, 10^{\circ}, 20^{\circ}, 30^{\circ}, 40^{\circ}, 50^{\circ}$, and $60^{\circ}$. The walking parameters in the crab-type tripod gait are the same as that in the ant-type tripod gait. Then, the foot forces of six legs are collected in the three gait periods. The maximum foot force of each leg is obtained. The curves of the maximum foot forces of six legs are drawn with the change in the initial angle $\triangle \theta_{i}$ under the crab-type tripod gait; they are shown in Figure 6.

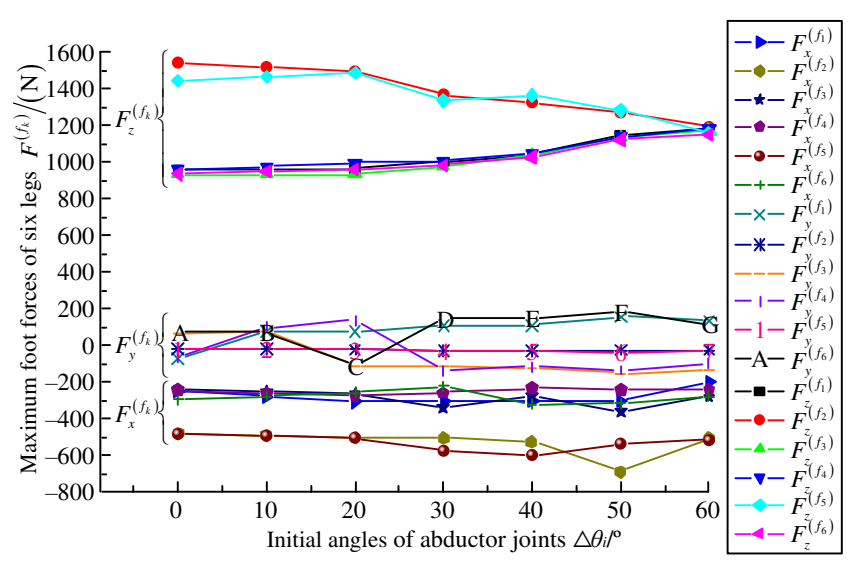

Figure 6 Curves of maximum foot forces with change in initial angle under crab-type tripod gait 
Figure 6 shows that the maximum normal forces of feet gradually decrease for the legs 2 and 5. The maximum normal forces of feet gradually increase for the legs 1, 3, 4, and 6 . The normal forces of feet are far greater than the tangential forces with the change in the abductor joints from $0^{\circ}$ to $60^{\circ}$ for the legs $1,3,4$, and 6 . The maximum normal forces of legs 1 to 6 are approximately the same when the initial angles are $60^{\circ}$. The tangential forces of legs 1 to 6 are about $0 \mathrm{~N}$ in the $y$ direction. The relation of the foot force components can be obtained; it is $F_{z}^{\left(f_{k}\right)}>$ $F_{x}^{\left(f_{k}\right)}>F_{y}^{\left(f_{k}\right)}$. Then, the foot force components in the $y$ direction can be ignored in the analysis of the power consumption of robot mobile system.

Based on Figures 5 and 6, it can be concluded that the tangential force $F_{y}^{\left(f_{k}\right)}$ can be neglected in calculating the power consumption of robot mobile system with the change in the initial angle $\triangle \theta_{i}$. When the initial angles are $60^{\circ}$, the large-load-ratio six-legged robot has the maximum carrying capacity because of approximately uniform foot force distribution for the legs 1 to 6.

\subsection{Condition Analysis of Equally Normal Foot Forces}

Based on the experimental data in section 5.1, it can be concluded that the normal foot forces of legs 1 to 6 are about the same when the initial angles of abductor joints are $60^{\circ}$. To perform the equally normal foot forces is beneficial to develop the furthest capabilities of support legs and make the large-load-ratio six-legged robot carry more materials. In view of all the nice features in the aspects of stability, walking speed, foot force distribution, and so on, the mixed-type II tripod gait is preferred to be employed for the large-load-ratio six-legged robot. Then, the conditions of equally normal foot forces are analyzed under the mixed-type II tripod gait.

The large-load-ratio six-legged robot walks from the initial poses under the mixed-type II tripod gait. Meanwhile, the initial attitudes of abductor joints, hip joints, and knee joints are $\triangle \theta_{1}=\triangle \theta_{3}=\triangle \theta_{4}=\triangle \theta_{6}=60^{\circ}, \triangle$ $\theta_{2}=\triangle \theta_{5}=0^{\circ}, \quad \beta^{\prime}{ }_{1}=\beta^{\prime}{ }_{2}=\beta^{\prime}{ }_{3}=\beta^{\prime}{ }_{4}=\beta^{\prime}{ }_{5}=\beta^{\prime}{ }_{6}$, and $\beta_{1}=\beta_{2}=\beta_{3}=\beta_{4}=$ $\beta_{5}=\beta_{6}$, respectively. The legs 2,4 , and 6 firstly stride forward a distance of $s / 2$, and they will transform from the transfer phase to the support phase. Then, the legs 1,3, and 5 mobile a distance of $s$ with the body of robot, and they will transform from the support phase to the transfer phase. The initial spans of the legs 2, 4, and 6 are equal; they are $L_{\mathrm{P} 2}^{\prime}=L_{\mathrm{P} 4}^{\prime}=L_{\mathrm{P} 6}^{\prime}$.

The scheme of the support phase of large-load-ratio six-legged robot under the mixture-type II tripod gait is shown in Figure 7.

In Figure $7, r_{\mathrm{bp}}$ is the effective radius of the bearing platform. $s_{\mathrm{u}}$ and $s_{\mathrm{d}}$ are respectively defined as the up point of foot and down point of foot when the robot walks. The components of position vector for the foot end of support leg $s_{k}$ are respectively regarded as ${ }^{\mathrm{B}} P_{\mathrm{F} x}^{\left(s_{k}\right)},{ }^{\mathrm{B}} P_{\mathrm{Fy}}^{\left(s_{k}\right)}$, and ${ }^{\mathrm{B}} P_{\mathrm{F} z}^{\left(s_{k}\right)}$ in the body coordinate system $\sum_{\mathrm{B}}$. The robot is assumed to walk at a constant speed and in straight lines. The height remains unchanged between the body and the ground. The projection trajectory of the foot end of each leg in the $y$ direction is parallel to the moving track of the gravity center of body. Based on Figure 2, the expression of principal moment of the robot system can be expressed as follows. Then

$$
\boldsymbol{J}_{\mathrm{M}}{ }^{\mathrm{B}} \boldsymbol{F}_{\mathrm{s}}=\boldsymbol{M}_{\mathrm{B}},
$$

where $\boldsymbol{J}_{\mathrm{M}}$ is the position matrix of foot ends of the support phase in the body coordinate system $\Sigma_{\mathrm{B}}$. Meanwhile, the correlation matrix can be represented as follows.

$$
\begin{aligned}
& \boldsymbol{J}_{\mathrm{M}}=\left(\begin{array}{lllll}
{ }^{\mathrm{B}} \boldsymbol{P}_{\mathrm{F}}^{\left(s_{\mathrm{l}}\right)} & \ldots & { }^{\mathrm{B}} \boldsymbol{P}_{\mathrm{F}}^{\left(s_{k}\right)} & \ldots & { }^{\mathrm{B}} \boldsymbol{P}_{\mathrm{F}}^{\left(s_{u}\right)}
\end{array}\right) \in \mathbf{R}^{3 \times 3 u}, \\
& { }^{\mathrm{B}} \boldsymbol{P}_{\mathrm{F}}^{\left(s_{k}\right)}=\left(\begin{array}{ccc}
0 & -{ }^{\mathrm{B}} P_{\mathrm{F} z}^{\left(s_{k}\right)} & { }^{\mathrm{B}} P_{\mathrm{F} y}^{\left(s_{k}\right)} \\
{ }^{\mathrm{B}} P_{\mathrm{F} z}^{\left(s_{k}\right)} & 0 & -{ }^{\mathrm{B}} P_{\mathrm{F} x}^{\left(s_{k}\right)} \\
-{ }^{\mathrm{B}} P_{\mathrm{Fy}}^{\left(s_{k}\right)} & { }^{\mathrm{B}} P_{\mathrm{F} x}^{\left(s_{k}\right)} & 0
\end{array}\right) \in \mathbf{R}^{3 \times 3} .
\end{aligned}
$$

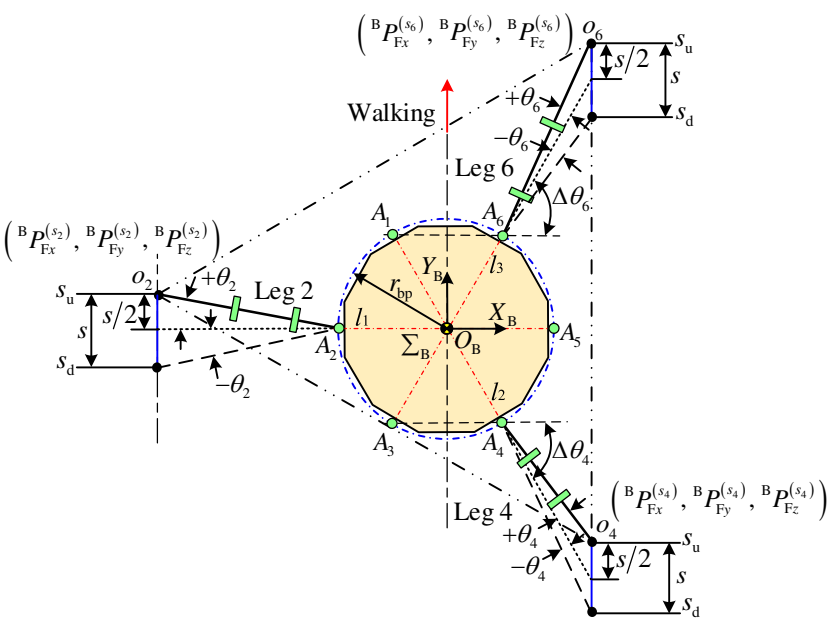

Figure 7 Scheme of support phase under mixture-type II tripod gait [27]

In general, the large-load-ratio six-legged robot is assumed to be unaffected by other external principal vectors and principal moments except for the mass $m_{\mathrm{L}}$ of robot and mass $m_{\mathrm{R}}$ of carrying materials. The tangential forces are $0 \mathrm{~N}$ for the support legs. The principal vector $\boldsymbol{F}_{\mathrm{B}}$ and principal moment $\boldsymbol{M}_{\mathrm{B}}$ of the robot system can be written as follows. Then 


$$
\left(\begin{array}{c}
\boldsymbol{F}_{\mathrm{B}} \\
\boldsymbol{M}_{\mathrm{B}}
\end{array}\right)=\left(\begin{array}{llllll}
0 & 0 & m_{\mathrm{L}} g+m_{\mathrm{R}} g & 0 & 0 & 0
\end{array}\right)^{\mathrm{T}} \in \mathbf{R}^{6 \times 1},
$$

The legs 2, 4, and 6 are assumed to be located in the support phase under the tripod gait. Meanwhile, the legs 1, 3 , and 5 lie in the transfer phase. The normal forces of support legs depend on the mass $m_{\mathrm{L}}$ of robot and mass $m_{\mathrm{R}}$ of carrying materials. Then

$$
\left(\begin{array}{c}
{ }^{\mathrm{B}} \boldsymbol{F}_{s_{2}} \\
{ }^{\mathrm{B}} \boldsymbol{F}_{s_{4}} \\
{ }^{\mathrm{B}} \boldsymbol{F}_{s_{6}}
\end{array}\right)=\left(\begin{array}{llllllllll}
0 & 0 & { }^{\mathrm{B}} F_{z}^{\left(s_{2}\right)} & 0 & 0 & { }^{\mathrm{B}} F_{z}^{\left(s_{4}\right)} & 0 & 0 & { }^{\mathrm{B}} F_{z}^{\left(s_{6}\right)}
\end{array}\right)^{\mathrm{T}},
$$

The generalized force matrix of the support phase can be written for the large-load-ratio six-legged robot under the tripod gait. Then

$$
\left(\begin{array}{c}
\boldsymbol{F}_{\mathrm{B}} \\
\boldsymbol{M}_{\mathrm{B}}
\end{array}\right)=\left(\begin{array}{ccc}
\boldsymbol{I}_{3 \times 3} & \boldsymbol{I}_{3 \times 3} & \boldsymbol{I}_{3 \times 3} \\
{ }^{\mathrm{B}} \boldsymbol{P}_{\mathrm{F}}^{\left(s_{2}\right)} & { }^{\mathrm{B}} \boldsymbol{P}_{\mathrm{F}}^{\left(s_{4}\right)} & { }^{\mathrm{B}} \boldsymbol{P}_{\mathrm{F}}^{\left(s_{6}\right)}
\end{array}\right)\left(\begin{array}{c}
{ }^{\mathrm{B}} \boldsymbol{F}_{s_{2}} \\
{ }^{\mathrm{B}} \boldsymbol{F}_{s_{4}} \\
{ }^{\mathrm{B}} \boldsymbol{F}_{s_{6}}
\end{array}\right),
$$

where $\boldsymbol{I}_{3 \times 3}$ is the $3 \times 3$ identity matrix.

Equations (35), (36), and (37) are brought into Equation (38). Then, the mathematical expressions can be obtained as follows.

$$
\left\{\begin{array}{l}
{ }^{\mathrm{B}} F_{z}^{\left(s_{2}\right)}+{ }^{\mathrm{B}} F_{z}^{\left(s_{4}\right)}+{ }^{\mathrm{B}} F_{z}^{\left(s_{6}\right)}=m_{\mathrm{L}} g+m_{\mathrm{R}} g \\
{ }^{\mathrm{B}} P_{\mathrm{F} y}^{\left(s_{2}\right)} \times{ }^{\mathrm{B}} F_{z}^{\left(s_{2}\right)}+{ }^{\mathrm{B}} P_{\mathrm{F} y}^{\left(s_{4}\right)} \times{ }^{\mathrm{B}} F_{z}^{\left(s_{4}\right)}+{ }^{\mathrm{B}} P_{\mathrm{Fy}}^{\left(s_{6}\right)} \times{ }^{\mathrm{B}} F_{z}^{\left(s_{6}\right)}=0, \\
{ }^{\mathrm{B}} P_{\mathrm{F} x}^{\left(s_{2}\right)} \times{ }^{\mathrm{B}} F_{z}^{\left(s_{2}\right)}+{ }^{\mathrm{B}} P_{\mathrm{F} x}^{\left(s_{4}\right)} \times{ }^{\mathrm{B}} F_{z}^{\left(s_{4}\right)}+{ }^{\mathrm{B}} P_{\mathrm{F} x}^{\left(s_{6}\right)} \times{ }^{\mathrm{B}} F_{z}^{\left(s_{6}\right)}=0
\end{array}\right.
$$

According to Figure 7, the foot forces of support legs in the $x$ direction can be written in the body coordinate system $\sum_{\mathrm{B}}$. Then

$$
\left(\begin{array}{c}
{ }^{\mathrm{B}} P_{\mathrm{F} x}^{\left(s_{2}\right)} \\
{ }^{\mathrm{B}} P_{\mathrm{F} x}^{\left(s_{4}\right)} \\
{ }^{\mathrm{B}} P_{\mathrm{F} x}^{\left(s_{6}\right)}
\end{array}\right)=\left(\begin{array}{c}
-\left(r_{\mathrm{bp}}+L_{\mathrm{P} 2}^{\prime}\right) \\
\left(r_{\mathrm{bp}}+L_{\mathrm{P} 4}^{\prime}\right) \cos 60^{\circ} \\
\left(r_{\mathrm{bp}}+L_{\mathrm{P} 6}^{\prime}\right) \cos 60^{\circ}
\end{array}\right),
$$

Equation (40) can be brought into Equation (39) based on the initial attitudes of abductor joints and the initial spans of leg 2, 4, and 6: $\triangle \theta_{1}=\triangle \theta_{3}=\triangle \theta_{4}=\triangle \theta_{6}=60^{\circ}$, $\theta_{2}=\triangle \theta_{5}=0^{\circ}$, and $L_{\mathrm{P} 2}^{\prime}=L_{\mathrm{P} 4}^{\prime}=L_{\mathrm{P} 6}^{\prime}$. Then

$$
\left(\begin{array}{c}
{ }^{\mathrm{B}} F_{z}^{\left(s_{2}\right)} \\
{ }^{\mathrm{B}} F_{z}^{\left(s_{4}\right)}+{ }^{\mathrm{B}} F_{z}^{\left(s_{6}\right)}
\end{array}\right)=\left(\begin{array}{l}
\frac{1}{3}\left(m_{\mathrm{L}} g+m_{\mathrm{R}} g\right) \\
\frac{2}{3}\left(m_{\mathrm{L}} g+m_{\mathrm{R}} g\right)
\end{array}\right),
$$

The feet of the support phase move back relative distance $s$ when the body of large-load-ratio six-legged robot moves forward a step pitch $s$. To make it easier to facilitate the condition analysis of equally normal foot forces, the robot body is assumed to be stationary. Hence, all support points of the support phase move from the up point $s_{\mathrm{u}}$ to the down point $s_{\mathrm{d}}$. The $y$ coordinates of foot ends in the body coordinate system $\Sigma_{\mathrm{B}}$ can be obtained for the legs 2, 4, and 6. Then

$$
\left(\begin{array}{c}
{ }^{\mathrm{B}} P_{\mathrm{F} y}^{\left(s_{2}\right)} \\
{ }^{\mathrm{B}} P_{\mathrm{F} y}^{\left(s_{4}\right)} \\
{ }^{\mathrm{B}} P_{\mathrm{F} y}^{\left(s_{6}\right)}
\end{array}\right)=\left(\begin{array}{c}
-s / 2 \\
-\left(r_{\mathrm{bp}}+L_{\mathrm{P} 4}^{\prime}\right) \sin 60^{\circ}-s / 2 \\
\left(r_{\mathrm{bp}}+L_{\mathrm{P} 6}^{\prime}\right) \sin 60^{\circ}-s / 2
\end{array}\right),
$$

To conveniently facilitate the following research, the initial spans of leg 2, 4, and 6 are $L_{\mathrm{P} 2}^{\prime}=L_{\mathrm{P} 4}^{\prime}=L_{\mathrm{P} 6}^{\prime}=L_{\mathrm{PS}}^{\prime}$. Equations (41) and (42) are introduced into Equation (39). The mathematical expression can be gained. Then

$$
\begin{aligned}
& { }^{\mathrm{B}} F_{z}^{\left(s_{4}\right)} \times\left(\frac{\sqrt{3}}{2}\left(r_{\mathrm{bp}}+L_{\mathrm{PS}}^{\prime}\right)+\frac{s}{2}\right)+\frac{1}{6}\left(m_{\mathrm{L}} g+m_{\mathrm{R}} g\right) s \\
& ={ }^{\mathrm{B}} F_{z}^{\left(s_{6}\right)} \times\left(\frac{\sqrt{3}}{2}\left(r_{\mathrm{bp}}+L_{\mathrm{PS}}^{\prime}\right)-\frac{s}{2}\right)
\end{aligned}
$$

According to the initial attitudes of abductor joints: $\triangle$ $\theta_{1}=\triangle \theta_{3}=\triangle \theta_{4}=\triangle \theta_{6}=60^{\circ}$ and $\triangle \theta_{2}=\triangle \theta_{5}=0^{\circ}$, it can be concluded that a regular triangle is constructed by the support feet. The regular triangle can provide the maximum stability margin $d_{\mathrm{M} \text {-max }}$ for the large-load- ratio six-legged robot. Then

$$
d_{\mathrm{M}-\max }=\frac{1}{2}\left(r_{\mathrm{bp}}+L_{\mathrm{PS}}^{\prime}\right),
$$

Actually, the maximum stability margin $d_{\mathrm{M}-\max }$ limits the range of step pitch $s$ based on the stability of robot. Then

$$
s \cos 30^{\circ} \leq \frac{1}{2}\left(r_{\mathrm{bp}}+L_{\mathrm{PS}}^{\prime}\right),
$$

Based on Equation (45), the maximum step pitch $s_{\max }$ can be written. Then 


$$
S_{\max }=\frac{\sqrt{3}}{3}\left(r_{\mathrm{bp}}+L_{\mathrm{PS}}^{\prime}\right)
$$

The maximum step pitch $s_{\max }$ and Equation (41) are brought into Equation (43). The normal foot forces of legs 4 and 6 can be obtained. Then

$$
\left(\begin{array}{c}
{ }^{\mathrm{B}} F_{z}^{\left(s_{4}\right)} \\
{ }^{\mathrm{B}} F_{z}^{\left(s_{6}\right)}
\end{array}\right)=\left(\begin{array}{l}
\frac{1}{6}\left(m_{\mathrm{L}} g+m_{\mathrm{R}} g\right) \\
\frac{3}{6}\left(m_{\mathrm{L}} g+m_{\mathrm{R}} g\right)
\end{array}\right),
$$

According to Equation (47), it can be concluded that the normal foot force of leg 6 is greater than that of legs 2 and 4 when the robot is at the critical point between stability and instability. It is not good for the large-load- ratio six-legged robot to carry heavier materials.

The step pitch $s=s_{\max } / 3$ and Equation (41) are introduced into Equation (43). The normal foot forces of legs 4 and 6 can be regained. Then

$$
\left(\begin{array}{c}
{ }^{\mathrm{B}} F_{z}^{\left(s_{4}\right)} \\
{ }^{\mathrm{B}} F_{z}^{\left(s_{6}\right)}
\end{array}\right)=\left(\begin{array}{c}
\frac{5}{18}\left(m_{\mathrm{L}} g+m_{\mathrm{R}} g\right) \\
\frac{7}{18}\left(m_{\mathrm{L}} g+m_{\mathrm{R}} g\right)
\end{array}\right),
$$

Based on Figure 7, the step pitch $s=s_{\max } / 3$, and effective radius $r_{\mathrm{bp}}$ of bearing platform, the ranges of rotational angles can be calculated for the abductor joints of legs 2, 4, and 6 when the length of initial span $L_{\mathrm{PS}}^{\prime}$ is $0.68 \mathrm{~m}$. Then

$$
\left\{\begin{array}{l}
-8.7^{\circ} \leq \theta_{2} \leq 8.7^{\circ} \\
-3.8^{\circ} \leq \theta_{4} \leq 5^{\circ} \\
-5^{\circ} \leq \theta_{6} \leq 3.8^{\circ}
\end{array} .\right.
$$

According to Equation (49), it is concluded that the rotational angles of abductor joints have homologous characteristics. Namely, they can synchronously take the positive value, the negative value, or the maximum value.

Based on Equation (48) and the technology indexes of robot, it can be think that the normal foot forces of legs 2 , 4 , and 6 are approximately equal and their value are $\left(m_{\mathrm{L}} g+m_{\mathrm{R}} g\right) / 3$, when the value of step pitch $s$ is $s_{\max } / 3$ and the robot walks in accordance with Figure 7 . Then, the errors of normal foot forces are less than $15 \%$ between the actual value and the approximate value. They are within the allowable error range. Hence, Equation (49) is the equal conditions of normal foot forces under the tripod gait for the large-load-ratio six-legged robot.
Due to the periodic alternate between the support phase and the transfer phase, the above analysis and regulations are equally applicable when the legs 1, 3 and 5 are in the support phase, and the legs 2, 4 and 6 are in the transfer phase. According to the symmetry of six legs evenly distributed body, it is only necessary to change leg 2 into leg 5 , leg 4 into leg 3 , and leg 6 into leg 1 . The specific analysis is no longer in detail here.

\subsection{Simplified Model of Power Consumption of Robot Mobile System}

Based on the above analysis, the tangential force $F_{y}^{\left(f_{k}\right)}$ of support leg can be neglected under the tripod gait. The normal foot forces of support legs are about $\left(m_{\mathrm{L}} \mathrm{g}+m_{\mathrm{R}} \mathrm{g}\right) / 3$ when the value of step pitch $s$ is $s_{\max } / 3$, and the robot walks under the mixed-type II tripod gait. Hence, the simplified power consumption model of robot mobile system can be written when the robot walks from the initial attitudes under the mixed-type II or ant-type tripod gait, the initial attitudes of abductor joints are $\triangle \theta_{1}=\triangle \theta_{3}=\triangle \theta_{4}=\triangle \theta_{6}=60^{\circ}$ and $\triangle \theta_{2}=\triangle \theta_{5}=0^{\circ}$, and the legs 2,4 , and 6 are in the support phase. Then

$$
\begin{aligned}
P_{\mathrm{sum}}=\sum_{k=2,4,6} & \left(\mid 0.56 F_{x}^{\left(f_{k}\right)}\left(l_{\mathrm{t}} \sin \beta_{k}^{\prime}+l_{\mathrm{s}} \sin \beta_{k}\right)\right. \\
& +\left(0.187\left(m_{\mathrm{L}} g+m_{\mathrm{R}} g\right)-0.28 m_{\mathrm{s}} g\right) \\
& \times\left(l_{\mathrm{t}} \cos \beta_{k}^{\prime}+l_{\mathrm{s}} \cos \beta_{k}\right)-0.28\left(m_{\mathrm{t}} g+m_{\mathrm{s}} g\right) \\
& \times l_{\mathrm{t}} \cos \beta_{k}^{\prime}|+| 0.56 F_{x}^{\left(f_{k}\right)} l_{\mathrm{s}} \sin \beta_{k} \\
& \left.+\left(0.187\left(m_{\mathrm{L}} g+m_{\mathrm{R}} g\right)-0.28 m_{\mathrm{s}} g\right) l_{\mathrm{s}} \cos \beta_{k} \mid\right) \\
+ & \sum_{e=1,3,5}\left(\mid 0.28 m_{\mathrm{t}} g l_{\mathrm{t}} \cos \beta_{e}^{\prime}+0.56 m_{\mathrm{s}} g l_{\mathrm{t}} \cos \beta_{e}^{\prime}\right. \\
& \left.+0.28 m_{\mathrm{s}} g l_{\mathrm{s}} \cos \beta_{e}|+| 0.28 m_{\mathrm{s}} g l_{\mathrm{s}} \cos \beta_{e} \mid\right)
\end{aligned}
$$

The tangential force $F_{y}^{\left(f_{k}\right)}=0$ is brought into Equations (30), (32), and (34). The mathematical expressions the foot forces can be obtained for the legs 2, 4, and 6 . Then

$$
\left(\begin{array}{l}
F_{x}^{\left(f_{2}\right)} \\
F_{x}^{\left(f_{4}\right)} \\
F_{x}^{\left(f_{6}\right)}
\end{array}\right)=\left(\begin{array}{c}
-{ }^{\mathrm{B}} F_{x}^{\left(s_{2}\right)} / \cos \theta_{2} \\
{ }^{\mathrm{B}} F_{x}^{\left(s_{4}\right)} / \sin \left(30^{\circ}+\theta_{4}\right) \\
{ }^{\mathrm{B}} F_{x}^{\left(s_{6}\right)} / \sin \left(30^{\circ}-\theta_{6}\right)
\end{array}\right),
$$

Equation (51) can be introduced into Equation (50). The expanded form of simplified mathematical model for the power consumption of robot mobile system can be written when the large-load-ratio six-legged robot walks under the mixed-type II tripod gait. Then 


$$
P_{\text {sum }}=P_{\text {sum } 2}+P_{\text {sum } 4}+P_{\text {sum6 } 6}+P_{\text {sum } 1}+P_{\text {sum3 }}+P_{\text {sum5 }},
$$

where $P_{\text {sum } 2}, P_{\text {sum } 4}$, and $P_{\text {sum6 }}$ are the power consumption of legs 2,4 , and 6 in the support phase. $P_{\text {sum } 1}, P_{\text {sum } 3}$, and $P_{\text {sum5 }}$ represent the power consumption of legs 1,3 , and 5 in the transfer phase. Meanwhile, their mathematical expressions can be written as follows.

$$
\begin{aligned}
& P_{\text {sum } 1}=\mid 0.28 m_{\mathrm{t}} g l_{\mathrm{t}} \cos \beta_{1}^{\prime}+0.56 m_{\mathrm{s}} g l_{\mathrm{t}} \cos \beta_{1}^{\prime} \\
& +\left.0.28 m_{\mathrm{s}} g l_{\mathrm{s}} \cos \beta_{1}|+| 0.28 m_{\mathrm{s}} g l_{\mathrm{s}} \cos \beta_{1}\right|^{\prime} \\
& P_{\text {sum } 2}=-0.56{ }^{\mathrm{B}} F_{x}^{\left(s_{2}\right)}\left(l_{\mathrm{t}} \sin \beta_{2}^{\prime}+l_{\mathrm{s}} \sin \beta_{2}\right) / \cos \theta_{2} \\
& +\left(0.187\left(m_{\mathrm{L}} g+m_{\mathrm{R}} g\right)-0.28 m_{\mathrm{s}} g\right) \\
& \times\left(l_{\mathrm{t}} \cos \beta_{2}^{\prime}+l_{\mathrm{s}} \cos \beta_{2}\right)-0.28\left(m_{\mathrm{t}} g+m_{\mathrm{s}} g\right), \\
& \times l_{\mathrm{t}} \cos \beta_{2}^{\prime}|+|-0.56^{\mathrm{B}} F_{x}^{\left(s_{2}\right)} l_{\mathrm{s}} \sin \beta_{2} / \cos \theta_{2} \\
& +\left(0.187\left(m_{\mathrm{L}} g+m_{\mathrm{R}} g\right)-0.28 m_{\mathrm{s}} g\right) l_{\mathrm{s}} \cos \beta_{2} \\
& P_{\text {sum3 }}=\mid 0.28 m_{\mathrm{t}} g l_{\mathrm{t}} \cos \beta_{3}^{\prime}+0.56 m_{\mathrm{s}} g l_{\mathrm{t}} \cos \beta_{3}^{\prime} \\
& +0.28 m_{\mathrm{s}} g l_{\mathrm{s}} \cos \beta_{3}|+| 0.28 m_{\mathrm{s}} g l_{\mathrm{s}} \cos \beta_{3} \mid{ }^{\prime} \\
& P_{\text {sum } 4}=\mid 0.56^{\mathrm{B}} F_{x}^{\left(s_{4}\right)}\left(l_{\mathrm{t}} \sin \beta_{4}^{\prime}+l_{\mathrm{s}} \sin \beta_{4}\right) / \sin \left(30^{\circ}+\theta_{4}\right) \\
& +\left(0.187\left(m_{\mathrm{L}} g+m_{\mathrm{R}} g\right)-0.28 m_{\mathrm{s}} g\right) \\
& \times\left(l_{\mathrm{t}} \cos \beta_{4}^{\prime}+l_{\mathrm{s}} \cos \beta_{4}\right)-0.28\left(m_{\mathrm{t}} g+m_{\mathrm{s}} g\right) \quad, \\
& \times l_{\mathrm{t}} \cos \beta_{4}^{\prime}|+| 0.56^{\mathrm{B}} F_{x}^{\left(s_{4}\right)} l_{\mathrm{s}} \sin \beta_{4} / \sin \left(30^{\circ}+\theta_{4}\right) \\
& +\left(0.187\left(m_{\mathrm{L}} g+m_{\mathrm{R}} g\right)-0.28 m_{\mathrm{s}} g\right) l_{\mathrm{s}} \cos \beta_{4} \mid \\
& P_{\text {sum5 }}=\mid 0.28 m_{\mathrm{t}} g l_{\mathrm{t}} \cos \beta_{5}^{\prime}+0.56 m_{\mathrm{s}} g l_{\mathrm{t}} \cos \beta_{5}^{\prime} \\
& +\left.0.28 m_{\mathrm{s}} g l_{\mathrm{s}} \cos \beta_{5}|+| 0.28 m_{\mathrm{s}} g l_{\mathrm{s}} \cos \beta_{5}\right|^{\prime} \\
& P_{\text {sum } 6}=\mid 0.56^{\mathrm{B}} F_{x}^{\left(s_{6}\right)}\left(l_{\mathrm{t}} \sin \beta_{6}^{\prime}+l_{\mathrm{s}} \sin \beta_{6}\right) / \sin \left(30^{\circ}-\theta_{6}\right) \\
& +\left(0.187\left(m_{\mathrm{L}} g+m_{\mathrm{R}} g\right)-0.28 m_{\mathrm{s}} g\right) \\
& \times\left(l_{\mathrm{t}} \cos \beta_{6}^{\prime}+l_{\mathrm{s}} \cos \beta_{6}\right)-0.28\left(m_{\mathrm{t}} g+m_{\mathrm{s}} g\right) \\
& \times l_{\mathrm{t}} \cos \beta_{6}^{\prime}|+| 0.56^{\mathrm{B}} F_{x}^{\left(s_{6}\right)} l_{\mathrm{s}} \sin \beta_{6} / \sin \left(30^{\circ}-\theta_{6}\right) \\
& +\left(0.187\left(m_{\mathrm{L}} g+m_{\mathrm{R}} g\right)-0.28 m_{\mathrm{s}} g\right) l_{\mathrm{s}} \cos \beta_{6} \mid
\end{aligned}
$$

\section{Constraint Condition of Robot Stable Walking under Tripod Gait}

To realize the stable walking of robot, the certain inequality and equality constraints need to be satisfied for the large-load-ratio six-legged robot under the tripod gait.

\subsection{Inequality Constraint Conditions}

\subsubsection{Constraint Condition of Normal Foot Force}

To maintain the contact between the support leg and the ground, the normal foot force is nonnegative. The inequality constraint condition of normal foot force can be written for the support leg $s_{k}$. Then

$$
{ }^{\mathrm{B}} F_{z}^{\left(s_{k}\right)} \geq 0 .
$$

\subsubsection{Constraint Condition of Friction between Foot and Ground}

To ensure the stable walking of robot, it is assumed that there is no slip between the feet and the ground. The large-load-ratio six-legged robot has the universal walking ability. To highlight the relationship between the ground friction and the components of foot force in the $x$ direction and $y$ direction, the frictional constraint condition can be written for the support leg $s_{k}$. Then

$$
\sqrt{\left({ }^{\mathrm{B}} F_{x}^{\left(s_{k}\right)}\right)^{2}+\left({ }^{\mathrm{B}} F_{y}^{\left(s_{k}\right)}\right)^{2}} \leq \mu^{\mathrm{B}} F_{z}^{\left(s_{k}\right)},
$$

where $\mu$ is the coefficient of ground static friction.

The tangential force $F_{y}^{\left(f_{k}\right)}=0$ is introduced into Equations (48), (50), and (52). The mathematical expressions of tangential forces in the body coordinate system $\sum_{\mathrm{B}}$ can be obtained for the legs 2,4 , and 6 . Then

$$
\left\{\begin{array}{l}
{ }^{\mathrm{B}} F_{y}^{\left(s_{2}\right)}={ }^{\mathrm{B}} F_{x}^{\left(s_{2}\right)} \tan \theta_{2} \\
{ }^{\mathrm{B}} F_{y}^{\left(s_{4}\right)}=-{ }^{\mathrm{B}} F_{x}^{\left(s_{4}\right)} \cot \left(30^{\circ}+\theta_{4}\right), \\
{ }^{\mathrm{B}} F_{y}^{\left(s_{6}\right)}={ }^{\mathrm{B}} F_{x}^{\left(s_{6}\right)} \cot \left(30^{\circ}-\theta_{6}\right)
\end{array}\right.
$$

When the normal foot forces of support legs and Equation (55) are brought into Equation (54), the constraint conditions of foot forces in the $x$ direction can be written for the legs 2,4 , and 6 . Then

$$
\left\{\begin{array}{rl}
-\frac{1}{3}\left(m_{\mathrm{L}} g\right. & \left.+m_{\mathrm{R}} g\right) \mu \cos \theta_{2} \leq{ }^{\mathrm{B}} F_{x}^{\left(s_{2}\right)} \\
& \leq \frac{1}{3}\left(m_{\mathrm{L}} g+m_{\mathrm{R}} g\right) \mu \cos \theta_{2} \\
-\frac{1}{3}\left(m_{\mathrm{L}} g\right. & \left.+m_{\mathrm{R}} g\right) \mu \sin \left(30^{\circ}+\theta_{4}\right) \leq{ }^{\mathrm{B}} F_{x}^{\left(s_{4}\right)} \\
\leq & \frac{1}{3}\left(m_{\mathrm{L}} g+m_{\mathrm{R}} g\right) \mu \sin \left(30^{\circ}+\theta_{4}\right) \\
-\frac{1}{3}\left(m_{\mathrm{L}} g\right. & \left.+m_{\mathrm{R}} g\right) \mu \sin \left(30^{\circ}-\theta_{6}\right) \leq{ }^{\mathrm{B}} F_{x}^{\left(s_{6}\right)} \\
\leq & \frac{1}{3}\left(m_{\mathrm{L}} g+m_{\mathrm{R}} g\right) \mu \sin \left(30^{\circ}-\theta_{6}\right)
\end{array} .\right.
$$

\subsubsection{Constraint Condition of Articulated Driving}

Due to the burden of carrying large quality materials for 
the large-load-ratio six-legged robot, it is necessary to consider the restrictions of the driving abilities of articulated servo motors. The driving torque of each joint should be within its maximum allowable range. The maximum articulated torque should be determined by comparing the articulated load torque calculated by the maximum allowable current of driver with the articulated load torque calculated by the locked-rotor torque of servo motor. The weight of the robot and materials is entirely borne by the support phase. In the aspect of homogeneous joints, the torques of joints in the support phase are greater than that in the transfer phase. Then, the articulated torques need to be restricted in the support phase.

The articulated torques in the support phase can be determined by both the foot forces and the motion of robot. The constraint condition of articulated driving is written for the total torque $M_{\mathrm{A}}^{\left(s_{k}\right)}$ of abductor joint, the total torque $M_{\mathrm{H}}^{\left(s_{k}\right)}$ of hip joint, and the total torque $M_{\mathrm{K}}^{\left(s_{k}\right)}$ of knee joint under the support leg $s_{k}$.

Then

$$
-\left(\begin{array}{l}
M_{\mathrm{A} \_ \text {max }}^{\left(s_{k}\right)} \\
M_{\mathrm{H} \_ \text {max }}^{\left(s_{k}\right)} \\
M_{\mathrm{K} \_ \text {max }}^{\left(s_{k}\right)}
\end{array}\right) \leq\left(\begin{array}{l}
M_{\mathrm{A}}^{\left(s_{k}\right)} \\
M_{\mathrm{H}}^{\left(s_{k}\right)} \\
M_{\mathrm{K}}^{\left(s_{k}\right)}
\end{array}\right) \leq\left(\begin{array}{l}
M_{\mathrm{A} \_\max }^{\left(s_{k}\right)} \\
M_{\mathrm{H} \_\max }^{\left(s_{k}\right)} \\
M_{\mathrm{K} \_\max }^{\left(s_{k}\right)}
\end{array}\right),
$$

where $M_{j_{-} \text {max }}^{\left(s_{k}\right)}(j=\mathrm{A}, \mathrm{H}, \mathrm{K})$ is the maximum allowable torque of joint $j$ in the support leg $s_{k}$.

The models of drivers at the 18 driving joints are Elmo SOL-WHI 15/60 E02 whose the maximum allowable current is $15 \mathrm{~A}$. Based on the performance parameters of driving devices and actuating devices in Table 1, the maximum allowable torques of joints can be obtained for the abductor joint, hip joint, and knee joint of the support leg $s_{k}$.

Then

$$
\left\{\begin{array}{l}
M_{\mathrm{A} \_ \text {max }}^{\left(s_{k}\right)}=K_{\mathrm{MA}}^{\left(s_{k}\right)} i_{\mathrm{CA} \_ \text {max }}^{\left(s_{k}\right)} i_{\mathrm{A}}^{\left(s_{k}\right)} \eta_{\mathrm{A}}^{\left(s_{k}\right)}=620 \mathrm{~N} \cdot \mathrm{m} \\
M_{\mathrm{H} \_ \text {max }}^{\left(s_{k}\right)}=K_{\mathrm{MH}}^{\left(s_{k}\right)} i_{\mathrm{CH} \_ \text {max }}^{\left(s_{k}\right)} i_{\mathrm{H}}^{\left(s_{k}\right)} \eta_{\mathrm{H}}^{\left(s_{k}\right)}=1286 \mathrm{~N} \cdot \mathrm{m}, \\
M_{\mathrm{K} \_ \text {max }}^{\left(s_{k}\right)}=K_{\mathrm{MK}}^{\left(s_{k}\right)} i_{\mathrm{CK} \_ \text {max }}^{\left(s_{k}\right)} i_{\mathrm{K}}^{\left(s_{k}\right)} \eta_{\mathrm{K}}^{\left(s_{k}\right)}=1286 \mathrm{~N} \cdot \mathrm{m}
\end{array}\right.
$$

By comparison, it can be concluded that the maximum articulated torque calculated by the maximum allowable current of driver is far less than that of calculating through the locked-rotor torque of servo motor. Therefore, the articulated torques of robot can be limited by Equation (58). Based on Equations (12) and (58), the constraints of articulated torques are converted to the constraints of the foot forces and rotational angles of joints.

Then

$$
\left\{\begin{aligned}
-620 \mathrm{~N} \cdot \mathrm{m} & \leq F_{y}^{\left(f_{k}\right)}\left(l_{\mathrm{c}}+l_{\mathrm{t}} \cos \beta_{k}^{\prime}+l_{\mathrm{s}} \cos \beta_{k}\right) \\
& \leq 620 \mathrm{~N} \cdot \mathrm{m} \\
-1286 \mathrm{~N} \cdot \mathrm{m} & \leq F_{x}^{\left(f_{k}\right)}\left(l_{\mathrm{t}} \sin \beta_{k}^{\prime}+l_{\mathrm{s}} \sin \beta_{k}\right) \\
+\left(F_{z}^{\left(f_{k}\right)}-\frac{m_{\mathrm{t}} g}{2}-m_{\mathrm{s}} g\right) l_{\mathrm{t}} \cos \beta_{k}^{\prime}+\left(F_{z}^{\left(f_{k}\right)}-\frac{m_{\mathrm{s}} g}{2}\right) l_{\mathrm{s}} \cos \beta_{k}, & \leq 1286 \mathrm{~N} \cdot \mathrm{m} \\
-1286 \mathrm{~N} \cdot \mathrm{m} & \leq F_{x}^{\left(f_{k}\right)} l_{\mathrm{s}} \sin \beta_{k}+\left(F_{z}^{\left(f_{k}\right)}-\frac{1}{2} m_{\mathrm{s}} g\right) l_{\mathrm{s}} \cos \beta_{k} \\
\leq & 1286 \mathrm{~N} \cdot \mathrm{m}
\end{aligned}\right.
$$

When the leg $s_{k}$ is in full extension, its maximum projection length is $l_{\mathrm{c}}+l_{\mathrm{t}}+l_{\mathrm{s}}$. According to Equation (59), it can be concluded that the maximum value of $F_{y}^{\left(f_{k}\right)}$ is 525 $\mathrm{N} \cdot \mathrm{m}$ and it is much greater than the value of actual working condition. Hence, the torque of abductor joint does not need to be restrained. Based on Equations (30), (32), (34), (55), and (59), the constraint conditions of driving joints can be written for the legs 2,4 , and 6 . Then

$$
\begin{aligned}
& -1286 \mathrm{~N} \cdot \mathrm{m} \leq-\frac{{ }^{\mathrm{B}} F_{x}^{\left(s_{2}\right)}\left(l_{\mathrm{t}} \sin \beta_{2}^{\prime}+l_{\mathrm{s}} \sin \beta_{2}\right)}{\cos \theta_{2}} \\
& +\left(\left(m_{\mathrm{L}} g+m_{\mathrm{R}} g\right) / 3-m_{\mathrm{s}} g / 2\right) l_{\mathrm{s}} \cos \beta_{2} \\
& +\left(\left(m_{\mathrm{L}} g+m_{\mathrm{R}} g\right) / 3-m_{\mathrm{t}} g / 2-m_{\mathrm{s}} g\right) l_{\mathrm{t}} \cos \beta_{2}^{\prime} \\
& \leq 1286 \mathrm{~N} \cdot \mathrm{m} \\
& -1286 \mathrm{~N} \cdot \mathrm{m} \leq-\frac{{ }^{\mathrm{B}} F_{x}^{\left(s_{2}\right)} l_{\mathrm{s}} \sin \beta_{2}}{\cos \theta_{2}} \\
& +\left(\left(m_{\mathrm{L}} g+m_{\mathrm{R}} g\right) / 3-m_{\mathrm{s}} g / 2\right) l_{\mathrm{s}} \cos \beta_{2} \\
& \leq 1286 \mathrm{~N} \cdot \mathrm{m} \\
& \left\{\begin{array}{c}
-1286 \mathrm{~N} \cdot \mathrm{m} \leq \frac{{ }^{\mathrm{B}} F_{x}^{\left(s_{4}\right)}\left(l_{\mathrm{t}} \sin \beta_{4}^{\prime}+l_{\mathrm{s}} \sin \beta_{4}\right)}{\sin \left(30^{\circ}+\theta_{4}\right)} \\
+\left(\left(m_{\mathrm{L}} g+m_{\mathrm{R}} g\right) / 3-m_{\mathrm{s}} g / 2\right) l_{\mathrm{s}} \cos \beta_{4} \\
+\left(\left(m_{\mathrm{L}} g+m_{\mathrm{R}} g\right) / 3-m_{\mathrm{t}} g / 2-m_{\mathrm{s}} g\right) l_{\mathrm{t}} \cos \beta_{4}^{\prime} \\
\leq 1286 \mathrm{~N} \cdot \mathrm{m} \\
-1286 \mathrm{~N} \cdot \mathrm{m} \leq \frac{{ }^{\mathrm{B}} F_{x}^{\left(s_{4}\right)} l_{\mathrm{s}} \sin \beta_{4}}{\sin \left(30^{\circ}+\theta_{4}\right)} \\
+\left(\left(m_{\mathrm{L}} g+m_{\mathrm{R}} g\right) / 3-m_{\mathrm{s}} g / 2\right) l_{\mathrm{s}} \cos \beta_{4} \\
\leq 1286 \mathrm{~N} \cdot \mathrm{m}
\end{array},\right.
\end{aligned}
$$




$$
\left\{\begin{array}{rl}
-1286 \mathrm{~N} \cdot \mathrm{m} & \leq{ }^{\mathrm{B}} F_{x}^{\left(s_{6}\right)}\left(l_{\mathrm{t}} \sin \beta_{6}^{\prime}+l_{\mathrm{s}} \sin \beta_{6}\right) / \sin \left(30^{\circ}-\theta_{6}\right) \\
+ & \left(\left(m_{\mathrm{L}} g+m_{\mathrm{R}} g\right) / 3-m_{\mathrm{s}} g / 2\right) l_{\mathrm{s}} \cos \beta_{6} \\
+ & \left(\left(m_{\mathrm{L}} g+m_{\mathrm{R}} g\right) / 3-m_{\mathrm{t}} g / 2-m_{\mathrm{s}} g\right) l_{\mathrm{t}} \cos \beta_{6}^{\prime} \\
\leq & 1286 \mathrm{~N} \cdot \mathrm{m} \\
-1286 \mathrm{~N} \cdot \mathrm{m} & \leq{ }^{\mathrm{B}} F_{x}^{\left(s_{6}\right)} l_{\mathrm{s}} \sin \beta_{6} / \sin \left(30^{\circ}-\theta_{6}\right) \\
+ & \left(\left(m_{\mathrm{L}} g+m_{\mathrm{R}} g\right) / 3-m_{\mathrm{s}} g / 2\right) l_{\mathrm{s}} \cos \beta_{6} \\
& \leq 1286 \mathrm{~N} \cdot \mathrm{m}
\end{array} .\right.
$$

\subsection{Equality Constraint Conditions}

\subsubsection{Constraint Condition of Body Height}

To maintain the high load capacity of robot on the ground, the bottom of body runs parallel to the ground when the robot walks. The position vector in the $z$ direction for the foot end of the support leg $s_{k}$ is consistent with the body height $h$ under the body coordinate system $\sum_{\mathrm{B}}$. Then

$$
{ }^{\mathrm{B}} P_{\mathrm{F} z}^{\left(s_{k}\right)}=-h,
$$

According to Equation (63), the body height $h$ can be expressed by the legs 2,4 , and 6 in the body coordinate system $\sum_{\mathrm{B}}$. Then

$$
h=l_{t} \sin \beta_{i}^{\prime}+l_{s} \sin \beta_{i} \quad(i=2,4,6) .
$$

\subsubsection{Constraint Condition of Equal Foot Force}

Due to no external forces in the walking process of robot, the equilibrium constraint conditions of foot forces need to be met for the support legs under the tripod gait.

Then

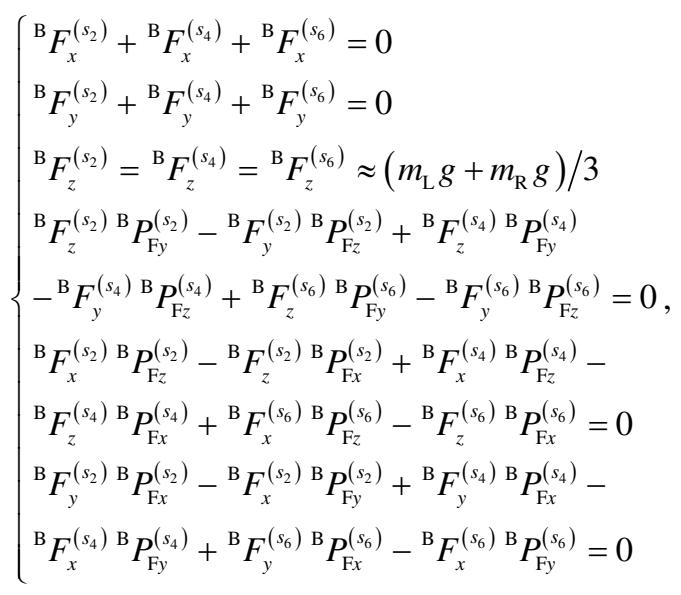

According to Equation (65), the constraints of foot forces in the $x$ direction can be written among the legs 2, 4, and 6 . Then

$$
\left\{\begin{array}{l}
{ }^{\mathrm{B}} F_{x}^{\left(s_{2}\right)}=-\frac{\cot \left(30^{\circ}-\theta_{6}\right)+\cot \left(30^{\circ}+\theta_{4}\right)}{\tan \theta_{2}+\cot \left(30^{\circ}+\theta_{4}\right)}{ }^{\mathrm{B}} F_{x}^{\left(s_{6}\right)} \\
{ }^{\mathrm{B}} F_{x}^{\left(s_{4}\right)}=\frac{\cot \left(30^{\circ}-\theta_{6}\right)-\tan \theta_{2}}{\tan \theta_{2}+\cot \left(30^{\circ}+\theta_{4}\right)}{ }^{\left(s_{6}\right)}
\end{array}\right.
$$

Based on Equation (66), $-8.7^{\circ} \leq \theta_{2} \leq 0^{\circ},-3.8^{\circ} \leq \theta_{4} \leq 0^{\circ}$, and $-5^{\circ} \leq \theta_{6} \leq 0^{\circ}$, the Equation (56) can be rewritten as follows. Then

$$
\left|{ }^{\mathrm{B}} F_{x}^{\left(s_{6}\right)}\right| \leq \frac{1}{3}\left(m_{\mathrm{L}} g+m_{\mathrm{R}} g\right) \mu \frac{\cos \left(30^{\circ}+\theta_{4}-\theta_{2}\right)}{\sin \left(60^{\circ}+\theta_{4}-\theta_{6}\right)} \sin \left(30^{\circ}-\theta_{6}\right),
$$

According to Equation (66), $0^{\circ} \leq \theta_{2} \leq 8.7^{\circ}, 0^{\circ} \leq \theta_{4} \leq 5^{\circ}$, and $0^{\circ} \leq \theta_{6} \leq 3.8^{\circ}$, Equation (56) can also be rewritten as follows. Then

$$
\left|{ }^{\mathrm{B}} F_{x}^{\left(s_{6}\right)}\right| \leq \frac{1}{3}\left(m_{\mathrm{L}} g+m_{\mathrm{R}} g\right) \mu \frac{\cos \left(30^{\circ}+\theta_{4}-\theta_{2}\right)}{\cos \left(30^{\circ}-\theta_{6}-\theta_{2}\right)} \sin \left(30^{\circ}-\theta_{6}\right) .
$$

\section{Power Consumption Solution of Robot Mobile System under Tripod Gait}

According to Equation (52), it can be concluded that the simplified mathematical model has 18 variables for the power consumption of robot mobile system when the robot walks under the tripod gait. Although the range of each variable has been known, the enormous amount of computation will be caused by changing 18 variables together. Hence, the values of partial variables are assumed in their range. The influences of remaining variables are analyzed on the power consumption of robot mobile system.

Based on Equations (52), (66), (67), and (68), it can be concluded that Equation (52) contains only the rotational angle variables, which are $\beta_{2}{ }^{\prime}, \beta_{4}{ }^{\prime}, \beta_{6}{ }^{\prime}, \beta_{1}{ }^{\prime}, \beta_{3}{ }^{\prime}$, and $\beta_{5}{ }^{\prime}$ for the hip joints and $\beta_{2}, \beta_{4}, \beta_{6}, \beta_{1}, \beta_{3}$, and $\beta_{5}$ for the knee joints of robot, when the values of ${ }^{\mathrm{B}} F_{x}^{\left(s_{6}\right)}$ and rotational angles $\theta_{2}, \theta_{4}$, and $\theta_{6}$ of abductor joints have been known. The homogeneous joints have the same ranges of rotational angles for the hip joints and knee joints. To reduce the difficulty of numerical solution in the power consumption of robot mobile system, it can be prescribed that the homogeneous joints have the same rotational angles. Then, Equation (52) includes only the rotational angle $\beta_{i}{ }^{\prime}$ of hip joint and rotational angle $\beta_{i}$ of knee joint, which is propitious to solve the power consumption of robot mobile system under the tripod gait. 
According to the assumed values of variables, the power consumption solution of the robot mobile system can be divided into three first order working conditions; they are the working conditions i, ii and iii. Each of the first order working condition contains three secondary working conditions. Meanwhile, the first order working conditions i, ii, and iii respectively correspond to the left boundary of interval, zero value, and right boundary of interval for the rotational angles of abductor joints. The secondary working conditions $\mathrm{i}-1$, ii-1, and iii- 1 correspond to the maximum constraint positive values for the $x$ directional forces of leg 6 under the corresponding first order working conditions. The secondary working conditions i-2, ii-2, and iii- 2 correspond to the maximum constraint negative values for the $x$ directional forces of leg 6 under the corresponding first order working conditions. The secondary working conditions i-3, ii-3, and iii-3 correspond to the zero values for the $x$ directional foot forces of leg 6 under the corresponding first order working conditions. Then, the working conditions of variable values for the power consumption solution of robot mobile system are respectively shown in Table 2.

Table 2 Working conditions of variable values

\begin{tabular}{|c|c|c|c|}
\hline \multicolumn{2}{|c|}{ Working conditions } & \multirow{2}{*}{$\begin{array}{l}\text { Rotational angles of abductor joints under support phase } /\left({ }^{\circ}\right) \\
\theta_{2}=-8.7^{\circ}, \theta_{4}=-3.8^{\circ}, \theta_{6}=-5^{\circ}\end{array}$} & \multirow{2}{*}{$\frac{X \text { directional foot forces }{ }^{\mathrm{B}} F_{x}^{\left(s_{6}\right)} \text { of leg } 6 /(\mathrm{N})}{{ }^{\mathrm{B}} F_{x_{-} \max }^{\left(s_{s}\right)}=0.1789\left(m_{\mathrm{L}} g+m_{\mathrm{R}} g\right) \mu}$} \\
\hline \multirow{3}{*}{ i } & $\mathrm{i}-1$ & & \\
\hline & $\mathrm{i}-2$ & $\theta_{2}=-8.7^{\mathrm{o}}, \theta_{4}=-3.8^{\circ}, \theta_{6}=-5^{\mathrm{o}}$ & ${ }^{\mathrm{B}} F_{x_{-} \max }^{\left(s_{6}\right)}=-0.1789\left(m_{\mathrm{L}} g+m_{\mathrm{R}} g\right) \mu$ \\
\hline & $\mathrm{i}-3$ & $\theta_{2}=-8.7^{\mathrm{o}}, \theta_{4}=-3.8^{\circ}, \theta_{6}=-5^{\circ}$ & ${ }^{\mathrm{B}} F_{x_{-} \max }^{\left(s_{6}\right)}=0$ \\
\hline \multirow{3}{*}{ ii } & ii-1 & $\theta_{2}=\theta_{4}=\theta_{6}=0^{\circ}$ & ${ }^{\mathrm{B}} F_{x_{-} \max }^{\left(s_{6}\right)}=0.1667\left(m_{\mathrm{L}} g+m_{\mathrm{R}} g\right) \mu$ \\
\hline & ii-2 & $\theta_{2}=\theta_{4}=\theta_{6}=0^{\circ}$ & ${ }^{\mathrm{B}} F_{x_{-} \max }^{\left(s_{s}\right)}=-0.1667\left(m_{\mathrm{L}} g+m_{\mathrm{R}} g\right) \mu$ \\
\hline & ii-3 & $\theta_{2}=\theta_{4}=\theta_{6}=0^{\circ}$ & ${ }^{\mathrm{B}} F_{x_{-} \max }^{\left(s_{6}\right)}=0$ \\
\hline \multirow{3}{*}{ iii } & iii-1 & $\theta_{2}=8.7^{\circ}, \theta_{4}=5^{\circ}, \theta_{6}=3.8^{\circ}$ & ${ }^{\mathrm{B}} F_{x_{-} \max }^{\left(s_{6}\right)}=0.1383\left(m_{\mathrm{L}} g+m_{\mathrm{R}} g\right) \mu$ \\
\hline & iii-2 & $\theta_{2}=8.7^{\circ}, \theta_{4}=5^{\circ}, \theta_{6}=3.8^{\circ}$ & ${ }^{\mathrm{B}} F_{x_{-} \max }^{\left(s_{s}\right)}=-0.1383\left(m_{\mathrm{L}} g+m_{\mathrm{R}} g\right) \mu$ \\
\hline & iii-3 & $\theta_{2}=8.7^{\circ}, \theta_{4}=5^{\circ}, \theta_{6}=3.8^{\circ}$ & ${ }^{\mathrm{B}} F_{x_{-} \max }^{\left(s_{6}\right)}=0$ \\
\hline
\end{tabular}

Then, the mass of the large-load-ratio six-legged robot is presumed to be $300 \mathrm{~kg}$. The maximum mass of carrying materials is $150 \mathrm{~kg}$. The effective dimensions of the robot are about $1.2 \mathrm{~m} \times 1.2 \mathrm{~m} \times 1.0 \mathrm{~m}$. The MATLAB software is used to carry out the numerical solution for the power consumption $P_{\text {sum }}$ of robot mobile system based on the size and quality parameters of the robot, results of above simplification analysis, constraint conditions of stable walking. The rotational angles of hip joint and knee joint can change within their own reachable ranges. The range of body height $h$ is between $0 \mathrm{~m}$ and $1 \mathrm{~m}$. The range of span $L_{\mathrm{p} i}$ is between $0 \mathrm{~m}$ and $1.18 \mathrm{~m}$. The normal forces of the foot ends are $\left(m_{\mathrm{L}} \mathrm{g}+m_{\mathrm{R}} \mathrm{g}\right) / 3$ for the support legs 2,4 , and 6.

For the convenience of following analysis, the variable tendency charts of the power consumption $P_{\text {sum }}$ of robot mobile system can be composed of chart fields $x_{i-j}$. Where $x$ is $A, B, C, D, E$, and $F . i$ is i, ii, and iii. $j$ is 1,2 , and 3 .

\subsection{Numerical Solution of Power Consumption under Left Boundary of Rotational Angle of Abductor Joint}

The foot ends of support legs 2, 4, and 6 are specified at the point $s_{\mathrm{d}}$. Then, the values of rotational angles $\theta_{2}, \theta_{4}$, and $\theta_{6}$ are $-8.7^{\circ},-3.8^{\circ}$, and $-5^{\circ}$ for the abductor joints of legs 2,4 , and 6 , respectively. The foot force of leg 6 in the $x$ direction is constrained.

Then

$$
\left|{ }^{\mathrm{B}} F_{x}^{\left(s_{6}\right)}\right| \leq 0.1789\left(m_{\mathrm{L}} g+m_{\mathrm{R}} g\right) .
$$

The variable tendency charts of the power consumption $P_{\text {sum }}$ of robot mobile system are respectively gained under the secondary working conditions i-1, i-2, and i-3; they are shown in Figures 8, 9, and 10.

Based on Figures 8, 9, and 10, it can be concluded that the large-load-ratio six-legged robot, which walks by the mixture-type II tripod gait under the first order working conditions i, can have lower power consumption $P_{\text {sum }}$ of mobile system when the rotational angle $\beta$ of knee joint should be between $107.5^{\circ}$ and $150^{\circ}$, the rotational angle $\beta^{\prime}$ of hip joint should be taken near the boundary of desirable interval from $-72^{\circ}$ to $72^{\circ}$, and the span $L_{\mathrm{p}}$ is in the range of $0.18 \mathrm{~m}$ to $0.55 \mathrm{~m}$. To make the large-load-ratio six-legged robot more flexible, the left boundary of the rotational angle of abductor joint can be relaxed to $72.5^{\circ}$ for the rotational angle $\beta$ of knee joint. 

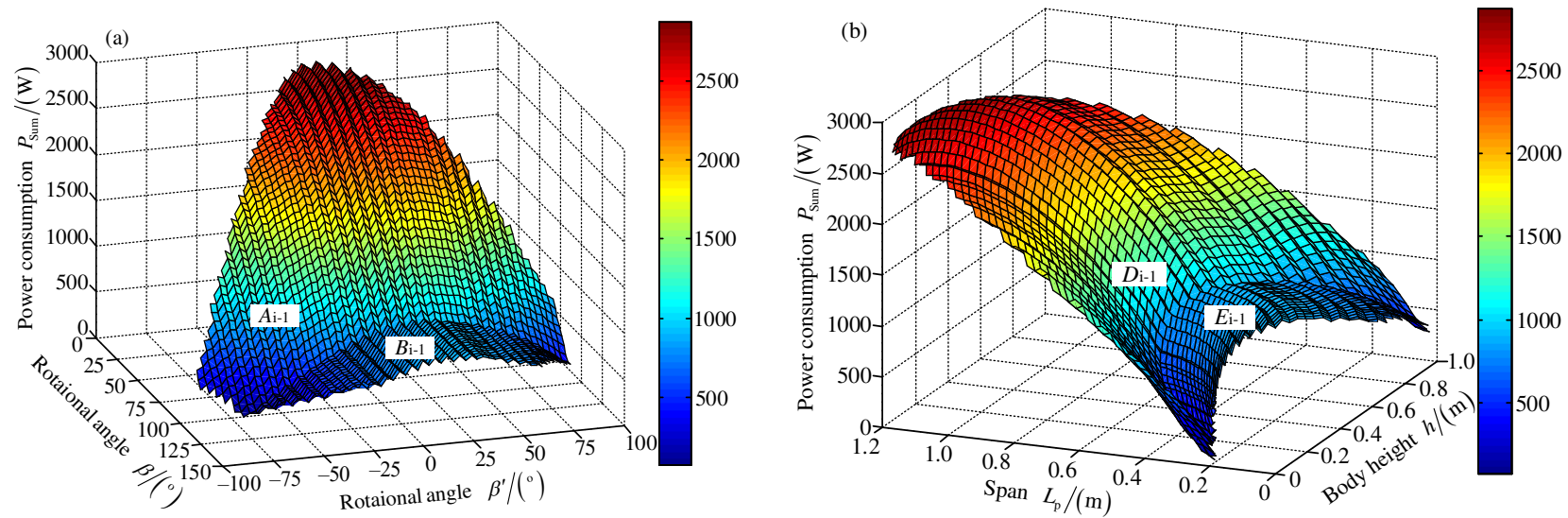

Figure 8 Variable tendency charts of $P_{\text {sum }}$ under secondary working conditions i-1: (a) with changes in $\beta^{\prime}$ and $\beta$; (b) with changes in $h$ and $L_{\mathrm{p}}$
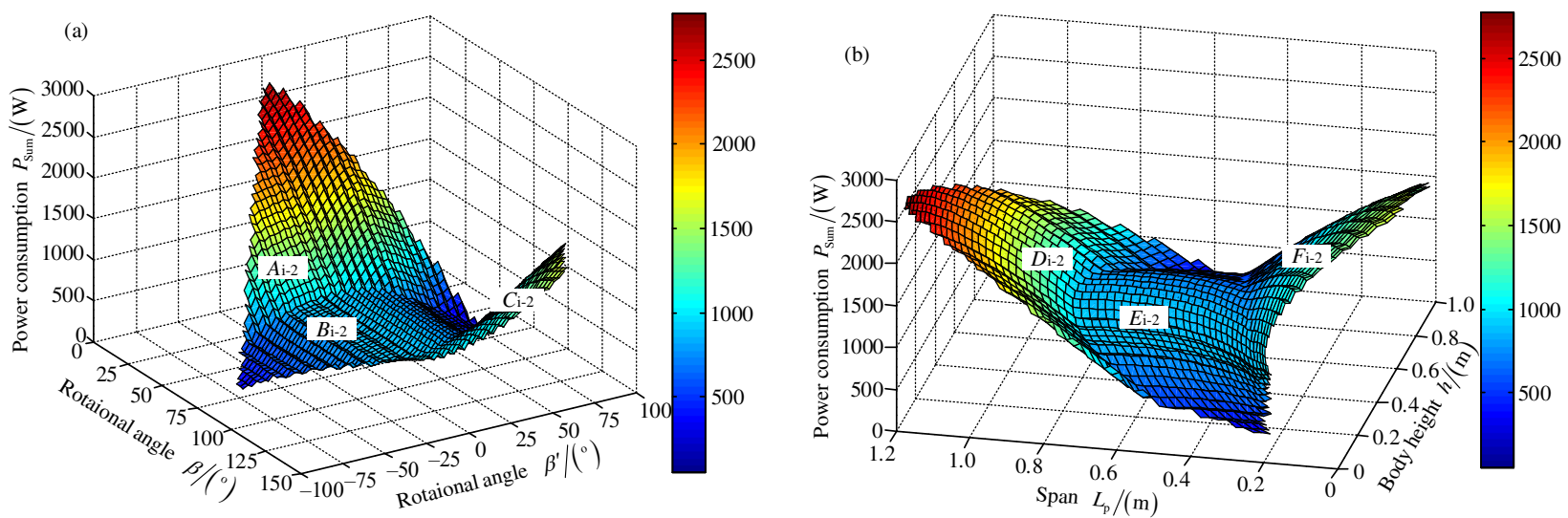

Figure 9 Variable tendency charts of $P_{\text {sum }}$ under secondary working conditions i-2: (a) with changes in $\beta^{\prime}$ and $\beta$; (b) with changes in $h$ and $L_{\mathrm{p}}$
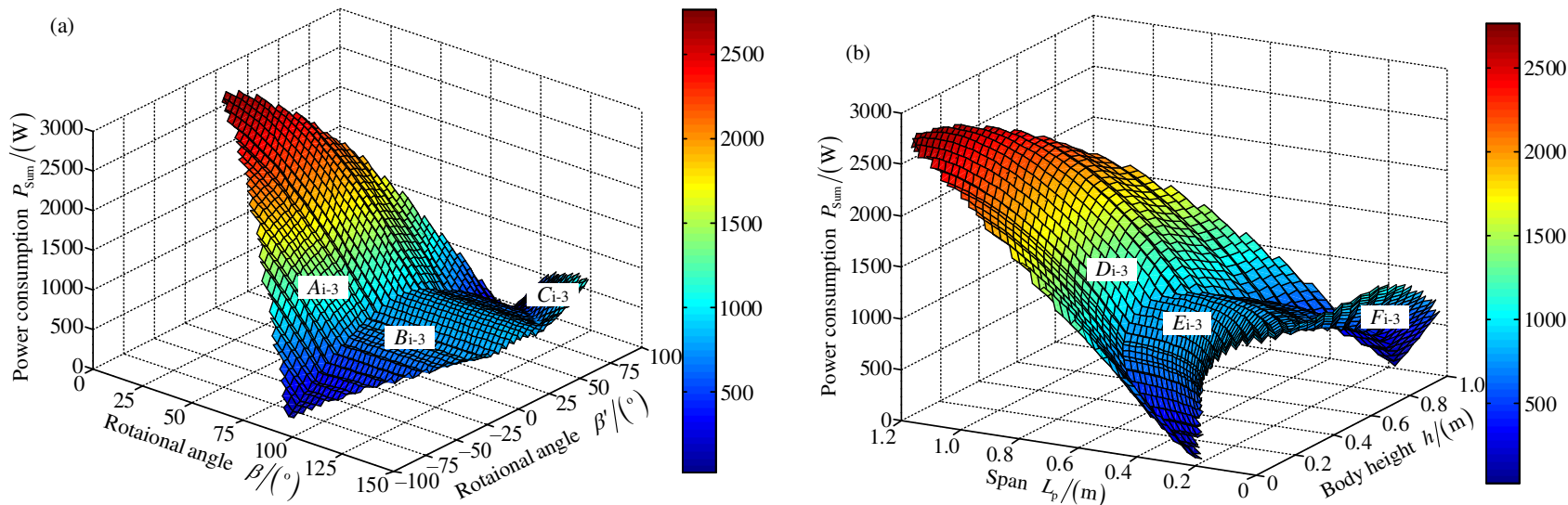

Figure 10 Variable tendency charts of $P_{\text {sum }}$ under secondary working conditions i-3: (a) with changes in $\beta^{\prime}$ and $\beta$; (b) with changes in $h$ and $L_{\mathrm{p}}$

\subsection{Numerical Solution of Power Consumption under Zero Value of Rotational Angle of Abductor Joint}

The foot ends of support legs 2, 4, and 6 are specified at the middle position between the point $s_{\mathrm{u}}$ and the point $s_{\mathrm{d}}$. Then, the values of rotational angles $\theta_{2}, \theta_{4}$, and $\theta_{6}$ are $0^{\circ}$ for the abductor joints of legs 2,4 , and 6 . The foot force of leg 6 in the $x$ direction is constrained. Then

$$
\left|{ }^{\mathrm{B}} F_{x}^{\left(s_{6}\right)}\right| \leq 0.1667\left(m_{\mathrm{L}} g+m_{\mathrm{R}} g\right) \mu .
$$


The variable tendency charts of the power consumption $P_{\text {sum }}$ of robot mobile system are respectively gained under the secondary working conditions ii-1, ii-2, and ii-3; they are shown in Figures 11, 12, and 13.
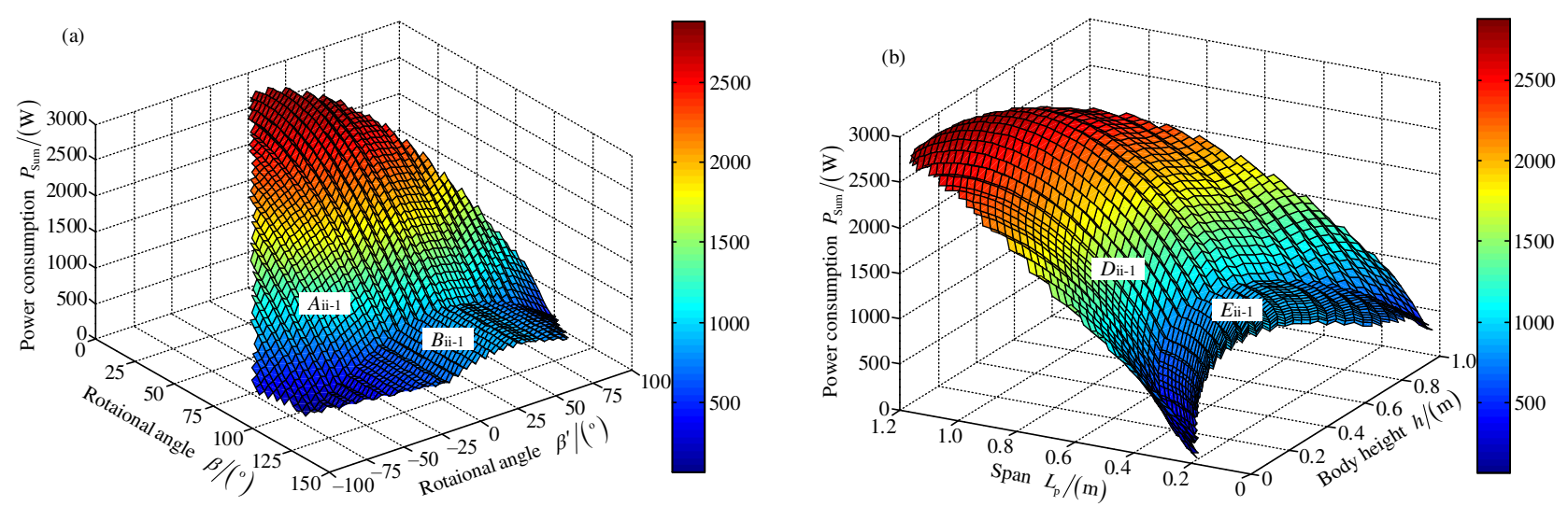

Figure 11 Variable tendency charts of $P_{\text {sum }}$ under secondary working conditions ii-1: (a) with changes in $\beta^{\prime}$ and $\beta$; (b) with changes in $h$ and $L_{\mathrm{p}}$
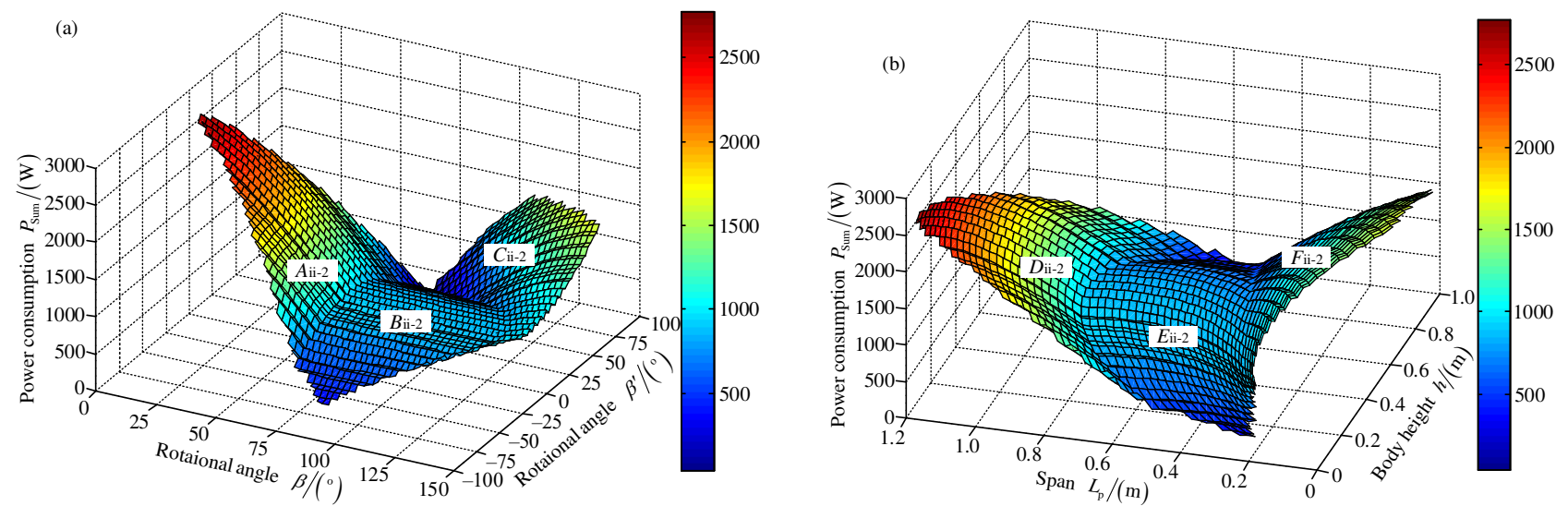

Figure 12 Variable tendency charts of $P_{\text {sum }}$ under secondary working conditions ii-2: (a) with changes in $\beta^{\prime}$ and $\beta$; (b) with changes in $h$ and $L_{\mathrm{p}}$
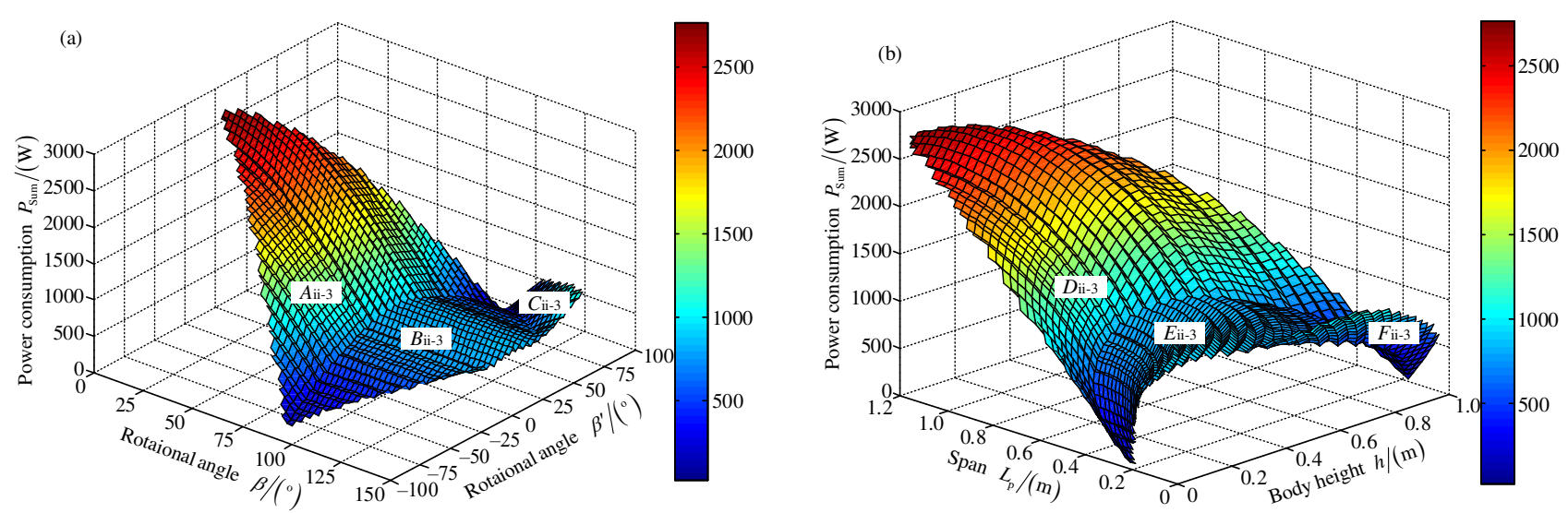

Figure 13 Variable tendency charts of $P_{\text {sum }}$ under secondary working conditions ii-3: (a) with changes in $\beta^{\prime}$ and $\beta$; (b) with changes in $h$ and $L_{\mathrm{p}}$

According to Figures 11, 12, and 13, it can be concluded that the large-load-ratio six-legged robot, which walks by the mixture-type II tripod gait under the first order working conditions ii, can have lower power consumption $P_{\text {sum }}$ of 
mobile system when the rotational angle $\beta$ of knee joint should be between $107.5^{\circ}$ and $150^{\circ}$, the rotational angle $\beta^{\prime}$ of hip joint should be taken near the boundary of desirable interval from $-72^{\circ}$ to $72^{\circ}$, and the span $L_{\mathrm{p}}$ is in the range of $0.18 \mathrm{~m}$ to $0.55 \mathrm{~m}$. To make the large-load-ratio six-legged robot more flexible, the left boundary of the rotational angle of abductor joint can be relaxed to $72.5^{\circ}$ for the rotational angle $\beta$ of knee joint.

\subsection{Numerical Solution of Power Consumption under} Right Boundary of Rotational Angle of Abductor Joint

The foot ends of support legs 2, 4, and 6 are specified at the point $s_{\mathrm{u}}$. Then, the values of rotational angles $\theta_{2}, \theta_{4}$, and $\theta_{6}$ are $8.7^{\circ}, 5^{\circ}$, and $3.8^{\circ}$ for the abductor joints of legs 2 , 4 , and 6 , respectively. The foot force of leg 6 in the $x$ direction is constrained.

Then

$$
\left|{ }^{\mathrm{B}} F_{x}^{\left(s_{6}\right)}\right| \leq 0.1383\left(m_{\mathrm{L}} g+m_{\mathrm{R}} g\right) \mu .
$$

The variable tendency charts of the power consumption $P_{\text {sum }}$ of robot mobile system are respectively gained under the secondary working conditions iii-1, iii-2, and iii-3; they are shown in Figures 14, 15, and 16.

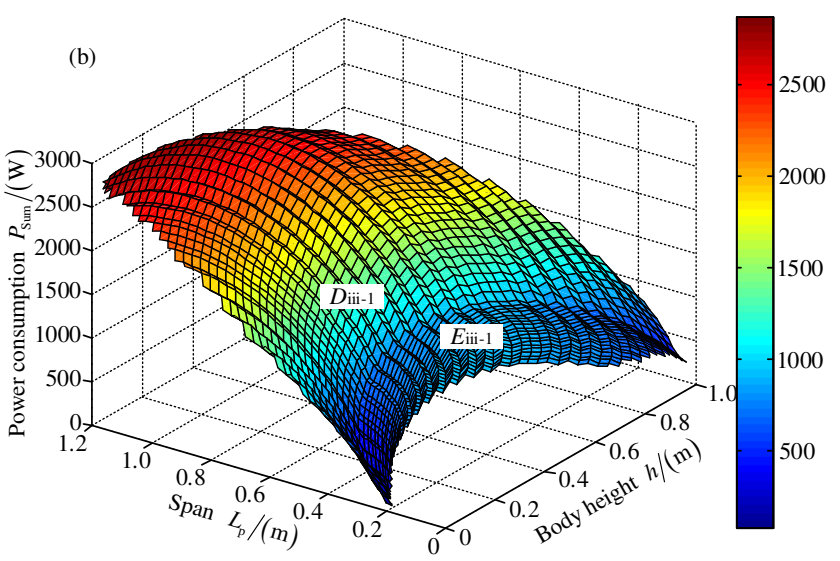

Figure 14 Variable tendency charts of $P_{\text {sum }}$ under secondary working conditions iii-1: (a) with changes in $\beta^{\prime}$ and $\beta$; (b) with changes in $h$ and $L_{\mathrm{p}}$
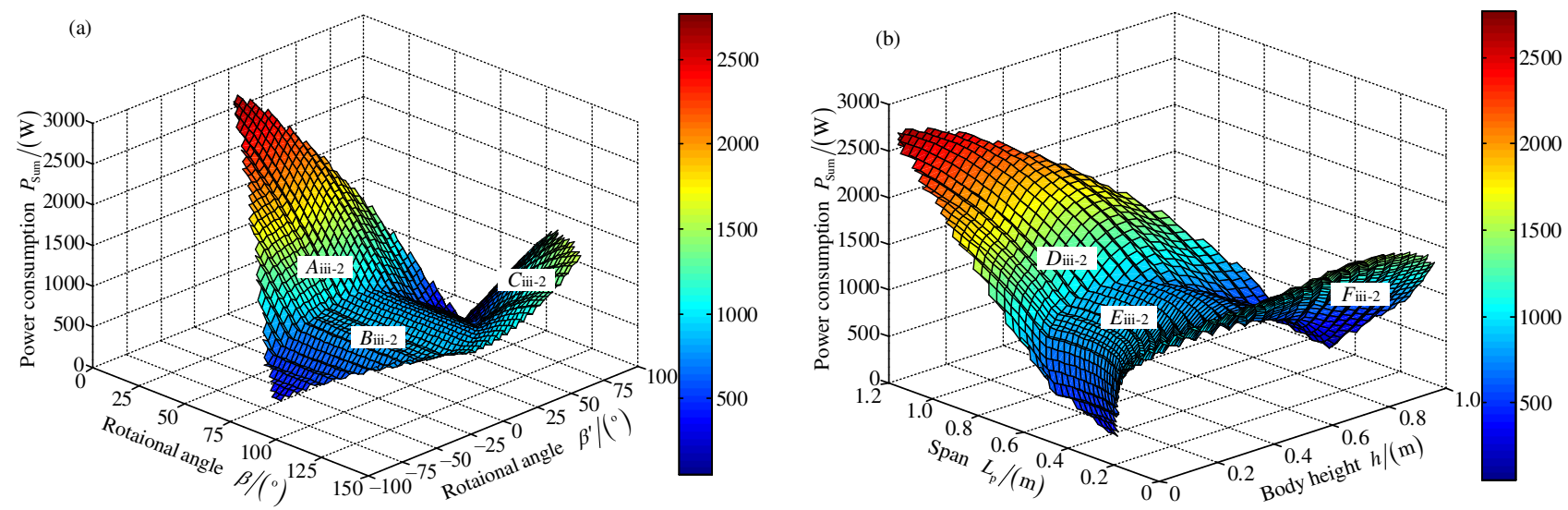

Figure 15 Variable tendency charts of $P_{\text {sum }}$ under secondary working conditions iii-2: (a) with changes in $\beta^{\prime}$ and $\beta$; (b) with changes in $h$ and $L_{\mathrm{p}}$

Based on Figures 14, 15, and 16, it can be concluded the large-load-ratio six-legged robot, which walks by the mixture-type II tripod gait under the first order working conditions iii, can have lower power consumption $P_{\text {sum }}$ of mobile system when the rotational angle $\beta$ of knee joint should be between $105^{\circ}$ and $150^{\circ}$, the rotational angle $\beta^{\prime}$ of hip joint should be taken near the boundary of desirable interval from $-72^{\circ}$ to $72^{\circ}$, and the span $L_{\mathrm{p}}$ is in the range of $0.18 \mathrm{~m}$ to $0.55 \mathrm{~m}$. To make the large-load-ratio six-legged robot more flexible, the left boundary of the rotational angle of abductor joint can be relaxed to $75^{\circ}$ for the rotational angle $\beta$ of knee joint. 

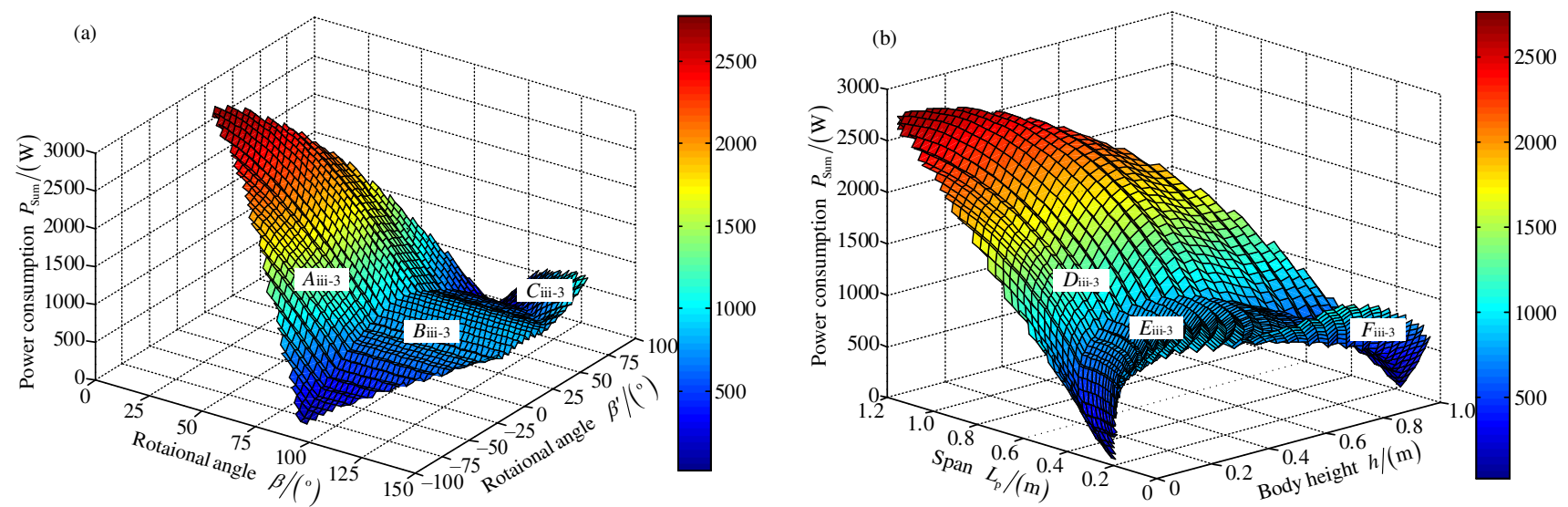

Figure 16 Variable tendency charts of $P_{\text {sum }}$ under secondary working conditions iii-3: (a) with changes in $\beta^{\prime}$ and $\beta$; (b) with changes in $h$ and $L_{\mathrm{p}}$

\subsection{Solving Results of Power Consumption of Robot Mobile System under Tripod Gait}

Based on Equation (52), the power consumption of robot mobile system under the mixture-type II tripod gait is solved numerically. The analysis results are obtained as follows.

(1) When the rotational angles of abductor joints are within the prescribed ranges $\left(-8.7^{\circ} \leq \theta_{2} \leq 8.7^{\circ},-3.8^{\circ} \leq \theta_{4} \leq 5^{\circ}\right.$, $\left.-5^{\circ} \leq \theta_{6} \leq 3.8^{\circ}\right)$ for the legs 2,4 , and 6 , the normal foot forces of legs 2, 4, and 6 are about $1 / 3$ of the sum of robot weight and the load. The changes of $\theta_{2}, \theta_{4}$, and $\theta_{6}$ within the prescribed range have little effect on the power consumption $P_{\text {sum }}$ of robot mobile system.
(2) The value of ${ }^{\mathrm{B}} F_{x_{-} \text {max }}^{\left(s_{6}\right)}$ affects the values of ${ }^{\mathrm{B}} F_{x_{-} \max }^{\left(s_{2}\right)}$ and ${ }^{\mathrm{B}} F_{x x_{\max }}^{\left(s_{4}\right)}$. The changes of ${ }^{\mathrm{B}} F_{x_{-} \max }^{\left(s_{2}\right)},{ }^{\mathrm{B}} F_{x_{-} \max }^{\left(s_{4}\right)}$ and ${ }^{\mathrm{B}} F_{x_{-} \max }^{\left(s_{6}\right)}$ have some influence on the power consumption $P_{\text {sum }}$ of robot mobile system, but that influence is not significant.

(3) The chart field $B_{i-j}(i=\mathrm{i}$, ii, iii; $j=1,2$, and 3$)$ corresponds to the lower power consumption $P_{\text {sum }}$ of robot mobile system, which involves the preferred ranges for the rotational joint $\beta^{\prime}$ of hip joint and the rotational joint $\beta$ of knee joint. The whole ranges of rotational angles $\beta^{\prime}$ and $\beta$ which have the lower power consumption $P_{\text {sum }}$ under the working conditions i, ii and iii are respectively shown in Table 3.

Table 3 Whole ranges of rotational angles of $\beta^{\prime}$ and $\beta$ under chart field $B_{\text {i-j }}$ of lower power consumption $P_{\text {sum }}$

\begin{tabular}{|c|c|c|c|c|c|}
\hline \multicolumn{2}{|c|}{ Working conditions } & \multirow{2}{*}{$\frac{\text { Range of } \beta^{\prime}}{-72^{\circ}-90^{\circ}}$} & \multirow{2}{*}{$\frac{\text { Range of } \beta}{107.5^{\circ}-150^{\circ}}$} & \multirow{2}{*}{$\begin{array}{l}\text { Value of } \beta^{\prime} \text { under maximum } P_{\text {sum }} \\
12^{\circ}\end{array}$} & \multirow{2}{*}{$\begin{array}{l}\text { Value of } \beta^{\prime} \text { under minimum } P_{\text {sun }} \\
-72^{\circ}\end{array}$} \\
\hline & $\mathrm{i}-1$ & & & & \\
\hline \multirow[t]{3}{*}{$\mathrm{i}$} & $\mathrm{i}-2$ & $-72^{\circ}-72^{\circ}$ & $72.5^{\circ}-150^{\circ}$ & $-12^{\circ}$ & $72^{\circ}$ \\
\hline & $\mathrm{i}-3$ & $-87^{\circ}-87^{\circ}$ & $90^{\circ}-150^{\circ}$ & $0^{\circ}$ & $-87^{\circ}$ and $87^{\circ}$ \\
\hline & ii -1 & $-72^{\circ}-90^{\circ}$ & $107.5^{\circ}-150^{\circ}$ & $12^{\circ}$ & $-72^{\circ}$ \\
\hline \multirow[t]{3}{*}{ ii } & ii-2 & $-72^{\circ}-72^{\circ}$ & $72.5^{\circ}-150^{\circ}$ & $-12^{\circ}$ & $72^{\circ}$ \\
\hline & ii-3 & $-87^{\circ}-87^{\circ}$ & $90^{\circ}-150^{\circ}$ & $0^{\circ}$ & $-87^{\circ}$ and $87^{\circ}$ \\
\hline & iii-1 & $-72^{\circ}-90^{\circ}$ & $105^{\circ}-150^{\circ}$ & $12^{\circ}$ & $-72^{\circ}$ \\
\hline \multirow[t]{2}{*}{ iii } & iii-2 & $-72^{\circ}-72^{\circ}$ & $75^{\circ}-150^{\circ}$ & $-12^{\circ}$ & $72^{\circ}$ \\
\hline & iii-3 & $-87^{\circ}-87^{\circ}$ & $90^{\circ}-150^{\circ}$ & $0^{\circ}$ & $-87^{\circ}$ and $87^{\circ}$ \\
\hline
\end{tabular}

According to Table 3, it can be concluded that the large-load-ratio six-legged robot has relatively lower power consumption of mobile system when the ranges of the rotational angles of abductor joints should be $-8.7^{\circ} \leq \theta_{2} \leq 8.7^{\circ},-3.8^{\circ} \leq \theta_{4} \leq 5^{\circ}$, and $-5^{\circ} \leq \theta_{6} \leq 3.8^{\circ}$, the optimal ranges of the rotational angle of hip joint should be $-72^{\circ} \leq \beta^{\prime} \leq-12^{\circ}$ and $12^{\circ} \leq \beta^{\prime} \leq 72^{\circ}$, and the optimal ranges of the rotational angle of knee joint should be $107.5^{\circ} \leq \beta \leq 150^{\circ}$. Based on the trafficability, stability and flexibility of robot, the optimal range can be broadened to $72.5^{\circ} \leq \beta \leq 150^{\circ}$ for the rotational angle of knee joint. The rotational angles of hip joint and knee joint do not simultaneously take place at 
the value boundary because of the interference, stability, etc.

(4) The variable trend of the power consumption $P_{\text {sum }}$ moves gradually from augmentation to a decrease when the rotational angle $\beta$ of knee joint is any constant in its interval, and the rotational angle $\beta^{\prime}$ of hip joint varies over its range. Based on Table 3, the value of $\beta^{\prime}$ under the maximum $P_{\text {sum }}$ can be obtained in the chart field $B_{i-j}$. The variable tendencies of $P_{\text {sum }}$ move gradually from augmentation to a decrease with the change in the body height $h$ from $0 \mathrm{~m}$ to $1 \mathrm{~m}$. $P_{\text {sum }}$ usually has the maximum value when the body height $h$ is near $0.58 \mathrm{~m}$. The variable tendency chart of $P_{\text {sum }}$ with the change in the body height $h$ is shown in Figure 17. In Figure 17, $P_{\text {sum }}$ has multiple solutions at the same body height $h$, which is due to the changes of the rotational angle $\beta^{\prime}$ of hip joint and rotational angle $\beta$ of knee joint in their interval. The curve concentrated area is the preferred low power consumption region. To obtain relatively low $P_{\text {sum }}$, the body height $h$ of robot should avoid $0.58 \mathrm{~m}$.

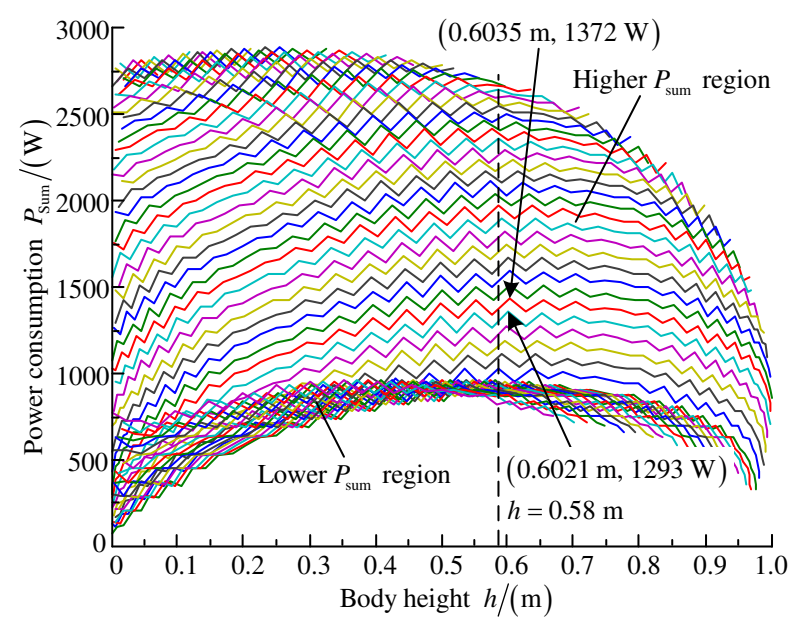

Figure 17 Variable tendency chart of $P_{\text {sum }}$ with change in body height $h$

(5) Based on the stability of robot, the power consumption $P_{\text {sum }}$ of robot mobile system is often relatively low when the span $L_{\mathrm{p}}$ is between $0.18 \mathrm{~m}$ and $0.55 \mathrm{~m}$. The variable tendency chart of $P_{\text {sum }}$ with the change in the span $L_{\mathrm{p}}$ is shown in Figure 18. In Figure 18, $P_{\text {sum }}$ has multiple solutions at the same span $L_{\mathrm{p}}$, which is due to the changes of the rotational angle $\beta^{\prime}$ of hip joint and rotational angle $\beta$ of knee joint in their interval. The curve concentrated area is the preferred low power consumption region. To obtain relatively low $P_{\text {sum, the }}$ body height $h$ of robot should avoid $0.58 \mathrm{~m}$. Then, the overall range of span $L_{\mathrm{p}}$ can be obtained at relatively low $P_{\text {sum. }}$.

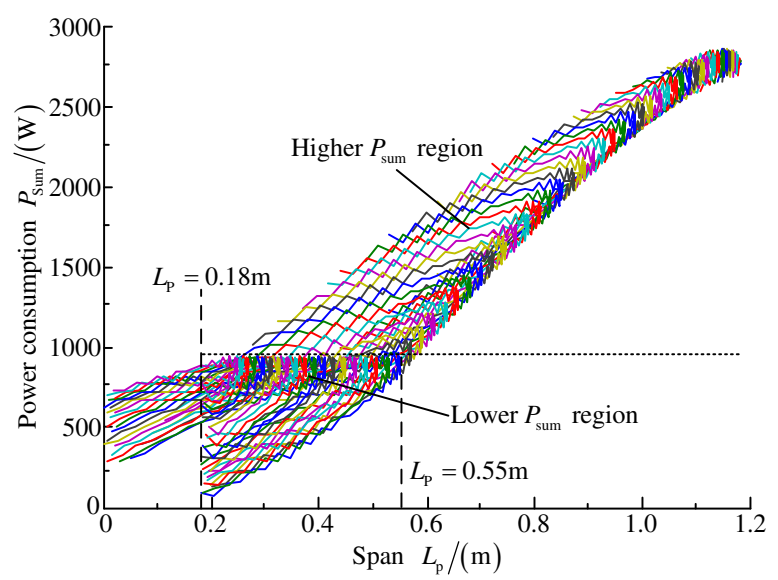

Figure 18 Variable tendency chart of $P_{\text {sum }}$ with change in span $L_{\mathrm{p}}$

\section{Experimental Analysis of Average Power Consumption of Robot Mobile System under Mixture-Type II Tripod Gait}

According to the typical walking modes of the large-load-ratio six-legged robot, the hard ground walking experiments of prototype are implemented with the changes in the duty ratio $\beta_{\mathrm{R}}$, body height $h$ and step pitch $s$. The walking experiments under the mixture-type II tripod gait are respectively shown in Figure 19. To obtain the average power consumption $P_{\mathrm{A}}$ of robot mobile system, the prototype of large-load-ratio six-legged robot walks three gait periods. When the prototype of robot is walking on the hard ground, some parameters are set as follows. The initial span $L_{\mathrm{p} i}^{\prime}$ is $0.68 \mathrm{~m}$. The duty ratio $\beta_{\mathrm{R}}$ is respectively set to $1 / 2,2 / 3$, and $5 / 6$. The movement time $(\mathrm{TM})$ is set to $0.3 \mathrm{~s}$ in the programmable multi-axis controller (PMAC). The maximum swing height $h_{\text {T-max }}$ and initial lift height $h_{\mathrm{TB}}$ of the swing leg are respectively set to $0.10 \mathrm{~m}$ and $0.03 \mathrm{~m}$. The mass $m_{\mathrm{R}}$ of carrying materials is 0 $\mathrm{kg}$.

The prototype of large-load-ratio six-legged robot walks along the $Y_{\mathrm{B}}$ direction of the body coordinate system $\sum_{\mathrm{B}}$ under the mixture-type II tripod gait, quadrangular gait, and pentagon gait, respectively. The initial angles of abductor joints are set to $60^{\circ}$ for the legs $1,3,4$ and 6 . The initial angles of $0^{\circ}$ are set for the abductor joints of legs 2 and 5 .

\subsection{Change of Body Height}

When the large-load- ratio six-legged robot walks under the mixture-type II gait, the body height $h$ varies from 0.25 $\mathrm{m}$ to $0.65 \mathrm{~m}$. The step pitch $s$ is kept at $0.30 \mathrm{~m}$. The variation curves of the average power consumption $P_{\mathrm{A}}$ of 
robot mobile system with the change in the body height $h \quad$ are shown in Figure 20.
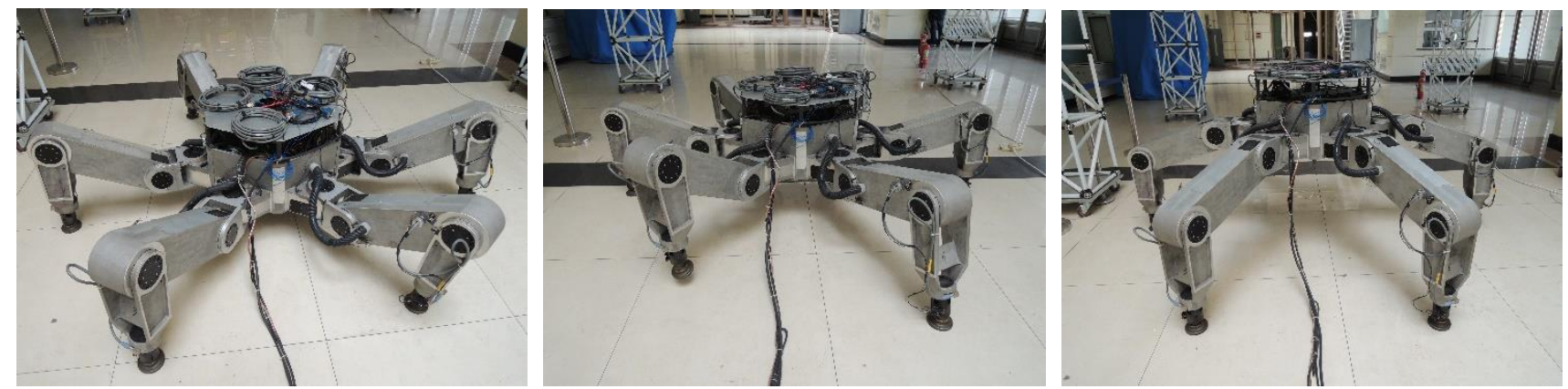

Figure 19 Walking experiments of mixture-type II gait

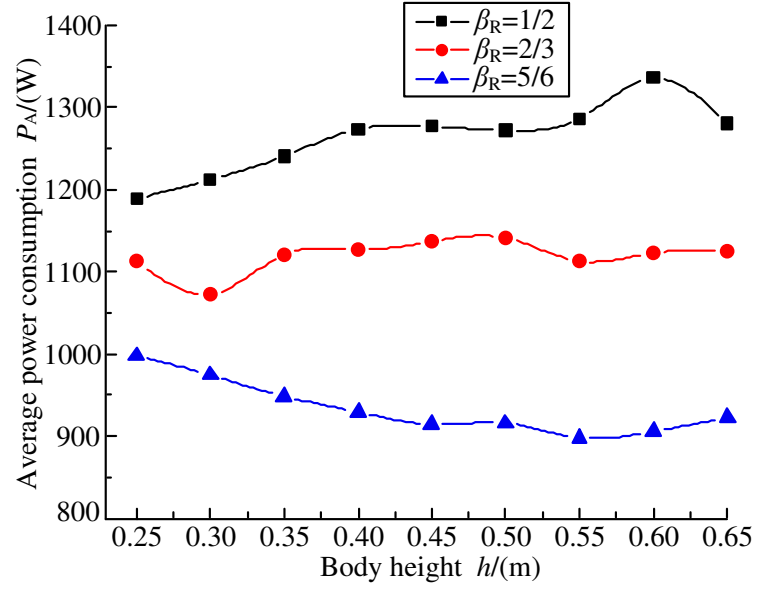

Figure 20 Variation curves of $P_{\mathrm{A}}$ with change in body height $h$

In Figure 20, it shows that the average power consumption of robot mobile system under the mixture-type II tripod gait is the largest among the mixture-type II gait when the body height $h$ changes from $0.25 \mathrm{~m}$ to $0.65 \mathrm{~m}$. The minimum value of the average power consumption $P_{\mathrm{A}}$ can be obtained under the mixture-type II pentagon gait. Based on Figure 20, it is concluded that the average power consumption $P_{\mathrm{A}}$ of robot mobile system is not necessarily minimal when the body height $h$ is the smallest. The average power consumption curves of robot mobile system exhibit the nonlinear variation characteristics under the mixture-type II gait. The corresponding minimum values of the average power consumption can be obtained when the body heights are $0.25 \mathrm{~m}, 0.30 \mathrm{~m}$ and $0.55 \mathrm{~m}$ under the mixture-type II tripod gait, quadrangular gait, and pentagon gait, respectively.

According to Figure 20, it shows that the variable tendency of $P_{\mathrm{A}}$ under the mixture-type II tripod gait moves gradually from augmentation to a decrease with the increase of the body height $h$. The average power consumption $P_{\mathrm{A}}$ of robot mobile system has a maximum value of $1335 \mathrm{~W}$ when the body height is $0.60 \mathrm{~m}$ under the mixture-type II tripod gait. Then, it can be concluded that the experimental results are consistent with the theoretical analysis results of the power consumption of robot mobile system under the tripod gait in section 7 .

Based on Figure 17 in section 7, it can be obtained that the variable tendency of $P_{\text {sum }}$ moves gradually from augmentation to a decrease with the increase of the body height $h$ from $0 \mathrm{~m}$ to $1 \mathrm{~m}$. $P_{\text {sum }}$ usually has the maximum value when the body height $h$ is around $0.58 \mathrm{~m}$. When the body heights are $0.6021 \mathrm{~m}$ and $0.6035 \mathrm{~m}$, the maximum values of $P_{\text {sum }}$ are $1293 \mathrm{~W}$ and $1372 \mathrm{~W}$ respectively. Therefore, according to Figures 17 and 20, it is proved that the theoretical analysis process is reasonable and the analysis results are correct through the experiments of the influences of the gait parameters on the power consumption of robot mobile system. The theoretical analysis results can be applied to the motion planning of the prototype of large-load-ratio six-legged robot so as to keep the relatively low power consumption of mobile system under the tripod gait.

\subsection{Change of Step Pitch}

When the large-load-ratio six-legged robot walks under the mixture-type II gait, the body height $h$ is kept at $0.50 \mathrm{~m}$. The step pitch $s$ varies from $0.10 \mathrm{~m}$ to $0.40 \mathrm{~m}$. The variation curves of the average power consumption $P_{\mathrm{A}}$ of robot mobile system with the change in the step pitch $s$ are shown in Figure 21.

In Figure 21, it shows that the average power consumption of robot mobile system under the mixture-type II tripod gait is the largest among the mixture-type II gait when the step pitch $s$ changes from $0.10 \mathrm{~m}$ to $0.40 \mathrm{~m}$. The minimum value of the average power consumption $P_{\mathrm{A}}$ can be acquired under the mixture-type II pentagon gait. The variable tendency of $P_{\mathrm{A}}$ moves gradually from augmentation to a decrease with the increase of the step pitch $s$ under the mixture-type II tripod 
gait. The average power consumption curve of robot mobile system first decreases, then increases and then decreases under the mixture-type II quadrangular gait. The curve of $P_{\mathrm{A}}$ presents an increasing trend under the mixture-type II pentagon gait with the change in the step pitch $s$. As the step pitch $s$ increases, the average power consumption $P_{\mathrm{A}}$ of robot mobile system does not necessarily increase. Therefore, the robot should choose the corresponding step pitch under the lower power consumption of robot mobile system according to the different modes of walking.

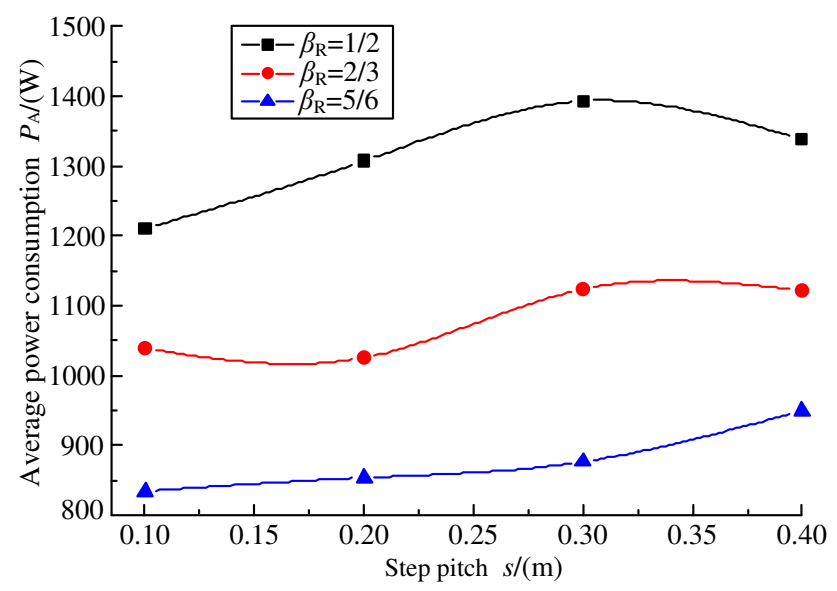

Figure 21 Variation curves of $P_{\mathrm{A}}$ with change in step pitch $s$

To summarize, based on the walking experiments of the prototype of large-load-ratio six-legged robot, the variable tendencies of the average power consumption of robot mobile system are respectively obtained with the changes in the initial angle $\triangle \theta_{i}$ of abductor joint, body height $h$, and step pitch $s$, which is conducive to selecting the suitable gait parameters for the large-load-ratio six-legged robot. The pentagon gait and the tripod gait respectively have the minimum and maximum values of the average power consumption of robot mobile system in terms of the same gait parameters and low power consumption. If the walking speed of robot is taken into account, the tripod gait that has the largest average power consumption of mobile system is often chosen for the prototype of robot. Therefore, the large-load-ratio six-legged robot should choose the appropriate walking gait according to the actual task requirements. The average power consumption of robot mobile system is only one of the factors considered in the gait selecting.

\section{Conclusions and Future Work}

(1) To make the electrically driven large-load-ratio six-legged robot have the characteristics of universal walking, the body of robot adopts a regular polygon. And six legs are evenly distributed around the robot body. Based on the configuration characteristics of robot, four kinds of the typical walking modes are designed; they are the crab type, ant type, mixture type I, and mixture type II.

(2) The dynamics analysis is carried out for the electrically driven large-load-ratio six-legged robot. According to the final output torques of articulated servo motors, the mathematical expressions of joint loads are respectively deduced in the support phase and transfer phase. The mathematical models of the power consumption are established for the single leg and mobile system of the electrically driven large-load-ratio six-legged robot.

(3) The tripod gait is the fastest gait and common gait for the six-legged robot. The power consumption of robot mobile system is studied under the tripod gait. Based on the normal force data of foot ends of the robot prototype during the walking experiments of the ant-type and carb-type tripod gaits, it is concluded that the $y$-direction force of the foot end of each leg can be neglected in solving the power consumption of robot mobile system, which realizes the dimensionality reduction about the statically indeterminate problem of robot. To maximize the support performance of each leg in the support phase, the necessary conditions of equal normal forces at the foot ends are analyzed under the tripod gait. The range of rotational angle is gained for the abductor joint of each leg in the support phase. Finally, the simplified power consumption model of mobile system under the tripod gait is established for the electrically driven large-load-ratio six-legged robot.

(4) To provide the necessary variable constraints for the numerical solution of the power consumption of robot mobile system, the stable walking constraints of the electrically driven large-load-ratio six-legged robot are analyzed. Then, the inequality constraint conditions, including the normal force of foot end, friction between foot and ground, and articulated driving, and the equality constraint conditions, involving the body height and equal foot force, are respectively acquired.

(5) According to the size and quality parameters of robot, results of simplification analysis, and constraint conditions of stable walking, the MATLAB software is employed to implement the numerical solution of the power consumption of robot mobile system under the variable working conditions of the tripod gait. The variable tendencies of the power consumption of robot mobile system are respectively gained with the changes in the rotational angles of the hip joint and knee joint, body height, and span. The articulated rotational zones and the 
ranges of body height and span are respectively determined under the lowest power consumption. Then, the body height should avoid $0.58 \mathrm{~m}$. The interval of span should be maintained at $0.18 \mathrm{~m}$ to $0.55 \mathrm{~m}$.

(6) Based on the developed prototype of the electrically driven large-load-ratio six-legged robot, the walking experiments of prototype are respectively actualized under the mixture-type II gait. The variable tendencies of the average power consumption of robot mobile system are acquired with changes in the duty ratio, body height and span. The values of the body height and span are also obtained under the minimum average power consumption of mobile system. The average power consumption curves of robot mobile system in the walking experiments are consistent with the power consumption curves of robot mobile system in the theoretical analysis, which further verifies the feasibility and correctness of the proposed analysis method about the power consumption of robot mobile system in this paper.

In view of the fact that the maximum walking speed of robot is not high and the walking speed is usually less than the maximum walking speed, the mathematical model of the power consumption of robot mobile system is established at the low walking speed for the electrically driven large-load-ratio six-legged robot. Then, it is difficult to directly solve the mathematical model of the power consumption of robot mobile system due to many of variables. Hence, the simplification of the mathematical model is facilitated for analyzing the power consumption of robot mobile system. This paper theoretically analyzes the power consumption characteristics of robot mobile system under the tripod gait on the hard flat ground. It verifies the rationality and accuracy of the theoretical analysis method of power consumption through the prototype walking experiments of the electrically driven large-load-ratio six-legged robot. Then, the theoretical and experimental research of the power consumption will be actualized for the mobile system of the electrically driven large-load-ratio six-legged robot in the rough terrain. The comparative analysis of the power consumption will be carried out between the hard flat ground and the rough terrain.

\section{Acknowledgements}

The authors sincerely thanks to Professor Liang Ding of Harbin Institute of Technology for his critical discussion and reading during manuscript preparation. The author would also like to thank the referees for their careful reading of the paper and helpful suggestions.

\section{Authors' contributions}

$\mathrm{H}-\mathrm{CZ}$ and NW were in charge of the whole trial; $\mathrm{H}-\mathrm{CZ}$ wrote the manuscript; H-CZ, H-BG, and Z-QD assisted with sampling and laboratory analyses. All authors read and approved the final manuscript.

\section{Authors' information}

Hong-Chao Zhuang, born in 1982, is currently an associate professor and a supervisor for master candidates at School of Mechanical Engineering, Tianjin University of Technology and Education, China. He received his $\mathrm{PhD}$ degree from Harbin Institute of Technology, China, in 2015. His research interests include special robot systems and intelligent robotics.

Ning Wang, born in 1981, is currently a lecturer at School of Information Technology Engineering, Tianjin University of Technology and Education, China. She received her $\mathrm{PhD}$ degree from Yanshan University, China, in 2015. Her research interests include special robot systems and control of legged robots.

Hai-Bo Gao, born in 1970, is currently a professor and a supervisor for $\mathrm{PhD}$ candidates at State Key Laboratory of Robotics and System, Harbin Institute of Technology, China. He received his $\mathrm{PhD}$ degree from Harbin Institute of Technology, China, in 2004. His research interests include special robot systems and aerospace mechanisms and control.

Zong-Quan Deng, born in 1956, is currently an Academician of Chinese Academy of Engineering, a professor, and a supervisor for $\mathrm{PhD}$ candidates at Harbin Institute of Technology, China. He received his master's degree from Harbin Institute of Technology, China, in 1984. His research interests include planetary rover technology and aerospace mechanisms and control.

\section{Competing interests}

The authors declare no competing financial interests.

\section{Funding}

Supported by National Natural Science Foundation of China (Grant No. 51505335) and Doctor Startup Projects of TUTE (Grant No. KYQD 1806).

\section{Author details}

${ }^{1}$ School of Mechanical Engineering, Tianjin University of Technology and Education, Tianjin 300222, China. ${ }^{2}$ School of Information Technology Engineering, Tianjin University of Technology and Education, Tianjin 300222, China. ${ }^{3}$ State Key Laboratory of Robotics and System, Harbin Institute of Technology, Harbin 150001, China. 


\section{Availability of data and materials}

The datasets supporting the conclusions of this article are included within the article.

\section{Consent for publication}

Not applicable

\section{Ethics approval and consent to participate}

Not applicable

\section{References}

[1] K Nozaki, T Murakami. A motion control of two-wheels driven mobile manipulator for human-robot cooperative transportation. Proceedings of the 35th Annual Conference of the IEEE Industrial Electronics Society, IECON 2009, Porto, Portugal, November 3-5, 2009: 1574-1579.

[2] B L Luk, D S Cooke, S Galt, et al. Intelligent legged climbing service robot for remote maintenance applications in hazardous environments. Robotics and Autonomous Systems, 2005, 53(2): 142-152.

[3] B H Wilcox, T E Litwin, J J Biesiadecki, et al. ATHLETE: A cargo handling and manipulation robot for the moon. Journal of Field Robotics, 2007, 24(5): 421-434.

[4] T Tsumaki, H Kobayashi, E Nakano, et al. Development of a practically scaled walking robot for steep terrain of forestry ground. Journal of the Robotics Society of Japan, 2009, 27(4): 470-480.

[5] D Wooden, M Malchano, K Blankespoor, et al. Autonomous Navigation for BigDog. Proceedings of the IEEE International Conference on Robotics and Automation, Anchorage, Alaska, USA, May 3-7, 2010: 4736-4741.

[6] E P Krotkov, R G Simmons, W L Whittaker. Ambler: Performance of a six-legged planetary rover. Acra Asrronaurrca, 1995, 35(1): 75-81.

[7] V Sunspiral, D W Wheeler, D Chavez-Clemente, et al. Development and field testing of the footfall planning system for the ATHLETE robots. Journal of Field Robotics, 2012, 29(3): 483-505.

[8] J E Bares, D S Wettergreen. Dante II: Technical description, results, and lessons learned. The International Journal of Robotics Research, 1999, 18(7): 621-649.

[9] H C Zhuang, H B Gao, Z Q Deng, et al. A review of heavy-duty legged robots. Science China Technological Sciences, 2014, 57(2): 298-314.

[10] H C Zhuang, N Wang, H B Gao, et al. Quickly obtaining range of articulated rotating speed for electrically driven large load-ratio six-legged robot based on maximum walking speed method. IEEE Access, 2019, 7(1): 29453-29470.

[11] H C Zhuang, N Wang, H B Gao, et al. Autonomous fault-tolerant gait planning research for electrically driven large-load-ratio six-legged robot. Proceedings of the 12th International Conference on Intelligent Robotics and Applications, Shenyang, China, August 8-11, 2019: 231-244.

[12] M F Silva, J A T Machado. A literature review on the optimization of legged robots. Journal of Vibration and Control, 2011, 18(12): 1753-1767.

[13] M F Silva, J A T Machado. A historical perspective of legged robots. Journal of Vibration and Control, 2007, 13(9-10): 1447-1486.

[14] M F Silva, J A T Machado. Kinematic and dynamic performance analysis of artificial legged systems. Robotica, 2008, 26(1): 19-39.
[15] D Sanz-Merodio, E Garcia, P Gonzalez-de-Santos. Analyzing energy-efficient configurations in hexapod robots for demining applications. Industrial Robot, 2012, 39(4): 357-364.

[16] S S Roy, D K Pratihar, Soft computing-based expert systems to predict energy consumption and stability margin in turning gaits of six-legged robots. Expert Systems with Applications, 2012, 39: 5460-5469.

[17] K Inoue, $T$ Tsurutani, $T$ Takubo, et al. Omni-directional gait of limb mechanism robot hanging from grid-like structure. Proceedings of the IEEE/RSJ International Conference on Intelligent Robots and Systems, Beijing, China, October 9-15, 2006: 1732-1737.

[18] K Hauser, T Bretl, J C Latombe, et al. Motion planning for legged robots on varied terrain. International Journal of Robotics Research, 2008, 27(11-12): 1325-1349.

[19] S S Roy, D K Pratihar. Effects of turning gait parameters on energy consumption and stability of a six-legged walking robot. Robotics and Autonomous Systems, 2012, 60: 72-82.

[20] K Yoneda, K Suzuki, Y Kanayama, et al. Gait and foot trajectory planning for versatile motions of a six-legged robot. Journal of Robotic Systems, 1997, 14(2): 121-133.

[21] H K Kim, D Won, O Kwon, et al. Foot trajectory generation of hydraulic quadruped robots on uneven terrain. Proceedings of the 17th IFAC, Seoul, Korea, July 6-11, 2008: 3021-3026.

[22] J T Lei, F Wang, H Y Yu, et al. Energy efficiency analysis of quadruped robot with trot gait and combined cycloid foot trajectory. Chinese Journal of Mechanical Engineering, 2014: 27(1): 138-145.

[23] H C Zhuang, H B Gao, L Ding, et al. Method for analyzing articulated torques of heavy-duty six-legged robot. Chinese Journal of Mechanical Engineering, 2013, 26(4): 801-812.

[24] H C Zhuang, H B Gao, Z Q Deng, et al. Method for analyzing articulated rotating speeds of heavy-duty six-legged robot. Journal of Mechanical Engineering, 2013, 49(23): 44-52. (in Chinese)

[25] H C Zhuang, H B Gao, Z Q Deng. Analysis method of articulated torque of heavy-duty six-legged robot under its quadrangular gait. Applied Sciences-Basel, 2016, 6(11), 323: 1-21.

[26] H C Zhuang, H B Gao, Z Q Deng. Gait planning research for an electrically driven large-load-ratio six-legged robot. Applied Sciences-Basel, 2017, 7(3), 296: 1-17.

[27] Z Liu, H C Zhuang, H B Gao, et al. Static force analysis of foot of electrically driven heavy-duty six-legged robot under tripod gait. Chinese Journal of Mechanical Engineering, 2018, 31(63): 1-15.

[28] B Vanderborght, B Verrelst, R V Ham, et al. Exploiting natural dynamics to reduce energy consumption by controlling the compliance of soft actuators. International Journal of Robotics Research, 2006, 25(4): 343-358.

[29] M Kaneko, S Tachi, K Tanie, et al. Basic study on similarity in walking machine from a point of energetic efficiency. IEEE Journal on Robotics and Automation, 1987, 3(1): 19-30.

[30] J Estremera, K J Waldron. Thrust control, stabilization and energetics of a quadruped running robot. International Journal of Robotics Research, 2008, 27(10): 1135-1151.

[31] T Zielinska. Walking machines for exploration-optimizing the energy spendings. Proceedings of the 10th International Workshop on Robot Motion and Control, Poznan, Poland, July 7-8, 2015: 6-8.

[32] $\mathrm{T}$ Zielinska. Minimizing energy cost in multi-legged walking machines. Journal of Intelligent and Robotic Systems, 2017, 85(3-4): 431-447.

[33] B S Lin, S M Song. Dynamic modeling, stability, and energy efficiency of a quadrupedal walking machine. Journal of Robotic Systems, 2001, 18(11): 657-670. 
[34] M F Silva, J A Tenreiro Machado, A M Lopes. Energy analysis of multi-legged locomotion systems. Proceedings of the 4th International Conference on Climbing and Walking Robots, Karlsruhe, Germany, September 24-26, 2001: 143-150.

[35] B Jin, C Chen, W Li. Power consumption optimization for a hexapod walking robot. Journal of Intelligent and Robotic Systems, 2013, 71: 195-209.

[36] D W Marhefka, D E Orin. Gait planning for energy efficiency in walking machines. Proceedings of the IEEE International
Conference on Robotics and Automation, Albuquerque, NM, USA, April 25-25, 1997: 474-480.

[37] J Nishii. Legged insects select the optimal locomotor pattern based on the energetic cost. Biological Cybernetics, 2000, 83(5): 435-442.

[38] P Gonzalez de Santos, E Garcia, R Ponticelli, et al. Minimizing energy consumption in hexapod robots. Advanced Robotics, 2009, 23 : 681-704. 